\title{
A Long Look at the Be/X-Ray Binaries of the Small Magellanic Cloud
}

\author{
J.L. Galache \\ Harvard-Smithsonian Center for Astrophysics, 60 Garden Street, Cambridge, MA 02138 \\ R.H.D. Corbet ${ }^{1}$ \\ NASA Goddard Space Flight Center, Greenbelt, MD 20771 \\ M.J. Coe \\ School of Physics and Astronomy, University of Southampton, Southampton SO17 1BJ, UK \\ S. Laycock \\ Harvard-Smithsonian Center for Astrophysics, 60 Garden Street, Cambridge, MA 02138 \\ M.P.E. Schurch \\ School of Physics and Astronomy, University of Southampton, Southampton SO17 1BJ, UK \\ C. Markwardt ${ }^{2}$ \\ NASA Goddard Space Flight Center, Greenbelt, MD 20771 \\ F.E. Marshall \\ NASA Goddard Space Flight Center, Greenbelt, MD 20771 \\ and \\ J. Lochner ${ }^{3}$ \\ NASA Goddard Space Flight Center, Greenbelt, MD 20771
}

\begin{abstract}
${ }^{1}$ University of Maryland, Baltimore County, MD 21250

${ }^{2}$ Department of Astronomy, University of Maryland, College Park, MD 20742

${ }^{3}$ Universities Space Research Association
\end{abstract}


We have monitored $41 \mathrm{Be} / \mathrm{X}$-ray binary systems in the Small Magellanic Cloud over $\sim 9$ years using PCA-RXTE data from a weekly survey program. The resulting light curves were analysed in search of orbital modulations with the result that 10 known orbital ephemerides were confirmed and refined, while 10 new ones where determined. A large number of X-ray orbital profiles are presented for the first time, showing similar characteristics over a wide range of orbital periods. Lastly, three pulsars: SXP46.4, SXP89.0 and SXP165 were found to be misidentifications of SXP46.6, SXP91.1 and SXP169, respectively.

Subject headings: galaxies: individual (Small Magellanic Cloud) - pulsars: general - X-rays: binaries

\section{Introduction}

The Small Magellanic Cloud (SMC) has become a fertile orchard of High-Mass Xray Binaries (HMXBs), with 49 confirmed systems (Coe et al. 2005; McGowan et al. 2007). Given that, from extrapolation of the Milky Way's population (and even correcting for the higher Be/B ratio in the SMC (Maeder et al. 1999)), one would expect to find only 3-4 systems, it is clear that the SMC is a special place where recent star formation has provided an abundance of HMXBs; indeed, the SFR/M (Star Formation Rate/Mass) of the SMC is 150 times that of the Milky Way (Grimm et al. 2003). Of particular significance is the fact that only one of these binary systems is not a Be/X-ray binary (SMC X-1 is the sole supergiant system discovered so far). This large number of Be/X-ray binary systems, conveniently located within the $3^{\circ} \times 3^{\circ}$ area of the SMC, provides an unrivaled opportunity to study this population as a whole, as well as individually. With a $2^{\circ}$ FWZI field of view, high timing resolution $(1 \mu \mathrm{s})$, and sensitive enough to detect the $10^{36}-10^{38} \mathrm{erg} \mathrm{s}^{-1}$ luminosities typical of these systems when in outburst, the PCA instrument (Jahoda et al. 1996, 2005) on board $R X T E$ is well suited for a long-term monitoring survey of the SMC.

The different types of X-ray activity displayed by Be/X-ray transient systems were classified by Stella et al. (1986) into the following categories:

- Persistent low-luminosity X-ray emission $\left(L_{\mathrm{x}} \lesssim 10^{36} \mathrm{erg} \mathrm{s}^{-1}\right)$ or none detectable (in which case the system is said to be in quiescence).

- Type I outbursts: Outbursts of moderate intensity $\left(L_{\mathrm{x}} \simeq 10^{36}-10^{37} \mathrm{erg} \mathrm{s}^{-1}\right)$ and short duration (a few days) generally recurring with the orbital period of the system and taking place at, or close to the time of periastron passage. 
- Type II outbursts: Giant X-ray outbursts $\left(L_{\mathrm{x}} \gtrsim 10^{37} \mathrm{erg} \mathrm{s}^{-1}\right)$ lasting for weeks or months that generally show no correlation with orbital phase.

The data presented in this paper spans November 1997 - November 2006, and builds upon the work of Laycock et al. (2005), who analysed the first 4 years of data. Following is a brief description of the survey so far, which is still ongoing as of June 2007.

\subsection{The survey}

The initial observations of the SMC with RXTE began in 1997. The first observation took place in November of that year when an outburst detected by the ASM was missidentified as SMC X-3. It was RXTE's second year of operation, and only SMC X-1, 2 and 3, SXP2.76 and SXP8.88 were known. From these initial observations it soon became apparent that there were more than 5 X-ray pulsars in the SMC. The observations carried out within the next year brought about the discovery of 5 new systems: SXPs 46.6, 59.0, 74.7, 91.1 and 169. Other pulsars were also discovered or detected with EINSTEIN, ASCA, BeppoSAX and ROSAT.

1999 marked the beginning of a coordinated survey of the SMC using the PCA. The PCA's $2^{\circ}$ field of view provides coverage of a wide area of the SMC, which allows many pulsars to be monitored with just one pointing. A number of different pointing positions have been used throughout the years and are given in Table 1 (see also Fig. 1); some of the less observed ones never received a name and are omitted from the table. The most frequently observed is Position 1 (later renamed to A), which has been the main pointing position since AO4, except during AO6 and AO7, when Position 5 was the main target. In total, the collected data spans $\sim 9$ years.

The survey has gone through various phases characterised by different observing positions and/or observing modalities. Phases $1-4$ have already been described in Laycock et al. (2005) but they are outlined here again, together with the two latest phases not included in previous studies.

Phase 1 (AO2-AO3): These observations used Positions 1a, $1 \mathrm{~b}$ and $1 \mathrm{c}$ and are described in Lochner et al. (1999a, b) . Only 30 observations were carried out, their main purpose being to monitor the 5 newly discovered pulsars in those regions.

Phase 2 (AO4): Positions 1-4 were defined; Position 1 overlaps most of Positions 1a-c and contained the new pulsars, Positions 2 and 4 cover the rest of the bar of the SMC 
while Position 3 covers the area of the wing containing SMC X-1. A continued survey began at this point in time, with $\sim 3$ ks observations being made once a week, mostly of Position 1, with some occasional looks at the other positions.

Phase 3 (AO5-AO6): Only Position 5 was monitored, and was done weekly so as not to create gaps in the data; the majority of the most active systems located in Position 1 also fell within the field of view of Position 5. Time allotted for the project was increased to an average of $\sim 5 \mathrm{ks}$ per observation, thus providing better sensitivity to longer period pulsations.

Phase 4 (AO7-AO9): Weekly monitoring returned to Position 1, now renamed Position A, with additional observations of the other positions $(\mathrm{B}, \mathrm{C}$ and $\mathrm{D}$, which are very close to 2, 3 and 4, respectively) being made once a month. These monthly pointings were $\sim 15 \mathrm{ks}$, while the weekly ones were increased to $\sim 6-7 \mathrm{ks}$.

Phase 5 (AO10): The time available for the monthly observations of alternate positions was invested into increasing the length of the weekly observations of Position A to $\sim 10 \mathrm{ks}$, this being the only monitored position.

Phase 6 (AO11): Having mainly monitored the central bar of the SMC, it was decided to move to another location at the northeastern tip of the bar, near position B. This new location, Position X, was monitored for $\sim 10$ ks weekly for 18 weeks, but was abandoned due to lack of pulsar activity. Observations then changed to Position D.

\subsection{Data reduction}

Cleaning of the raw light curves was achieved via a pipeline employing various FTOOLS1 routines (Blackburn 1995). Initial filtering required the data to come from observations offset from the target no more than $0.004^{\circ}$, with an elevation above the Earth's horizon of more than $5^{\circ}$, and not taken during times of high background. Good Xenon mode data from the top anode layer only were used, within an energy range of $3-10 \mathrm{keV}$. Data were binned at $0.01 \mathrm{~s}$ intervals, while background files were generated in $16 \mathrm{~s}$ bins. For each time-step, the net flux was divided by the number of active PCUs to give the counts $\mathrm{PCU}^{-1} \mathrm{~s}^{-1}$. Then, the light curves time tags were corrected to the Solar System barycenter, removing timing variations caused by the satellite's motion around the Earth and Sun. Finally, short light

\footnotetext{
${ }^{1}$ http://heasarc.gsfc.nasa.gov/ftools/
} 


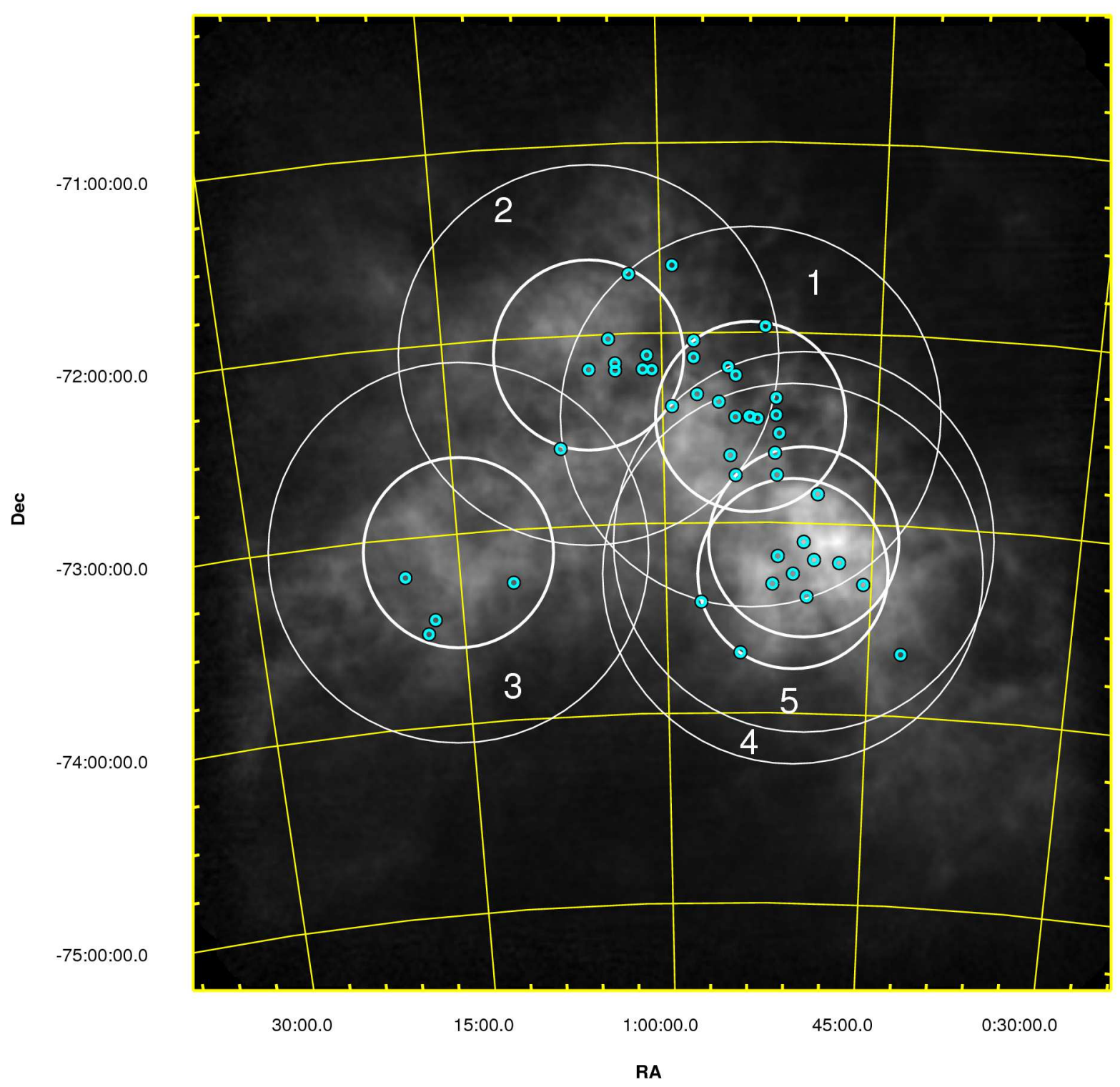

Fig. 1. - Map of the SMC H I distribution with the 5 main pointing positions of $R X T E$ during the survey shown as numbered white circles. For each pointing, the inner circle has a diameter of $1^{\circ}$, the outer of $2^{\circ}$. For clarity, Positions $\mathrm{A}, \mathrm{B}, \mathrm{C}, \mathrm{D}$ and $\mathrm{X}$ have not been plotted, as they are very close to Positions 1, 2, 3, 4 and 2, respectively. Pulsars with known positions are marked by small circles. H I data from Stanimirovic et al. (1999). 
curves belonging to the same observation (which had been split up due to SAA passage, Earth occultation or flares) were pieced together into a long light curve spanning the whole observation.

\subsection{Collimator correction}

The collimator of each PCU consists of a number of hexagonal cells joined in a honeycomb structure. The hexagonal tubes are not perfectly parallel with the result that all the PCUs are slightly off center. Hence, the resulting collimator response pattern has an elliptical hexagon shape with the maximum throughput being slightly off center.

In order to account for differences in observed flux from a pulsar when observed at different pointing positions, a collimator correction was applied a posteriori to each pulsar's count rate. A look-up table approach was used, where each pulsar had a collimator response calculated for each of the pointing positions used in the survey (see Table 1).

A pulsars with unknown position cannot be collimator corrected, so significant detections may not rise above the noise level in the long-term light curve unless the outbursts are very bright and/or the pulsar is located close to the center of the field of view. Type I outbursts may only appear bright when the pulsar is close to the center of the field of view (as with SXP59.0 in Position 1/A, Fig. 17), in which case the collimator correction is small anyway. To overcome this limitation pulsars with unknown positions had coordinates "guessed" for them on the basis of which observing positions they had been detected in throughout the mission; the coordinates assigned were approximately at the center of the region formed by the overlap of the observing positions in which it had been previously detected.

\subsection{Data analysis}

Once the cleaned light curve for an observation was obtained, it was run through a timing analysis package using the Lomb-Scargle method (Lomb 1976; Scargle 1982) and numerically implemented following the prescription by Press \& Rybicki (1989). Two different power spectra were generated for each light curve, each spanning different period spaces (from $P_{\text {min }}$ to $\left.P_{\max }\right)$, at different timing resolutions $(\Delta f)$, and pulsars were later searched for in their corresponding group according to their pulse period. The parameters used for each group are listed in Table 2, The reason for creating two groups was to obtain densely sampled periodograms at long periods without creating excessively large files. The parameters were chosen such that both groups would contain approximately the same number of independent 
frequencies, $M$.

Once the periodograms were calculated, it was necessary to establish an objective way to determine which peaks in the power spectra were real; this is covered in Appendix A.

\subsubsection{Pulsar Detection}

Pulsars were searched for within the data using an algorithm specifically created for the task. Each light curve's two power spectra were scanned to look for the pulsars in groups 1 and 2 ; the steps used were as follows:

Step 1: The highest peak (at a period $P$ ) above $90 \%$ global significance in the power spectrum is found and, either identified as a known pulsar, or flagged as an "unknown".

Step 2: The light curve is folded at period $P$ to produce a pulse profile.

Step 3: Using the pulse profile as a template, the pulsations are then subtracted from the light curve and a power spectrum of the cleaned light curve is produced.

Step 4: This power spectrum is subtracted from the previous one to create a pulsar-specific power spectrum (or $\mathrm{P}^{2} \mathrm{~S}^{2}$ ), which shows only the contribution of the individual pulsar to the power spectrum. This method allows one to see the possible harmonics that may have been lost in the noise or confused with the fundamental of another pulsar. The power of the fundamental and harmonic(s) peaks in the $\mathrm{P}^{2} \mathrm{~S}^{2}$ is measured and a pulse amplitude estimated using Eq. (A5). The significance of the detection is taken to be the local significance of a peak of power equal to that which the detected signal would have if the power in the harmonics were concentrated in the fundamental.

Step 5: Repeat previous steps until all signals with peaks above $90 \%$ significance have been removed.

Step 6: To account for the remaining, dim pulsars, a stretch of the power spectrum centered on each pulsar's nominal frequency, and with a total width which is different for each pulsar (and depends on the pulsar's past period history), is searched for a peak. If no peak is found, then the significance of the detection is set to $0 \%$ and the amplitude to that of the average power within the region. If there is a peak, then the significance of the detection is set to the local significance and the amplitude of the pulsar calculated from the power in the peak according to Eq. (A4). No harmonics are searched for in this case. 
Table 1. RXTE's SMC survey pointing positions

\begin{tabular}{lcc}
\hline \hline \multicolumn{1}{c}{ Name } & RA $\left(^{\circ}\right)$ & Dec $\left(^{\circ}\right)$ \\
\hline 1a & 005207.8 & -722543.3 \\
1b & 005104.0 & -721344.0 \\
1c (SMC X-3) & 005454.8 & -722640.9 \\
1/A & 005353.0 & -722642.0 \\
2 & 010500.0 & -720600.0 \\
3 & 011500.0 & -730600.0 \\
4 & 005044.6 & -731604.8 \\
5 & 005000.0 & -730600.0 \\
B & 010500.0 & -720621.6 \\
C & 011500.0 & -732459.8 \\
D & 005000.0 & -730600.0 \\
X & 010500.0 & -720600.0 \\
SMC X-2 & 005433.3 & -734104.2 \\
\hline
\end{tabular}

Table 2. Parameters used for the Lomb-Scargle periodogram

\begin{tabular}{lcc}
\hline \hline & Group 1 & Group 2 \\
\hline$P_{\min }(\mathrm{s})$ & 0.5 & 10 \\
$P_{\max }(\mathrm{s})$ & 1000 & 3000 \\
$\Delta f(\mathrm{~Hz})$ & $10^{-5}$ & $5 \times 10^{-7}$ \\
Search range $(\mathrm{s})$ & $0.5-40$ & $40-3000$ \\
\hline
\end{tabular}




\subsubsection{Light Curve Generation and $P_{\text {orb }}$ Calculations}

Once the amplitude of the pulsed flux for every pulsar in every observation had been measured, a long-term light curve was created for each system showing its X-ray activity over the course of the survey. It is important to note that these light curves do not show the absolute flux, but rather the pulsed flux. Without knowing the pulsar's pulse fraction for each observation it is impossible to convert the pulsed flux amplitude into an absolute flux value. These light curves were then searched for periodicities, as the X-ray emission could show orbital modulations. Again, the Lomb-Scargle technique was employed, searching for periods in the appropriate range for each pulsar. In cases where Type II outbursts contaminated the Lomb-Scargle periodogram, these were removed from the data. In general, all bright outbursts were initially removed and only added back into the light curve if they coincided with the calculated ephemerides and their inclusion increased the significance of the calculated orbitla period. It is important to note that the collimator response correction was only applied to the data points whose local significance upon detection was $\geq 99 \%$, anything below this detection threshold was considered noise, and as such was not collimator corrected. $\S 2$ shows the results of these analyses.

\subsubsection{Orbital Profile Generation}

Based on the ideas of de Jager et al. (1988) and Carstairs (1992), we used the PhaseIndependent Folding (PIF) technique to create the orbital profiles. The method is as follows: the folded light curve is obtained from $m$ sets of $n$-binned folded light curves. To begin with, the light curve is folded at the desired period and the time values converted to phase space (ranging from 0 to 1 ). This "raw" folded light curve is then binned into $n$ bins (of width $1 / n)$ in the standard way, with each bin starting at phase $a / n$ (with $a=0,1,2, \ldots n-1)$. This step is repeated again, but this time the bins start at phase $a / n+1 /(n \times m)$. In this way we create $m$ folded light curves from the same data, each consisting of $n$ bins, and only differing in the starting phase of these bins2. The general expression for the phase at which each bin begins is $a / n+m /(n \times m)$. Now each folded light curve is further divided into $l=n \times m$ sub-bins, such that in each light curve there will be $n$ groups of $m$ consecutive bins with the same flux value. The final folded light curve will have $l$ bins, each of which is the average of the bins from the $m$ sets of light curves. The error in each bin will be given by the standard error calculated from the $m$ values that were averaged for each $l$-bin. In the present work we have used $n \times m=10 \times 5$.

\footnotetext{
${ }^{2}$ We define $\phi=0$ at the time of the first data point in each observation.
} 
The PIF method provides an efficient way of generating folded light curves from poorly sampled data as their shape will not depend on the starting point at which they are folded and, although only every $m^{\text {th }}$ bin will be independent, spurious flux values within bins will be evened out while real features will remain. Thus, we obtain the benefits of both wide bins (sufficient counts in each bin for good statistics) and narrow bins (sensitivity to narrow features in the profile).

\section{Results}

Following are the results obtained from the light curves of the observed SMC X-ray pulsars. In the triple-panel plots (generally the (a) plots), the top panel shows the Xray activity of each pulsar through the amplitude of the pulsed flux, with each solid line representing one observation. When an orbital period has been found, the ephemerides for the dates of maximum flux are over plotted as dashed vertical lines; where the orbital period is less than 25 days, only every other line is plotted for clarity. The middle panel shows the period at which the pulsar was detected, with the horizontal dashed line denoting the center of the pulsar's search range (note that only significant detections have their periods plotted). Finally, the bottom panel displays the significance of the neutron star's pulsations for each observation, with the three horizontal dashed lines marking, from bottom to top, the levels of 99, 99.99 and $99.9999 \%$ local significance. We considered a pulsar had been detected when its local significance was $\geq 99 \%$. In the (b) plots we show the Lomb-Scargle periodogram of the pulsed flux light curve (top) and the light curve folded at the orbital period (bottom). When horizontal dashed lines are plotted on the periodogram, these represent different levels of significance, which are (from bottom to top) 99, 99.9, 99.99, and 99.999\%. The coordinates given for each system are the most accurate at time or writing. When a Be/X-ray system has a confirmed optical counterpart, the coordinates of the counterpart are used. When no optical counterpart is known, but the system has been detected by Chandra and/or XMM, then it is the X-ray coordinates that were given. For some of the pulsars without an exact position, the coordinates provided by scans with $R X T E$ are given; these are the least precise and have errors of up to several arc minutes.

\subsection{SXP0.92}

PSR J0045-7319

RA 004535 , dec -731902 
History: First discovered as a radio pulsar by Ables et al. (1987) with a period of $0.926499 \pm 0.000003 \mathrm{~s}$ using the Parkes $64 \mathrm{~m}$ radio telescope. Kaspi et al. (1993) observed Doppler shifts in the pulse period which where consistent with a $51 \mathrm{~d}$ binary orbit with a companion star having mass $>4 M_{\odot}$. Optical observations of the field revealed a $16^{\text {th }}$ magnitude, $11 M_{\odot}, \mathrm{B} 1$ main-sequence star, which is likely the companion (Bell 1994).

Survey Results: SXP0.92 received $\sim 2$ years of uninterrupted coverage during AO5 and AO6 (see Fig. 2), during which time it was only once detected above $99 \%$ local significance (MJD 52334). The power spectrum does not show any significant periods.

\subsection{SXP2.16}

XTE SMC2165 RA 011900 , dec -731227

History: Discovered during the course of this survey by Corbet et al. (2003a) at $2.1652 \pm 0.0001 \mathrm{~s}$.

Survey Results: Due to its position near SMC X-1 it has only been within the field of view on 3 occasions: MJD 51220, 51263 and 51310. It was on this last date that it was discovered (its only significant detection).

\subsection{SXP2.37}

\section{SMC X-2}

\section{RA 005434 , dec -734103}

History: SMC X-2 was discovered in $S A S-3$ observations of the SMC carried out by Clark et al. (1978). Further outbursts were also observed by HEAO 1 and ROSAT, but no pulsations were detected until it was observed in the present survey by $R X T E$ during a long outburst lasting from January through to May, 2000 (Corbet et al. 2001; Laycock 2002).

Survey Results: After the aforementioned outburst, SMC X-2 was only detected on 3 further occasions (MJD 51974, 52025 and 52228), but at a much lower flux of $F_{\mathrm{x}_{\mathrm{pul}}}<$ 1 counts $\mathrm{PCU}^{-1} \mathrm{~s}^{-1}$ (see Fig. 3). Lomb-Scargle analysis of the data following the major outburst shows no clear periods. 


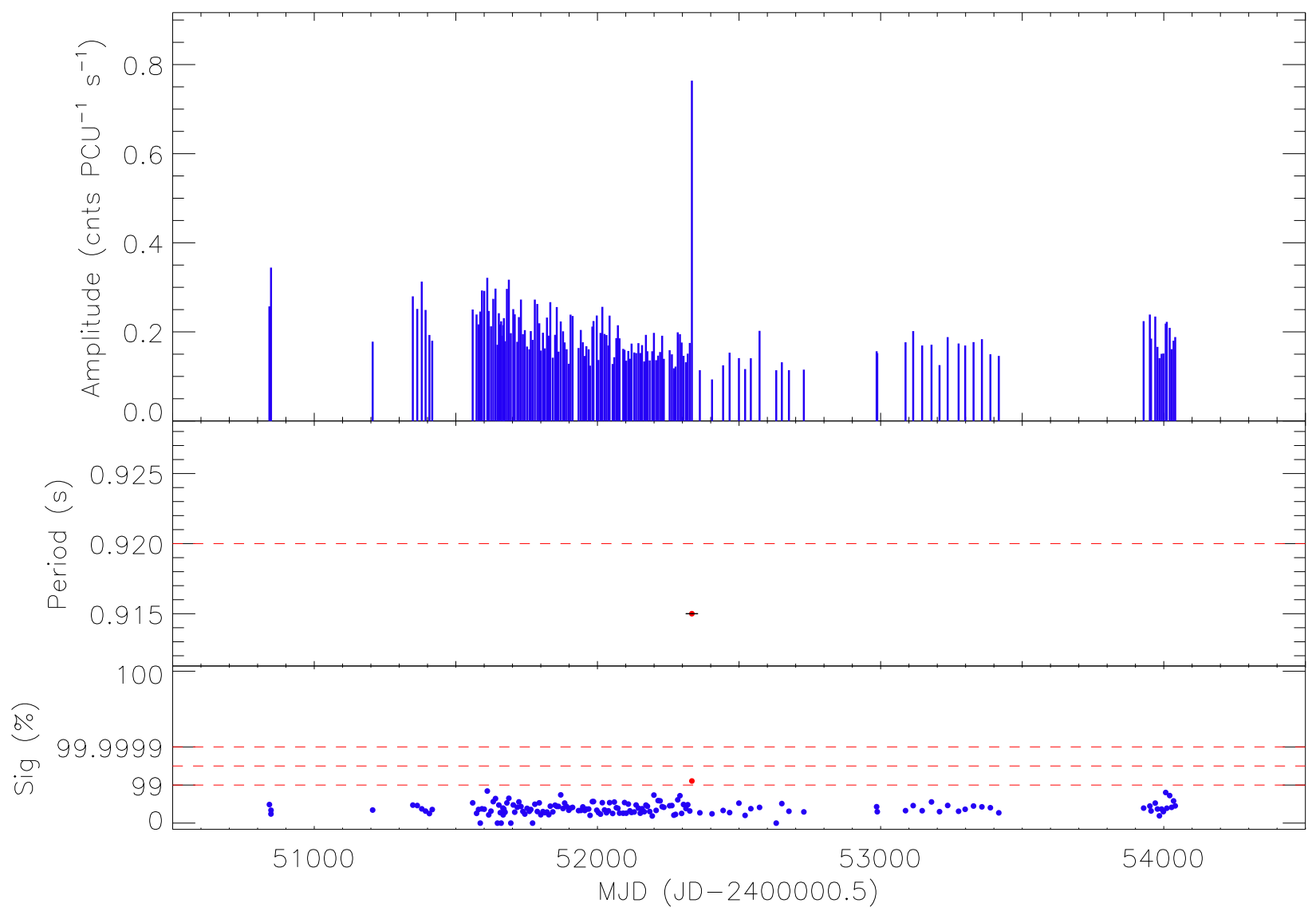

Fig. 2.- SXP0.92, X-ray amplitude light curve. 


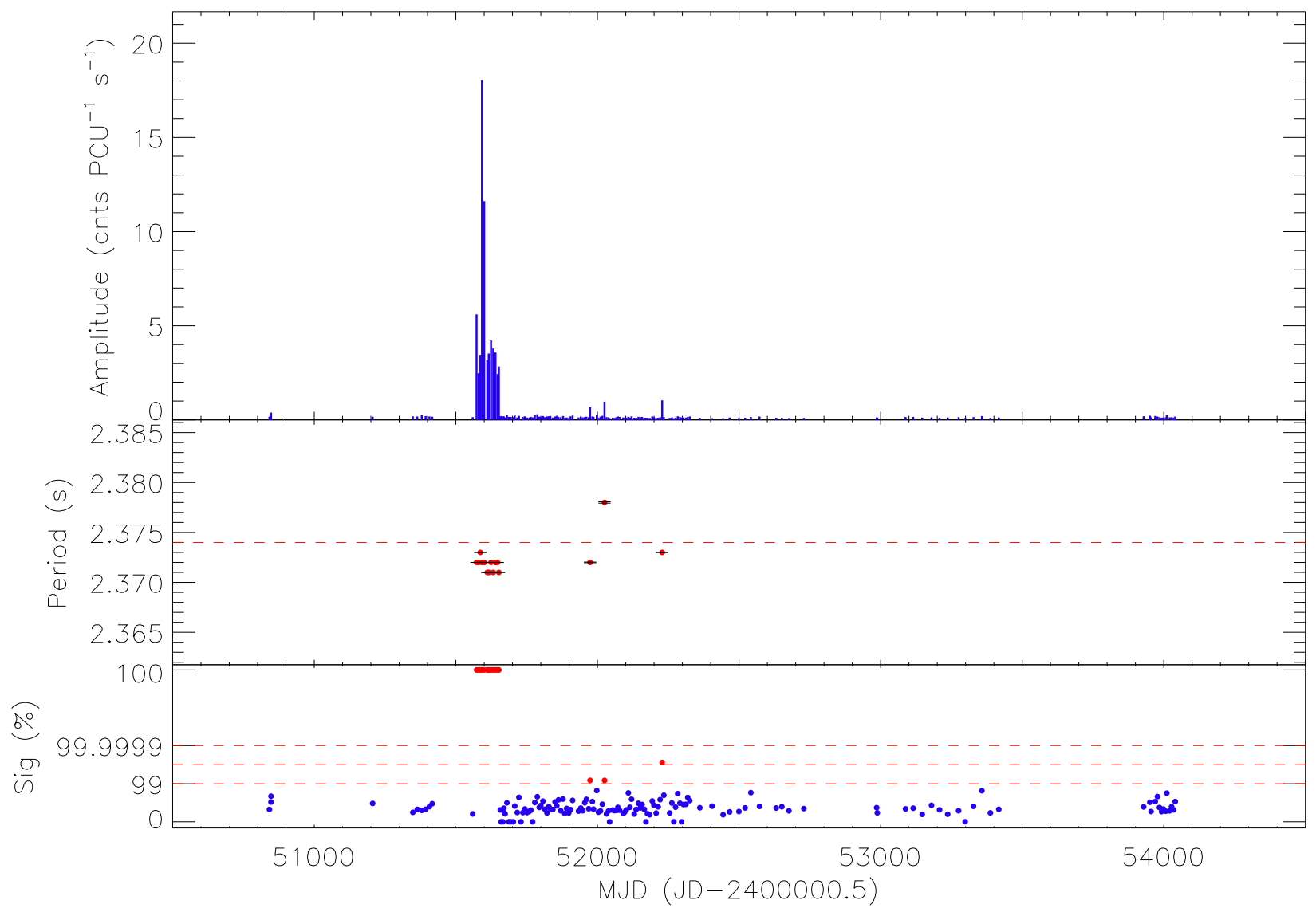

Fig. 3.- SXP2.37, X-ray amplitude light curve. 


\subsection{SXP2.76}

RX J0059.2-7138

\section{RA 0059 11.7, dec -713848}

History: First reported by Hughes (1994) from a ROSAT observation showing $2.7632 \mathrm{~s}$ pulsations that varied greatly with energy. In the low-energy band of the ROSAT PSPC $(0.07-0.4 \mathrm{keV})$ the source appears almost unpulsed while in the high-energy band $(1.0-$ $2.4 \mathrm{keV}$ ) the flux is $\sim 50 \%$ pulsed. Its optical counterpart was confirmed as a $14^{\text {th }}$ magnitude Be star by Southwell \& Charles (1996). Schmidtke et al. (2006) report a period of $82.1 \mathrm{~d}$ with a maximum at MJD 52188.9 from analysis of OGLE III data.

Survey Results: This source is near the edge of the field of view of Position 1/A so we would expect to detect only the brighter outbursts. Only 2 detections were made (MJD 52527 and 53327) and no orbital period can be extracted from the X-ray data (see Fig. 4). We find no power at the reported optical period and note that the two X-ray detections occured $\sim 10$ days after and $\sim 11$ days before the optical ephemeris's predicted maximum.

\subsection{SXP3.34}

AX J0105-722, RX J0105.3-7210

\section{RA 010502 , dec -721100}

History: Was reported as an ASCA source with $3.34300 \pm 0.00003$ s pulsations by Yokogawa \& Koyama (1998c). Its optical counterpart is proposed to be [MA93] 1506 (Coe et al. 2005), who also find an $11.09 \mathrm{~d}$ modulation in MACHO data. Although this could be the orbital period of the system (as it falls within the expected range on the Corbet diagram (Corbet 1984)), Schmidtke \& Cowley (2005a) report the find of a strong $1.099 \mathrm{~d}$ period in MACHO data and that the $11.09 \mathrm{~d}$ value is an alias of this main period. They attribute the modulation to non-radial pulsation in the Be star.

Survey Results: There have been no significant detections of the pulsar during this survey and timing analysis of the light curve reveals no clear periodicities.

\subsection{SXP4.78}

XTE J0052-723

RA 005206.6 , dec -722044 


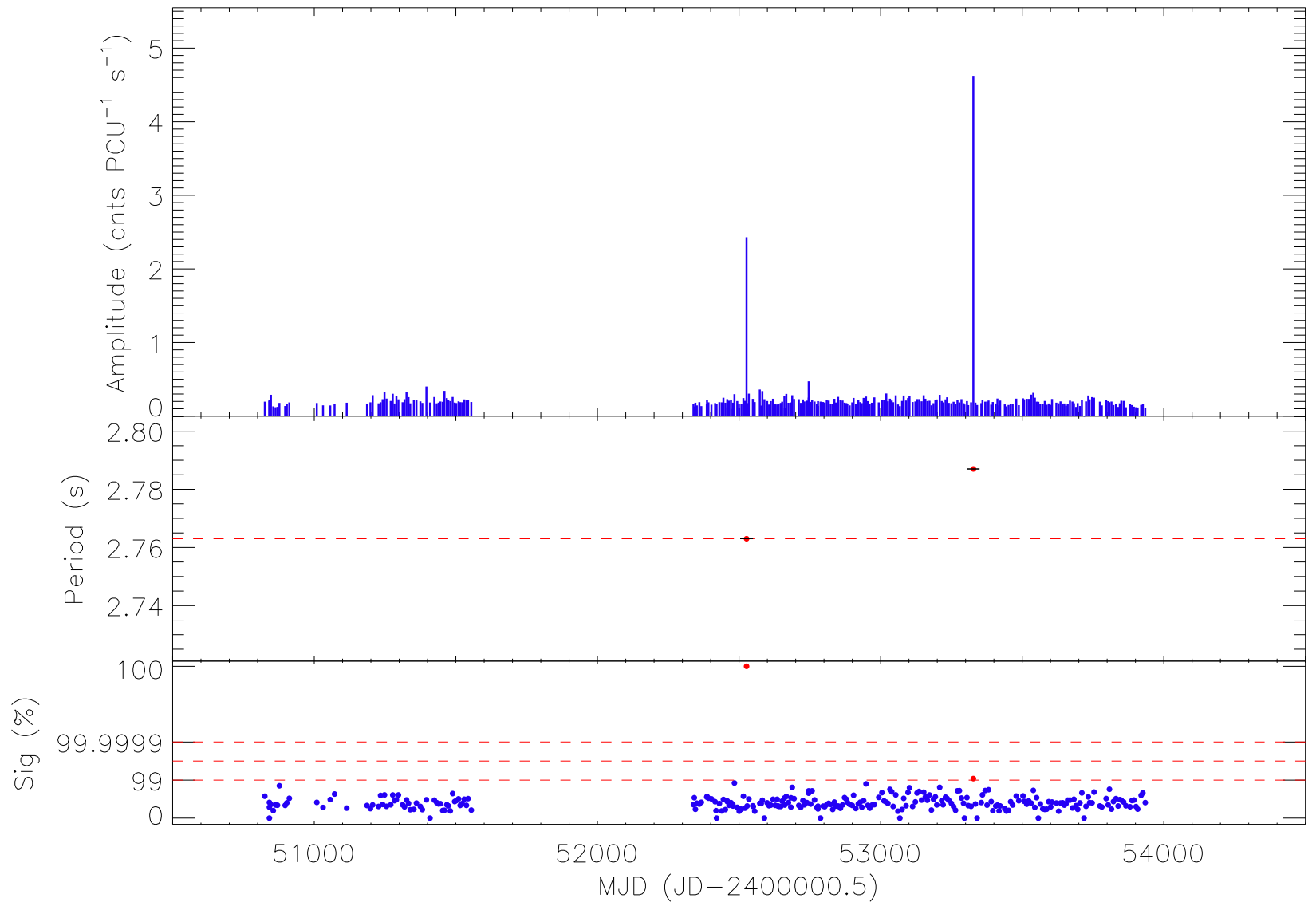

Fig. 4.- SXP2.76, X-ray amplitude light curve. 
History: Was discovered by the present survey in late December 2000 and reported in Laycock et al. (2003), where [MA93] 537 was proposed as the optical counterpart. Another possible counterpart is suggested in Coe et al. (2005) as the star AzV129 is found to have a $23.9 \pm 0.1 \mathrm{~d}$ period in both MACHO colours, which would agree with the expected orbital period inferred from the Corbet diagram.

Survey Results: SXP4.78 was detected on one occasion after its initial outburst (MJD 52729 ) at a much weaker $F_{\mathrm{x}_{\mathrm{pul}}} \simeq 0.8$ counts $\mathrm{PCU}^{-1} \mathrm{~s}^{-1}$. It then began a relatively long, bright (peaking at $\sim 1.2$ counts $\mathrm{PCU}^{-1} \mathrm{~s}^{-1}$ ) outburst on December 21, 2005 (MJD 53725) that lasted $\sim 7$ weeks (see Fig. 5). Despite the long outburst, no orbital modulation is apparent. Timing analysis using all the data finds no periods, while analysis of the data outside the two bright outbursts suggests a weak period at $34.1 \mathrm{~d}$

A 1 ks observation with Chandra was carried out on March $3^{\text {rd }} 2006$ in an attempt to establish the pulsar's coordinates. Unfortunately, the outburst had ended and no source was detected within the RXTE error box provided by Laycock et al. (2003).

\subsection{SXP6.85}

XTE J0103-728

RA 010253.1 , dec $=-724433$

History: First detected in April 2003 with a pulse period of $6.8482 \pm 0.0007 \mathrm{~s}$ (Corbet et al. $2003 \mathrm{~b})$. It was detected in outburst with $X M M$ on October $2^{\text {nd }} 2006$, which provided a more accurate position (Haberl et al. 2007). This allowed its optical counterpart to be identified as a Be star with $\mathrm{V}=14.59$, with a possible $24.82 \mathrm{~d}$ periodicity (Schmidtke \& Cowley 2007).

Survey Results: It has been detected on 3 other occasions (circa MJD 52885, 53440 and $53677)$ at varying fluxes $\left(F_{\mathrm{x}_{\mathrm{pul}}} \approx 0.8-1.8\right.$ counts $\left.\mathrm{PCU}^{-1} \mathrm{~s}^{-1}\right)$, but high above the noise level (see Fig. 6). Lomb-Scargle analysis of the data outside the 4 bright detections shows no significant period. When including these outbursts, we find a significant period of $112.5 \mathrm{~d}$; this is the minimum time lapse between any two outbursts and probably drives the result. Given its pulse period, and based on the spin-orbit relation, we do not discard the possibility that the real orbital period be $\frac{1}{2}$ or $\frac{1}{3}$ of this value. The current X-ray ephemeris is MJD $52318.5 \pm 7.9+\mathrm{n} \times 112.5 \pm 0.5 \mathrm{~d}$. We note that the outburst detected on October $2^{\text {nd }} 2006$ (MJD 54010) is consistent with the proposed ephemeris. 


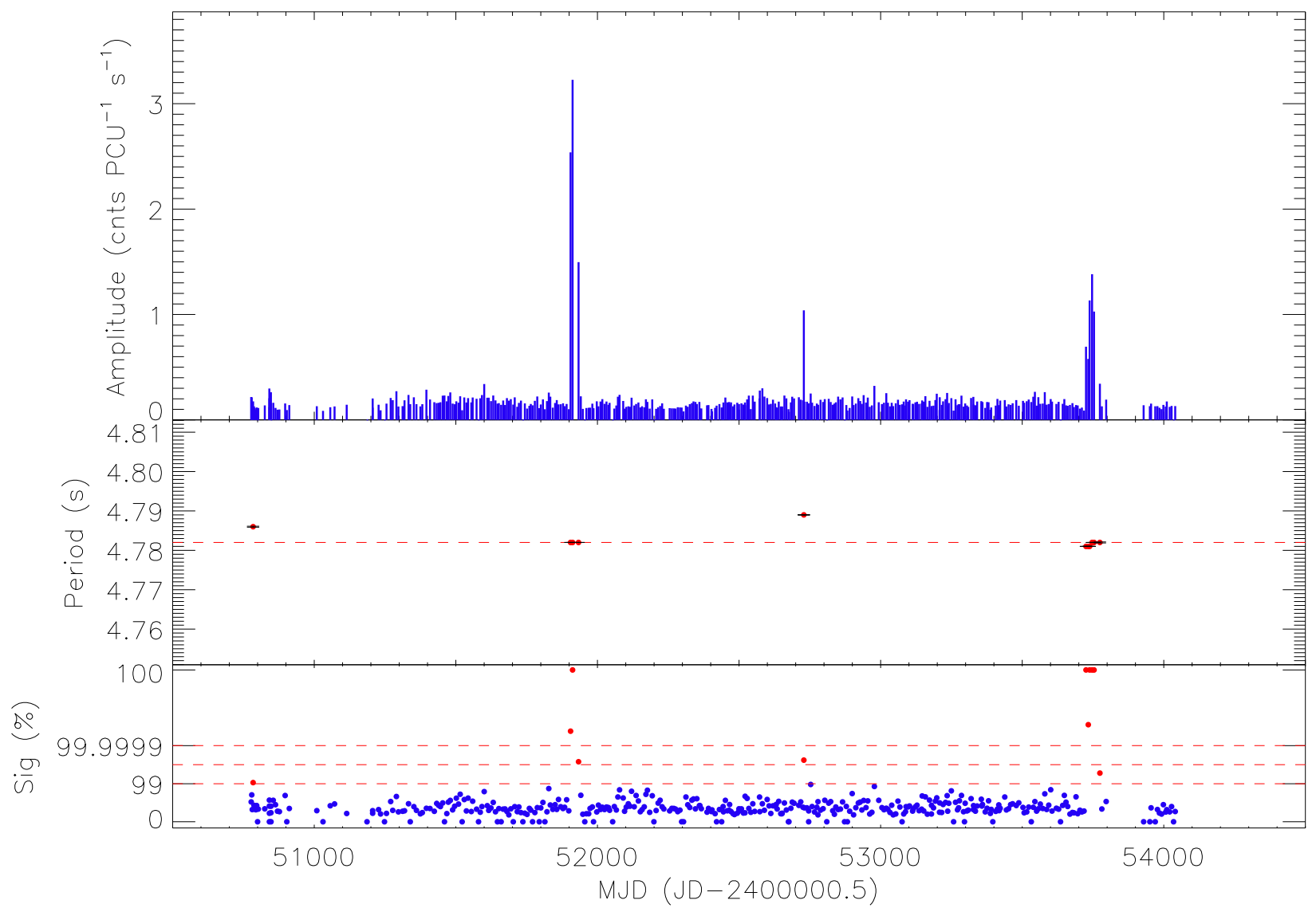

Fig. 5.- SXP4.78, X-ray amplitude light curve. 

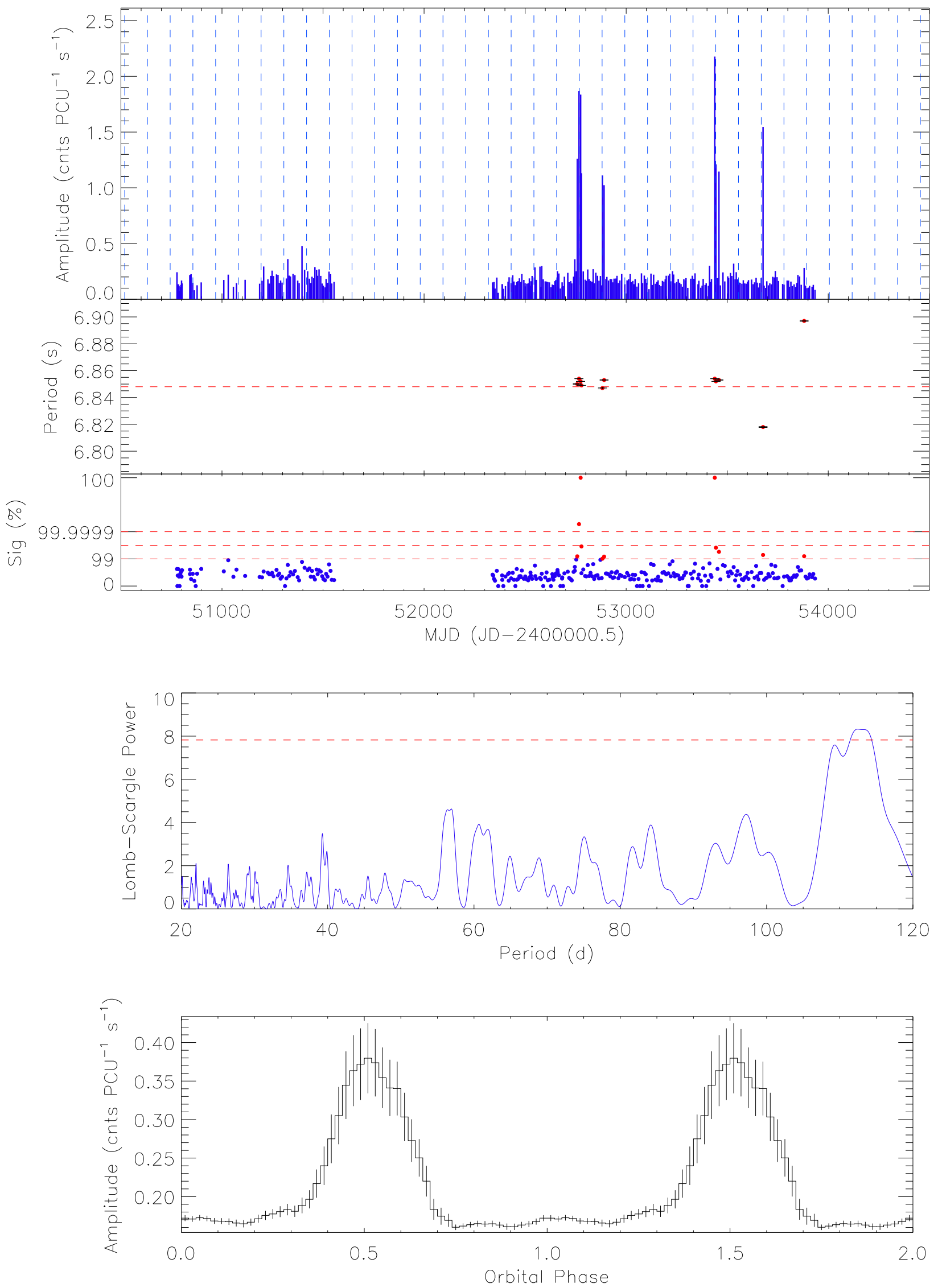

Fig. 6. - SXP6.85. a) Top: X-ray amplitude light curve. b) Middle: Lomb-Scargle power 


\subsection{SXP7.78}

\section{SMC X-3}

\section{RA 005205.8 , dec -722603.2}

History: Originally detected by Clark et al. (1978), it was not identified with the previously detected RXTE 7.78 s source until 2004 (Edge et al. 2004a). Corbet et al. (2003c, 2004b) proposed an orbital period from a series of recurrent X-ray outbursts (in the present survey) of $45.1 \pm 0.4 \mathrm{~d}$. An optical modulation in MACHO data was reported by Cowley \& Schmidtke (2004) (44.86 d) and Coe et al. (2005) (44.6 $\pm 0.2 \mathrm{~d})$; Edge (2005d) also found a strong $44.8 \pm 0.2 \mathrm{~d}$ modulation in the OGLE counterpart, present even when there was no significant $\mathrm{X}$-ray activity.

Survey Results: It has displayed in the past 10 years 3 distinct periods of outbursts $\left(F_{\mathrm{x}_{\mathrm{pul}}} \approx\right.$ 0.3 counts $\mathrm{PCU}^{-1} \mathrm{~s}^{-1}$ ), lasting $\sim 200-400 \mathrm{~d}$ (see Fig. 7(a)). Timing analysis of the complete light curve reveals a clear period at $44.92 \mathrm{~d}$; the ephemeris we derive is MJD $52250.9 \pm 1.4$ $+\mathrm{n} \times 44.92 \pm 0.06 \mathrm{~d}$. The folded light curve may show evidence of detections at apastron. During the longer of the outburst episodes (circa MJD 52500) the pulsar shows some spin up, about $\dot{P}=3.7 \times 10^{-10} \mathrm{~s} \mathrm{~s}^{-1}$, implying $L_{\mathrm{x}} \geq 2.3 \times 10^{37} \mathrm{erg} \mathrm{s}^{-1}\left(B \leq 3.6 \times 10^{12} \mathrm{G}\right)$. This pulsar is unique in that, despite the spin up observed during each of the individual outburst episodes, the overall spin evolution seems to show a long-term spin down.

\subsection{SXP8.02}

\section{CXOU J010042.8-721132}

\section{RA 010041.8 , dec -721136}

History: Proposed as the first anomalous X-ray pulsar (AXP) in the SMC by Lamb et al. (2002), they found the source to have displayed little variability in the past 20 years. It is characterised by a very soft spectrum and low luminosity $\left(\sim 1.5 \times 10^{35} \mathrm{erg} \mathrm{s}^{-1}\right)$.

Survey Results: During AO5 and AO6 it was outside the field of view of our observations. Coverage from AO7 onwards has been good and timing analysis of these dates shows a possible periodicity at $\sim 23.2 \mathrm{~d}$, which is likely driven by the only two clear detections, separated by that time range (see Fig. 8). This period disappears when removing these two detections from the data. If SXP8.02 is truly an anomalous pulsar, it is not expected to show periodicity in its X-ray emission. The two significant detections were observed at around $L_{\mathrm{x}}$ $=5.0 \times 10^{36} \mathrm{erg} \mathrm{s}^{-1}$ (unabsorbed, assuming a $50 \%$ pulse fraction and a power law spectrum of $\gamma=1)$. 

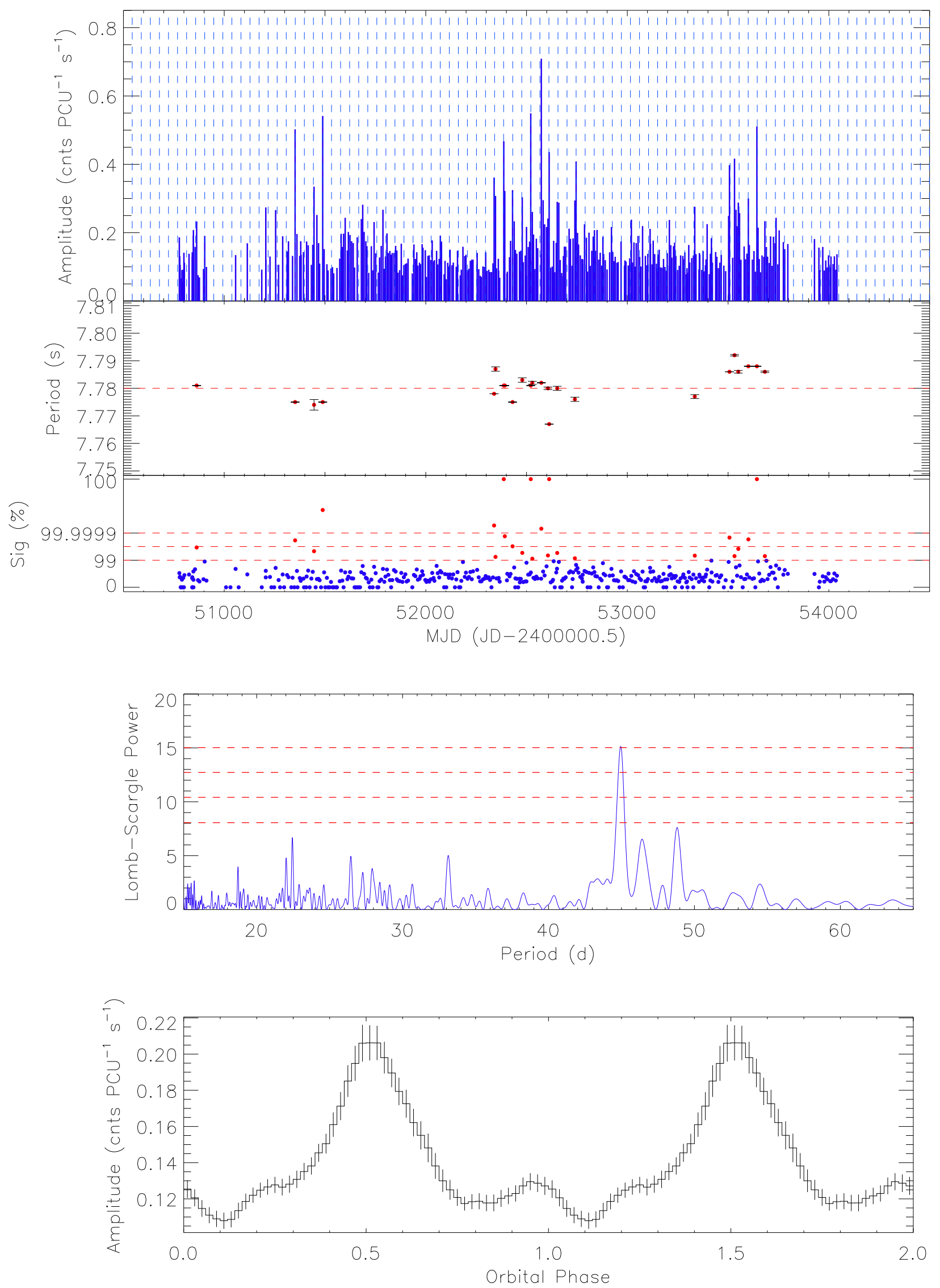

Fig. 7.- SXP7.78. a) Top: X-ray amplitude light curve. b) Middle: Lomb-Scargle power 


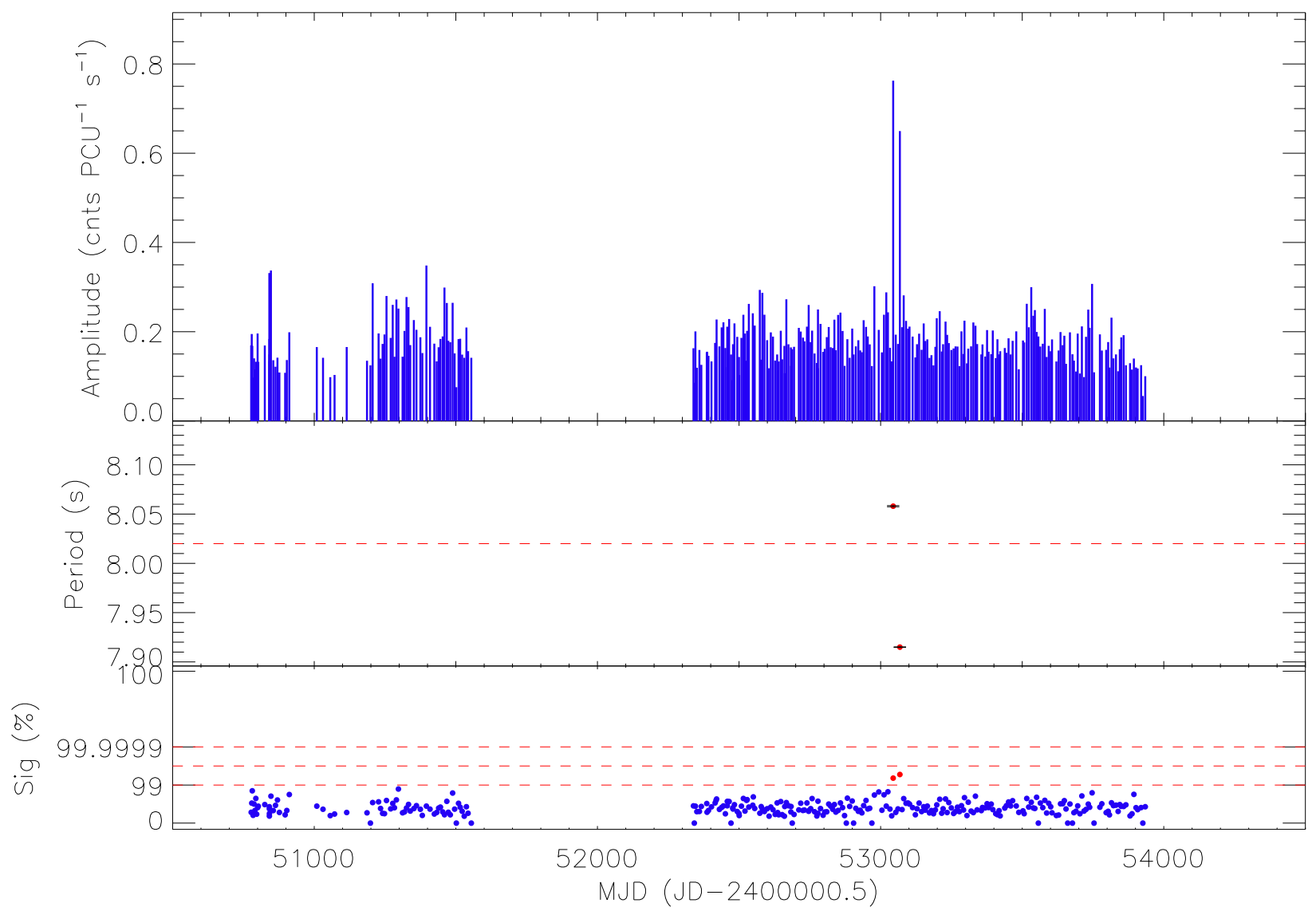

Fig. 8.- SXP8.02, X-ray amplitude light curve. 


\subsection{SXP8.88}

RX J0051.8-7231, 1E0050.1-7247, 1WGA J0051.8-7231

\section{RA 005152.0, dec -723151.7}

History: Israel et al. (1997) detected this source several times between 1979 and 1993. A number of optical counterparts have been proposed, and Haberl \& Sasaki (2000) believe [MA93] 506 to be the correct one. From a number of outbursts during July 2003-May 2004 Corbet et al. (2004a) derived an orbital ephemeris of MJD $52850 \pm 2+\mathrm{n} \times 28.0 \pm 0.3 \mathrm{~d}$. Schmidtke \& Cowley (2006) find an optical period of 33.4d in OGLE and MACHO data. Note: This system was incorrectly named SXP8.80 in Coe et al. (2005).

Survey Results: Two Type II outbursts together with the aforementioned series of Type I outbursts have been detected (see Fig. 9). A period of $\sim 28 \mathrm{~d}$ is found using Lomb-Scargle analysis on the data not containing the Type II outbursts, similar to the value from Corbet et al. (2004a). The new ephemeris from the survey data is MJD $52392.2 \pm 0.9+\mathrm{n} \times 28.47 \pm 0.04 \mathrm{~d}$. We note that the two Type II outbursts began roughly at the times of expected maximum flux, lasted about 1 orbit, and peaked $0.5 P_{\text {orb }}$ later. This could be explained by a number of scenarios: a) The neutron star forms an accretion disc around periastron, which it consumes throughout the orbit; b) an accretion disc is formed around periastron which is accreted onto the neutron star along the orbit at the same time as wind accretion (which would peak around apastron) is taking place; c) the Be star ejects matter forcefully enough that it moves outwards as an annulus and the neutron star is able to capture material along its orbit as it moves away from the Be star, but capture decreases as soon as it passes apastron.

None of these scenarios completely explain the behaviour of the X-ray emission during the Type II outbursts. If a) is correct, then why would accretion become greatest at apastron on both occasions. Scenario c) suffers a similar drawback as it would require the annulus to move at similar speeds on both outbursts in order for the outburst to peak precisely at apastron. Scenario b) would require a very dense wind in order for its accretion to be comparable to disc accretion. Simultaneous observations of this system in the X-ray and the optical during an outburst would help clarify this behaviour.

\subsection{SXP9.13}

AX J0049-732, RX J0049.2-7311

RA 004913.6 , dec -731139

History: Discovered during an ASCA observation in November 1997 (MJD 50765), pulsa- 

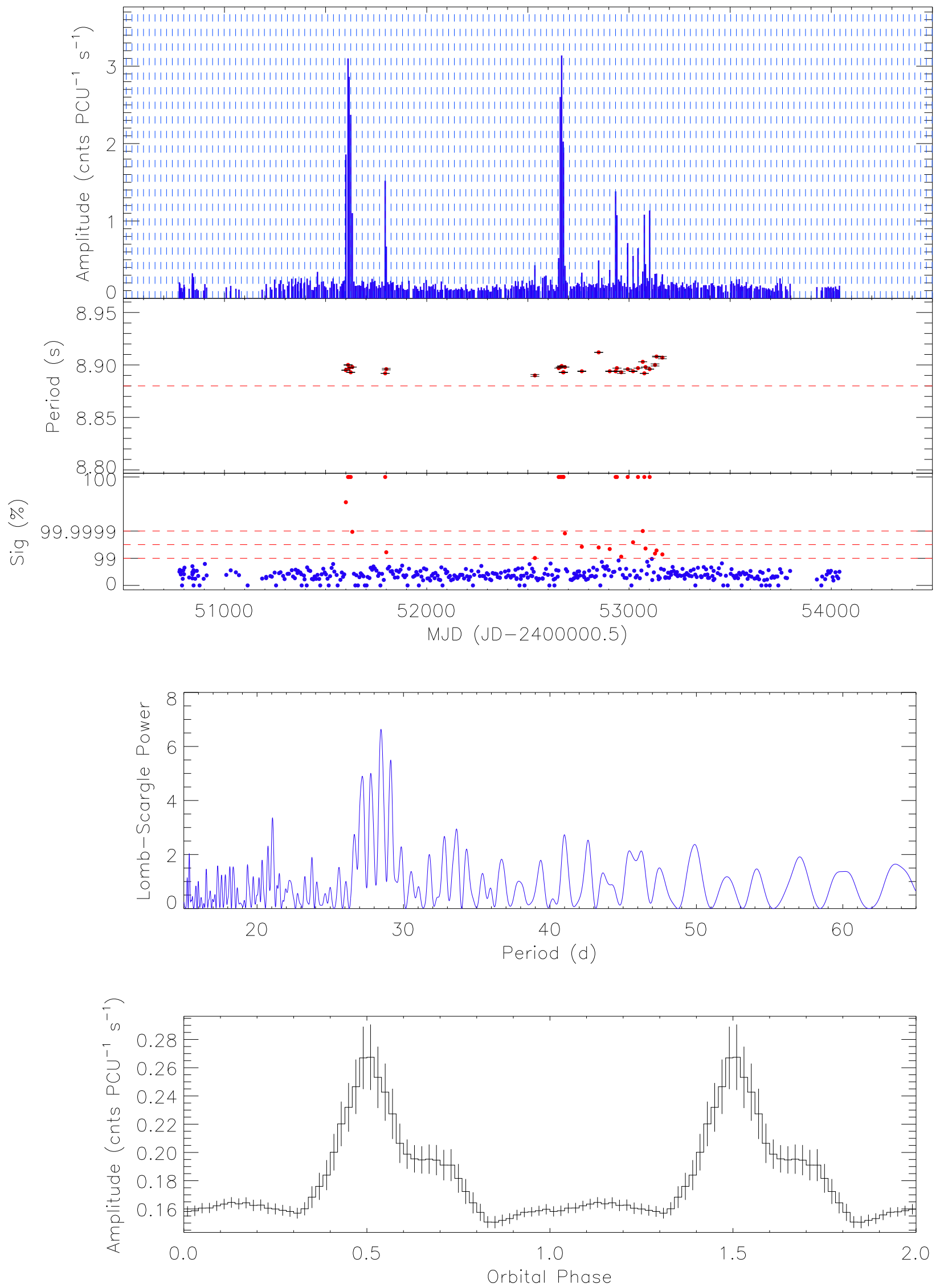

Fig. 9.- SXP8.88. a) Top: X-ray amplitude light curve. b) Middle: Lomb-Scargle power 
tions were detected at $9.1321 \pm 0.0004 \mathrm{~s}$ and $L_{\mathrm{x}}=3.3 \times 10^{35} \mathrm{erg} \mathrm{s}^{-1}$ (Imanishi et al. 1998). There has been much debate as to which is the correct optical counterpart to this source, Schmidtke et al. (2004) and Filipović et al. (2000) identify it with the ROSAT source RX J0049.5-7310 but Coe et al. (2005) conclude that it is a $\mathrm{H} \alpha$ source coincident with $\mathrm{RX}$ J0049.2-7311. Timing analysis of the OGLE data shows a peak at $40.17 \mathrm{~d}$ and its probable harmonic at $20.08 \mathrm{~d}$ (Edge 2005d). It should be noted that Schmidtke et al. (2004) find a $91.5 \mathrm{~d}$ or possibly an $\sim 187 \mathrm{~d}$ period for RX J0049.5-7310. It was detected by $A S C A$ on one further occasion on MJD 51645 at $L_{\mathrm{x}}=1.9 \times 10^{35} \mathrm{erg} \mathrm{s}^{-1}$ (Yokogawa et al. 2003).

Survey Results: Although coverage of this source has been very complete, it has only been detected in outburst 3 times (circa MJD 53545, 53700 and 53780). Lomb-Scargle analysis of data prior to this date shows power at a period of $77.4 \mathrm{~d}$; when analysing the whole data set, the most significant peak is at $77.2 \mathrm{~d}$ (Fig. 10(b)). In both cases the ephemerides agree with the 3 outbursts (Fig. 10(a)), which strongly suggests this could be the orbital period of the system. However, it should be noted that this value is different from the reported optical periods. The ephemeris we derive is MJD $52380.5 \pm 2.3+\mathrm{n} \times 77.2 \pm 0.3 \mathrm{~d}$. The above reported $A S C A$ detections did not find the system in outburst and cannot be used to ratify this ephemeris.

\subsection{SXP15.3}

RX J0052.1-7319

RA 005214 , dec -731919

History: Lamb et al. (1999) found 15.3 s pulsations in ROSAT and BATSE data from 1996. They estimate the luminosity to be $\sim 10^{37} \mathrm{erg} \mathrm{s}^{-1}$ with a pulse fraction of $\sim 27 \%$. Edge (2005d) finds an ephemeris of MJD $50376.1+\mathrm{n} \times 75.1 \pm 0.5 \mathrm{~d}$ describes the modulation in the MACHO and OGLE light curves. It should be noted that this ephemeris is likely driven by the large 1996 X-ray outburst which is clearly visible in the OGLE data; this Type II outburst peaked around MJD 50375.

Survey Results: There was a very minor detection of SXP15.3 in February 2000 (MJD

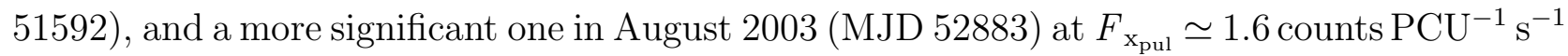
(see Fig.11). Two more bright detections separated by 13 days occurred in March 2005 (circa MJD 53445); then, on July $12^{\text {th }}$ (MJD 53564) a very bright outburst began, lasting until October $17^{\text {th }}$ (MJD 53661, 100 days long). During this time the pulsed flux oscillated between $\sim 2.0$ and $\sim 5.6$ counts $\mathrm{PCU}^{-1} \mathrm{~s}^{-1}$. A clear spin up is detected up until September $15^{\text {th }}$, when the period started increasing. The maximum period change was $\Delta P=3.3 \times 10^{-2}$ 

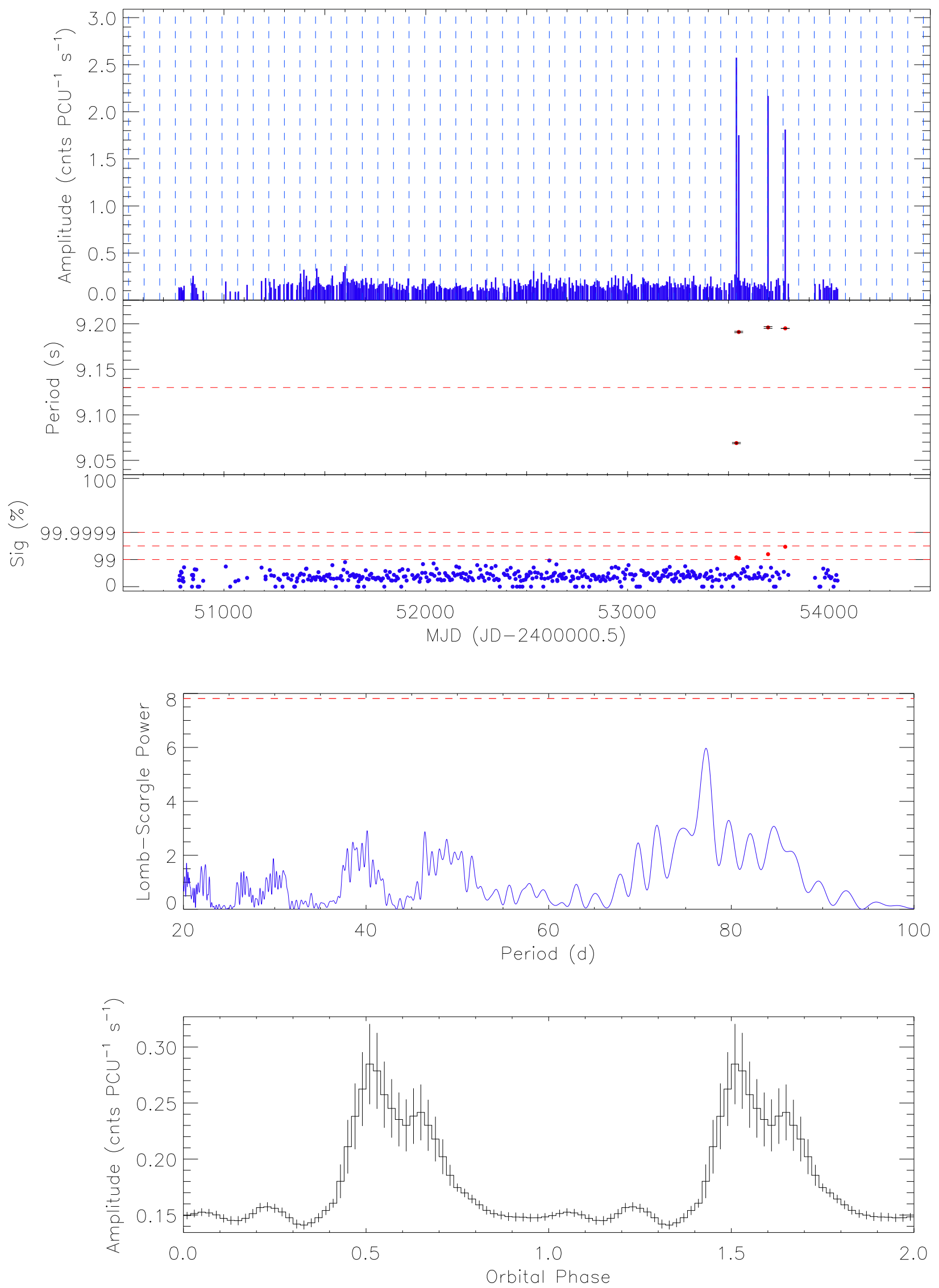

Fig. 10.- SXP9.13. a) Top: X-ray amplitude light curve. b) Middle: Lomb-Scargle power 
over the course of $56.68 \mathrm{~d}$, giving a $\dot{P}=6.74 \times 10^{-9} \mathrm{~s} \mathrm{~s}^{-1}$. We derive the expected luminosity (see Appendix B for method) from such a level of spin up (if it were all intrinsic with no orbital contribution) to be $L_{\mathrm{x}} \geq 8.6 \times 10^{37} \mathrm{erg} \mathrm{s}^{-1}\left(B \leq 1.5 \times 10^{13} \mathrm{G}\right)$.

It is likely this outburst lasted for more than one orbital cycle (expected to be $\sim 30-$ $50 \mathrm{~d}$ from the Corbet diagram) so some orbital modulation might be visible in the period data. An attempt was made to fit these data to an orbital model with constant global spin up, and also with a piece-wise approach where the spin up varies throughout the outburst (following the method outlined in Wilson et al. (2003)). Although no definite value was found, variations in the period curve suggest a period of $\sim 28 \mathrm{~d}$.

Timing analysis of the data outside the long outburst revealed no periodicities. Furthermore, the optical ephemeris proposed by Edge (2005d) does not agree with any of the detections for this source.

\subsection{SXP16.6}

\section{XTE J0050-731}

\section{No position available}

History: Discovered with a deep $121 \mathrm{ks}$ observation taken for this survey in September 2000. It was initially misidentified as RX J0051.8-7310 but later disproved Yokogawa et al. (2002), and so SXP16.6 remains unassociated with any known source, although it is often still mistakenly referred to as RX J0051.8-7310.

Survey Results: There have been a large number of detections of SXP16.6, and LombScargle analysis finds a strong modulation at 33.72d (see Fig. 12(b)), which we propose as the orbital period of the system. The ephemeris is MJD $52373.5 \pm 1.0+\mathrm{n} \times 33.72 \pm 0.05 \mathrm{~d}$.

\subsection{SXP18.3}

XTE J0055-727

\section{RA 0050 , dec -7242}

History: Reported by Corbet et al. (2003e) from observations in November/December 2003. An approximate position was established from scans with RXTE's PCA (Corbet et al. 2003e). No optical counterpart has yet been identified. A number of further outbursts between May-October 2004 provide an ephemeris of MJD $53145.7 \pm 1.3+\mathrm{n} \times 34.6 \pm 0.4 \mathrm{~d}$ from O-C (observed vs. calculated) orbital calculations (Corbet et al. 2004d). 


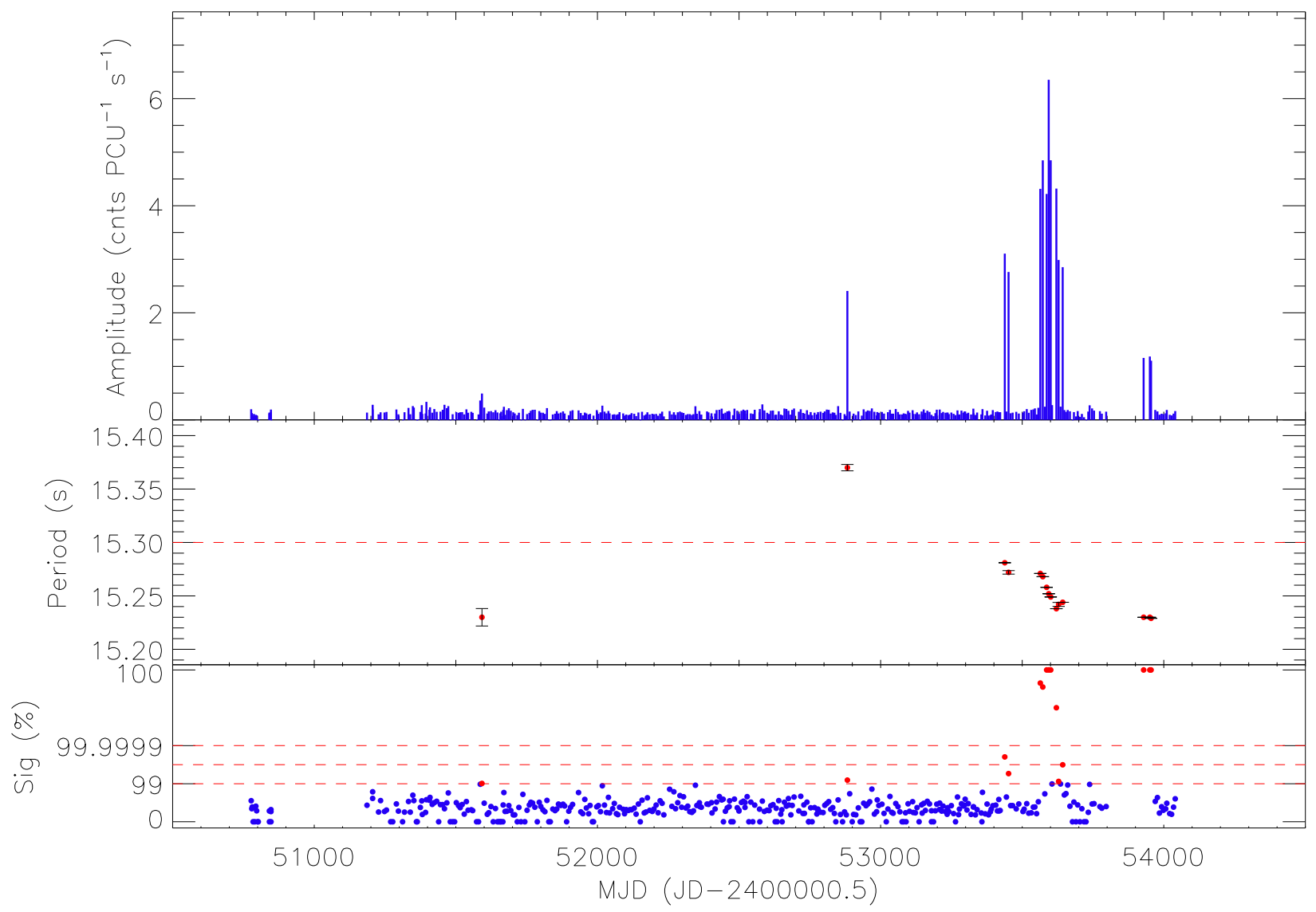

Fig. 11.- SXP15.3, X-ray amplitude light curve. 

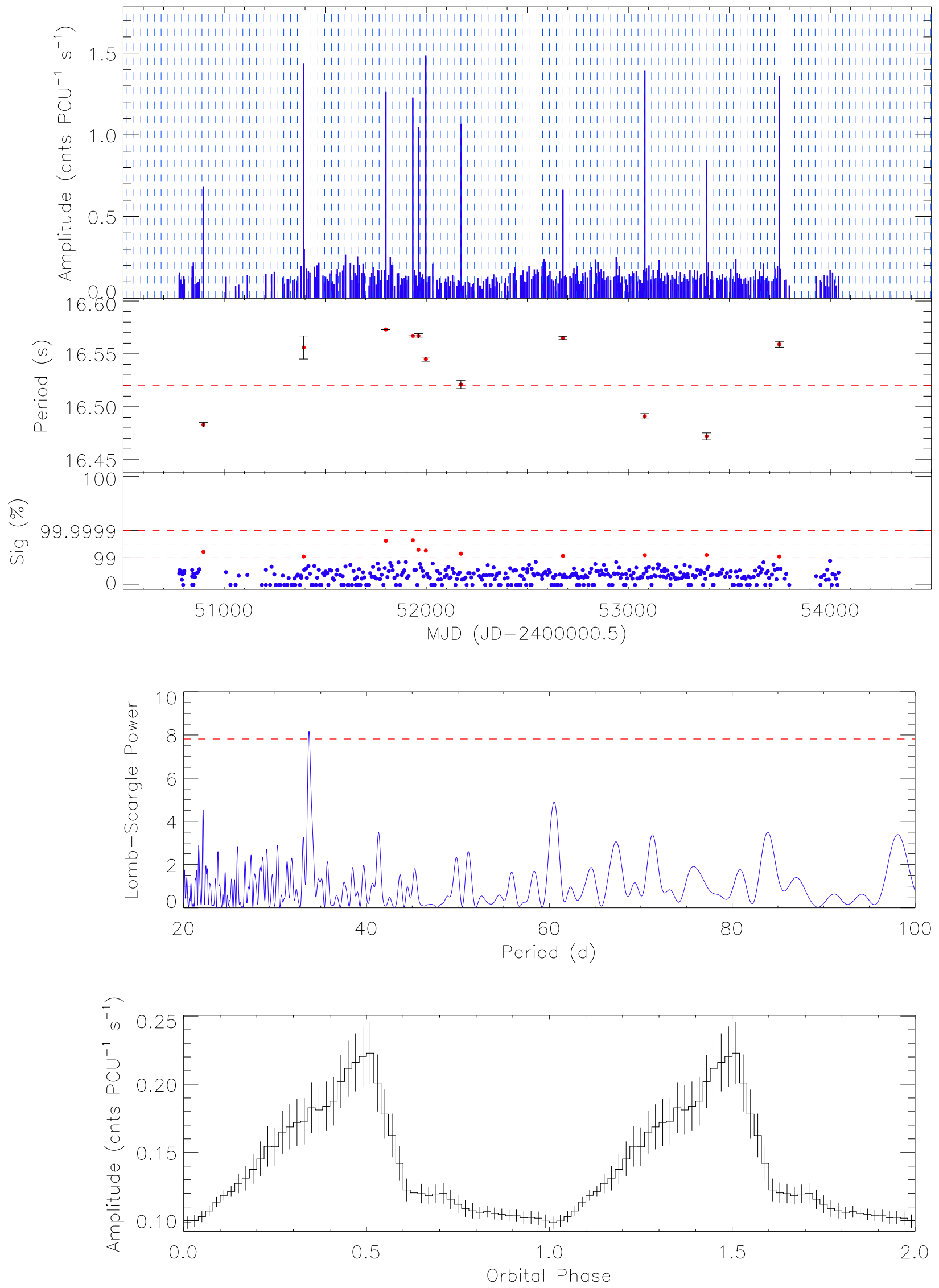

Fig. 12.- SXP16.6. a) Top: X-ray amplitude light curve. b) Middle: Lomb-Scargle power 
Survey Results: A long, bright outburst began around MJD 53925 (July 2006), which was ongoing as of January 2007 (see Fig. 13(a)). Lomb-Scargle analysis of data prior to this outburst finds a peak at $17.73 \mathrm{~d}$, which is $\sim \frac{1}{2}$ the period proposed by Corbet et al. (2004d). We find no significant power at this period and note that some of the significant detections do not agree with it. Thus, the new ephemeris for this system is MJD 52275.6 $\pm 0.9+$ $\mathrm{n} \times 17.73 \pm 0.01 \mathrm{~d}$.

\subsection{SXP22.1}

\section{RX J0117.6-7330}

\section{RA 011740.5 , dec -733052.0}

History: Discovered with ROSAT as an X-ray transient in September 1992 (Clark et al. 1996), its companion was identified as a 14.2 magnitude OB star by Charles et al. (1996). It was observed with ROSAT again in October, but no pulsations were detected (Clark et al. 1997) The companion's classification as a Be (B1-2) star was confirmed by Coe et al. (1998). $22 \mathrm{~s}$ pulsations were finally detected in ROSAT and BATSE data by Macomb et al. (1999).

Survey Results: There are only 3 observations of this pulsar's region and it was not significantly detected in any of them.

\subsection{SXP31.0}

\section{XTE J0111.2-7317, AX J0111.1-7316}

\section{RA 011109.0 , dec -731646.0}

History: Was simultaneously discovered by RXTE and BATSE during a giant outburst that began late October 1998 (Chakrabarty et al. 1998; Wilson \& Finger 1998). Schmidtke et al. (2006) report a $90.4 \mathrm{~d}$ periodicity in OGLE III data.

Survey Results: Being in the same field as SMC X-1, it was only observed 3 times, the first during the end of the aforementioned giant outburst. The two detections are $\sim 43$ days apart and a spin up of $0.4 \mathrm{~s}$ is measured, which implies a luminosity of $L_{\mathrm{x}} \geq 2.7 \times 10^{38} \mathrm{erg} \mathrm{s}^{-1}$. As Laycock et al. (2005), in an in depth spectral analysis of these observations, derive a value of $L_{\mathrm{x}} \geq 4.6 \times 10^{37} \mathrm{erg} \mathrm{s}^{-1}$, we can assume we are seeing Doppler shifted periods due to orbital motion. No further information can be derived from these observations. 

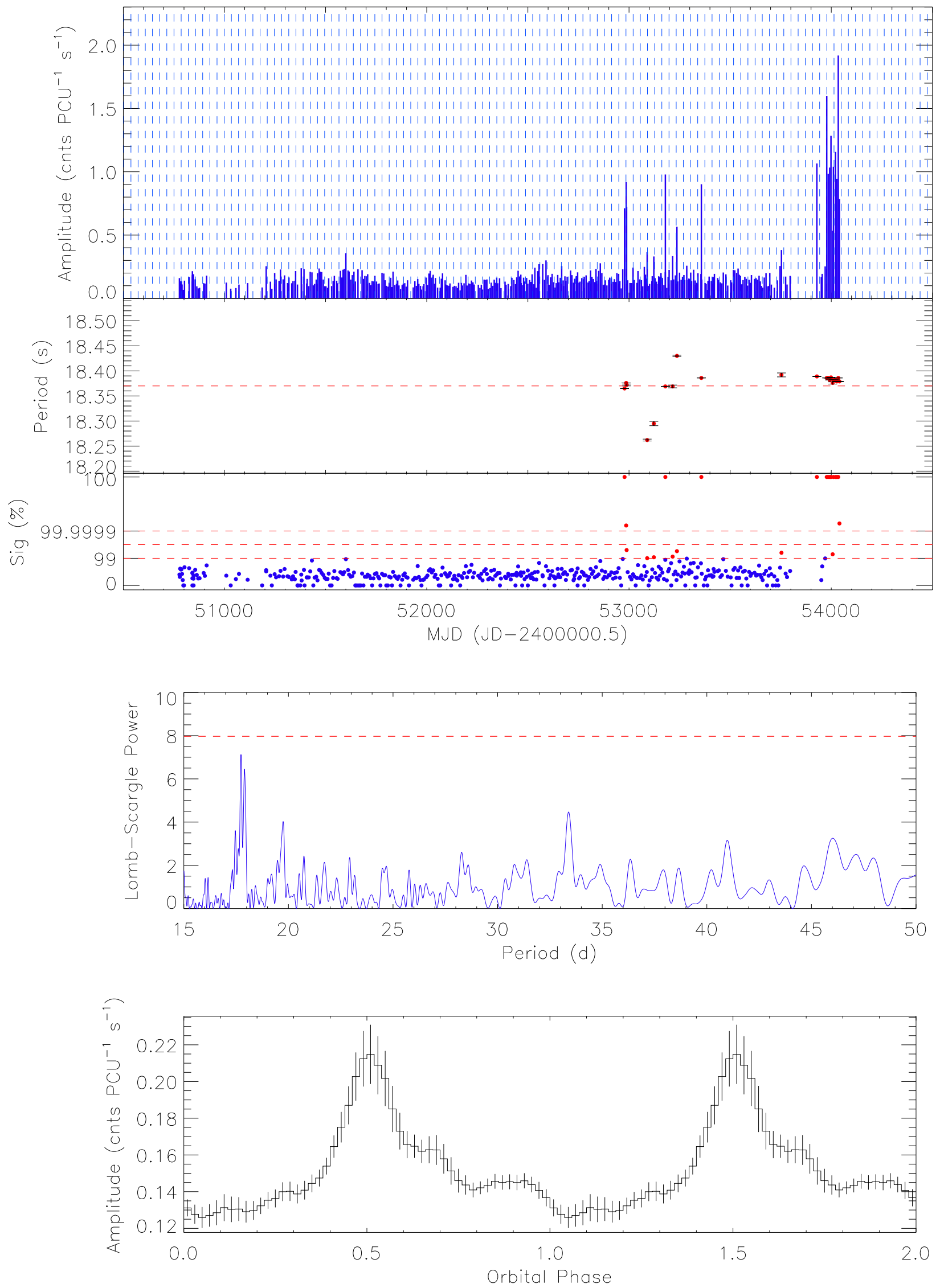

Fig. 13. - SXP18.3. a) Top: X-ray amplitude light curve (for clarity, only every other 


\subsection{SXP34.1}

RX J0055.4-7210

RA 005526.9 , dec -721059.9

History: Discovered in archival Chandra data at $34.08 \pm 0.03 \mathrm{~s}$ and lying 0.6 arcsec from a known ROSAT source (Edge et al. 2004d, c).

Survey Results: Only two significant detections are seen (MJD 50777 and 53690) and no clear periods can be found with timing analysis (see Fig. 14).

\subsection{SXP46.6}

\section{XTE J0053-724, 1WGA 0053.8-7226}

\section{RA 005358.5 , dec -722635}

History: Discovered in the first observation of this survey (November 25 $5^{\text {th }} 1998$ ) with a period of $46.63 \pm 0.04 \mathrm{~s}$ while it was undergoing a long outburst (Corbet et al. 1998). Laycock et al. (2005) derive a period of $139 \pm 6 \mathrm{~d}$ from 6 X-ray outbursts in the earlier part of this survey. Two candidates for the optical counterpart were proposed by Buckley et al. (2001). Schmidtke et al. (2007) confirm it is star B and find two periods in OGLE data: $69.2 \pm 0.3 \mathrm{~d}$ and $138.4 \pm 0.9 \mathrm{~d}$; they suggest the possibility that the orbital period is the shorter or the two values.

Survey Results: The source was thought to be inactive after January 2002. In the meantime a new SMC pulsar with a $46.4 \mathrm{~s}$ period was announced (Corbet et al. 2002). LombScargle analysis of both pulsars revealed the same orbital periods and very similar ephemeris, suggesting they were the same pulsar which has been slowly spinning up (Galache et al. 2005). The estimated luminosity required for a spin up of $\dot{P}=1.05 \times 10^{-9} \mathrm{~s} \mathrm{~s}^{-1}$ during MJD $50800-51900$ is $L_{\mathrm{x}} \geq 9.9 \times 10^{35} \mathrm{erg} \mathrm{s}^{-1}\left(B \leq 6.0 \times 10^{12} \mathrm{G}\right)$, and for a $\dot{P}=4.68 \times 10^{-10} \mathrm{~s} \mathrm{~s}^{-1}$ during MJD, $L_{\mathrm{x}} \geq 4.4 \times 10^{35} \mathrm{erg} \mathrm{s}^{-1}\left(B \leq 4.0 \times 10^{12} \mathrm{G}\right)$. The ephemeris of the outbursts is now best described by MJD $52293.9 \pm 1.4+\mathrm{n} \times 137.36 \pm 0.35 \mathrm{~d}$ (see Fig. 15)(a)). Although the folded light curve shows a small increase in flux half a phase away from maximum, we do not believe this is evidence that the orbital period is half the calculated value. Given the clear, regular outbursts experienced by this system throughout the survey we believe the true orbital period is $\sim 137 \mathrm{~d}$. 


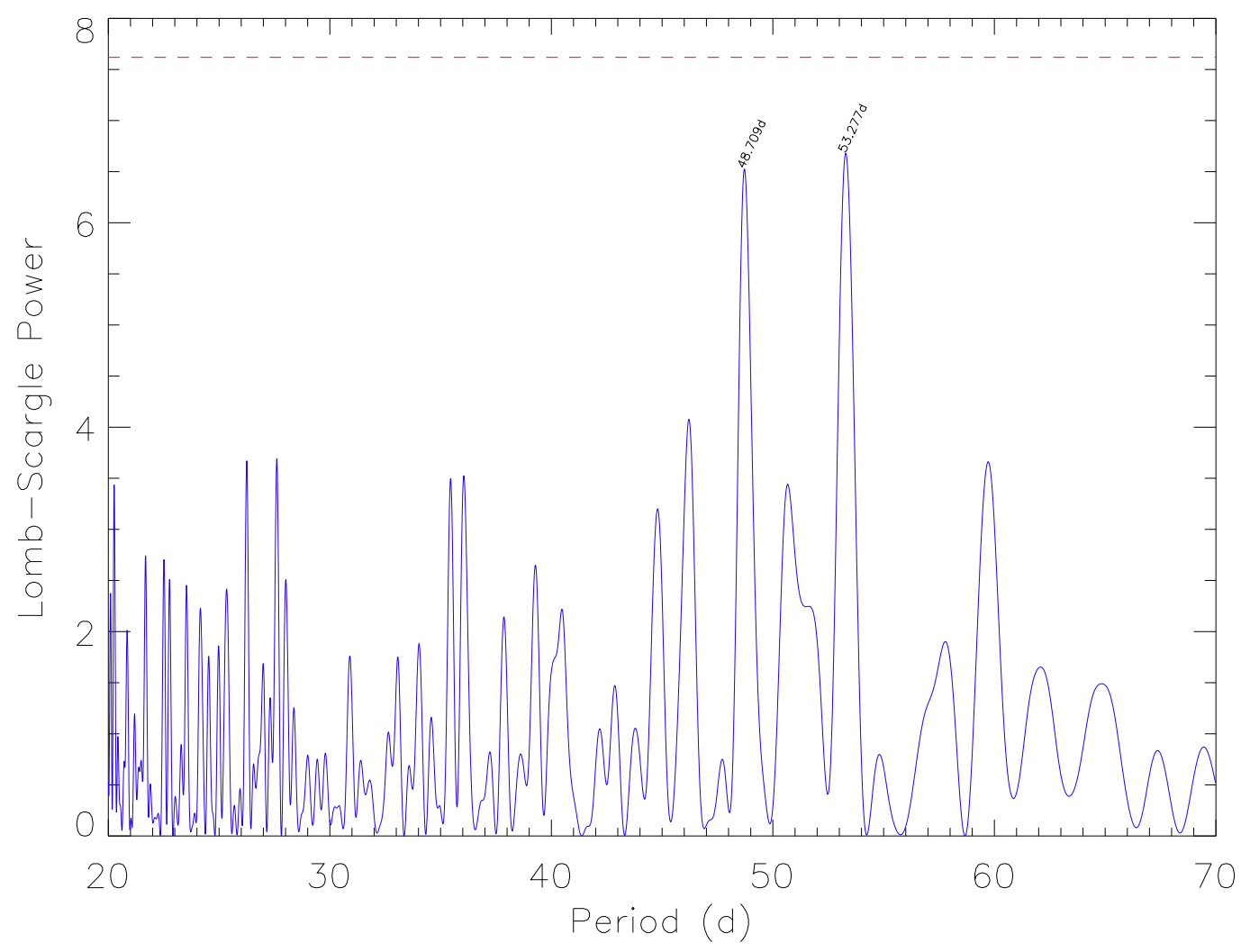

Fig. 14.- SXP34.1, X-ray amplitude light curve. 

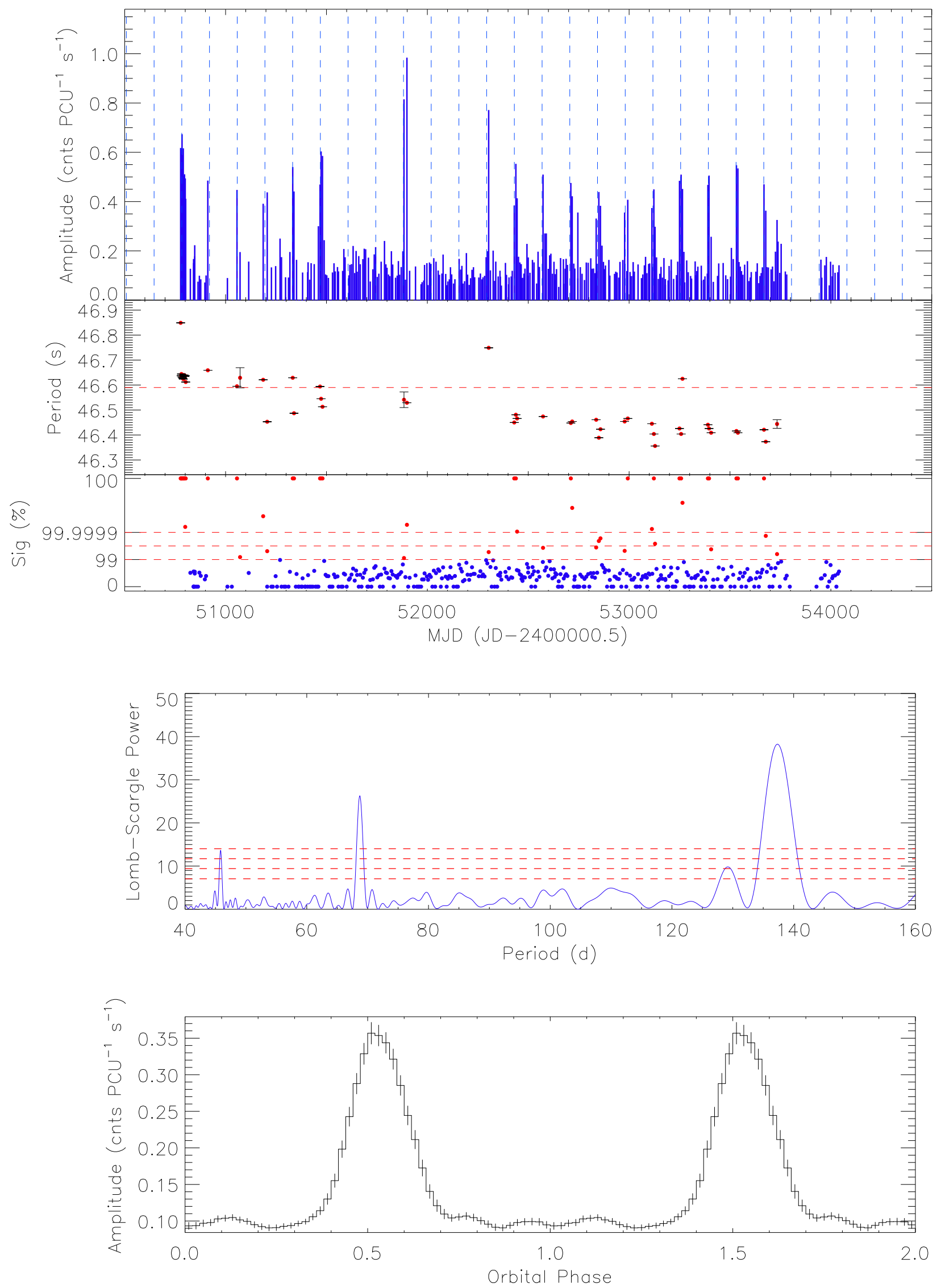

Fig. 15.- SXP46.6. a) Top: X-ray amplitude light curve. b) Middle: Lomb-Scargle power 


\subsection{SXP51.0}

\section{SMC 51}

\section{No position available}

History: Was erroneously proposed as a new 25.5 s pulsar in Lamb et al. (2002) from a deep $121 \mathrm{ks}$ observation. Laycock (2002) identifies the $25.5 \mathrm{~s}$ peaks in the power spectrum as harmonics of SXP51.0's true pulse period. No position is available; it lies within Position 4, and most likely in the overlap between Positions $1 / \mathrm{A}$ and 4.

Survey Results: Despite numerous significant detections (see Fig. 16), timing analysis finds no strong periodicities in the light curve.

\subsection{SXP59.0}

\section{XTE J0055-724, RX J0054.9-7226, 1WGA J0054.9-7226}

\section{RA 005456.6 , dec -722650}

History: Discovered in RXTE observations of the vicinity of SMC X-3 (Marshall et al. 1998), it showed 4 bright, very similar, outbursts during January 1998-September 1999 from which Laycock et al. (2005) derived an orbital period of $123 \pm 1 \mathrm{~d}$. The optical counterpart was established by Stevens et al. (1999); Schmidtke \& Cowley (2005b) found a $60.2 \pm 0.8 \mathrm{~d}$ period from timing analysis of OGLE and MACHO data which they propose as the orbital period.

Survey Results: SXP59.0 remained undetected from September 1999 until a bright outburst in mid 2002 (circa MJD 52520) kicked off a series of 5 outbursts (see Fig. 17(a)). From the whole data range we extract an ephemeris of MJD $52306.1 \pm 3.7+\mathrm{n} \times 122.10 \pm 0.38 \mathrm{~d}$. We note that this period is twice the optical period but we detect no significant flux half a phase from maximum that would suggest the orbital period is not 122 days.

During the 1998-99 outbursts a spin up was detected of $\dot{P}=4.7 \times 10^{-9} \mathrm{ss}^{-1}$ and again throughout the 5 outbursts of $2002-2003$, with a value of $\dot{P}=5.9 \times 10^{-9} \mathrm{~s} \mathrm{~s}^{-1}$. The luminosities associated with these spin ups are, respectively, $L_{\mathrm{x}} \geq 2.6 \times 10^{36} \mathrm{erg} \mathrm{s}^{-1}(B \leq$ $\left.1.3 \times 10^{13} \mathrm{G}\right)$ and $L_{\mathrm{x}} \geq 3.3 \times 10^{36} \mathrm{erg} \mathrm{s}^{-1}\left(B \leq 1.4 \times 10^{13} \mathrm{G}\right)$. In between both groups of outbursts SXP59.0 was observed to have spun down during the $\sim 1100$ days it was undetected. The average spin down was $\dot{P}=-4.2 \times 10^{-9} \mathrm{~s} \mathrm{~s}^{-1}$, which would be associated with an estimated $L_{\mathrm{x}} \geq 2.9 \times 10^{36} \mathrm{erg} \mathrm{s}^{-1}$. Although SXP59.0 was further away from the center of the field of view during AO5-AO6 (MJD 51600-52300), it should have been picked up as a number of the detections during the second outburst season were made when $R X T E$ was 


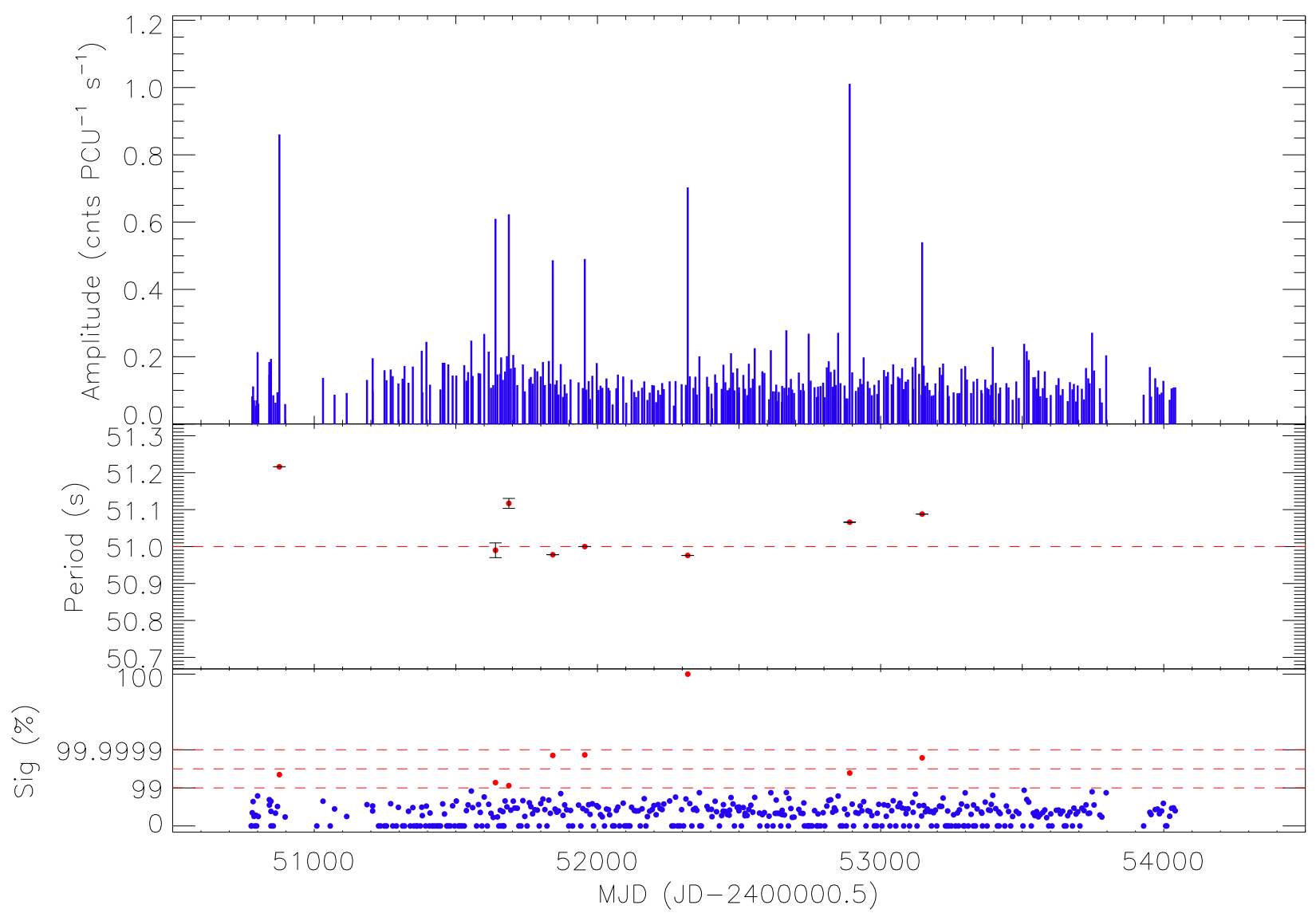

Fig. 16. - SXP51.0, X-ray amplitude light curve. 
pointing at Position D (essentially the same coordinates as Position 5). In view of this, the spin down mechanism for SXP59.0 must be something other than reverse accretion torque and is likely due to the propeller effect.

\subsection{SXP65.8}

\section{CXOU J010712.6-723533}

\section{RA 0107 12.63, dec -723533.8}

History: Discovered as part of a Chandra survey of the SMC wing that is reported in McGowan et al. (2007). They detected a source at $L_{\mathrm{x}}=3 \times 10^{36} \mathrm{erg} \mathrm{s}^{-1}(37 \pm 5 \%$ pulse fraction) with pulsations at $65.78 \pm 0.13 \mathrm{~s}$. Its position was found to coincide with the emission line star [MA93] 1619, a $V=16.6$ Be star. A $110.6 \mathrm{~d}$ period has been observed in the MACHO data for this object (Schmidtke \& Cowley 2007b).

Survey Results: Due to its location outside the heavily observed positions, this pulsar has very little data. We find no significant detections and no periodic modulation is apparent in the X-ray light curve.

\subsection{SXP74.7}

RX J0049.1-7250, AX J0049-729

\section{RA 004904 , dec -725054}

History: Discovered in the first observation of this survey with a period of $74.8 \pm 0.4 \mathrm{~s}$ (Corbet et al. 1998). Kahabka \& Pietsch (1998) identified it with the ROSAT source RX J0049.1-7250 and Stevens et al. (1999) found a single Be star within the ROSAT error radius which they proposed as the optical counterpart. Only 3 X-ray outbursts where observed in the early stages of this survey (before MJD 52300), from which Laycock et al. (2005) derived a possible orbital period of $642 \pm 59 \mathrm{~d}$ based on the separation between the outbursts. Schmidtke \& Cowley (2005b) and Edge (2005d) find a 33.4 d periodicity in OGLE data.

Survey Results: Lomb-Scargle analysis of the data finds a period at $\sim 62 \mathrm{~d}$, which although not strong, shows a convincing profile (see Fig. 18(b)); its ephemeris is MJD 52319.0 $\pm 3.1+$

$\mathrm{n} \times 61.6 \pm 0.2 \mathrm{~d}$. There is a weak peak in the power spectrum at $33.2 \mathrm{~d}$, close to the reported optical period, but folding the light curve at this period does not produce a clear modulation. 

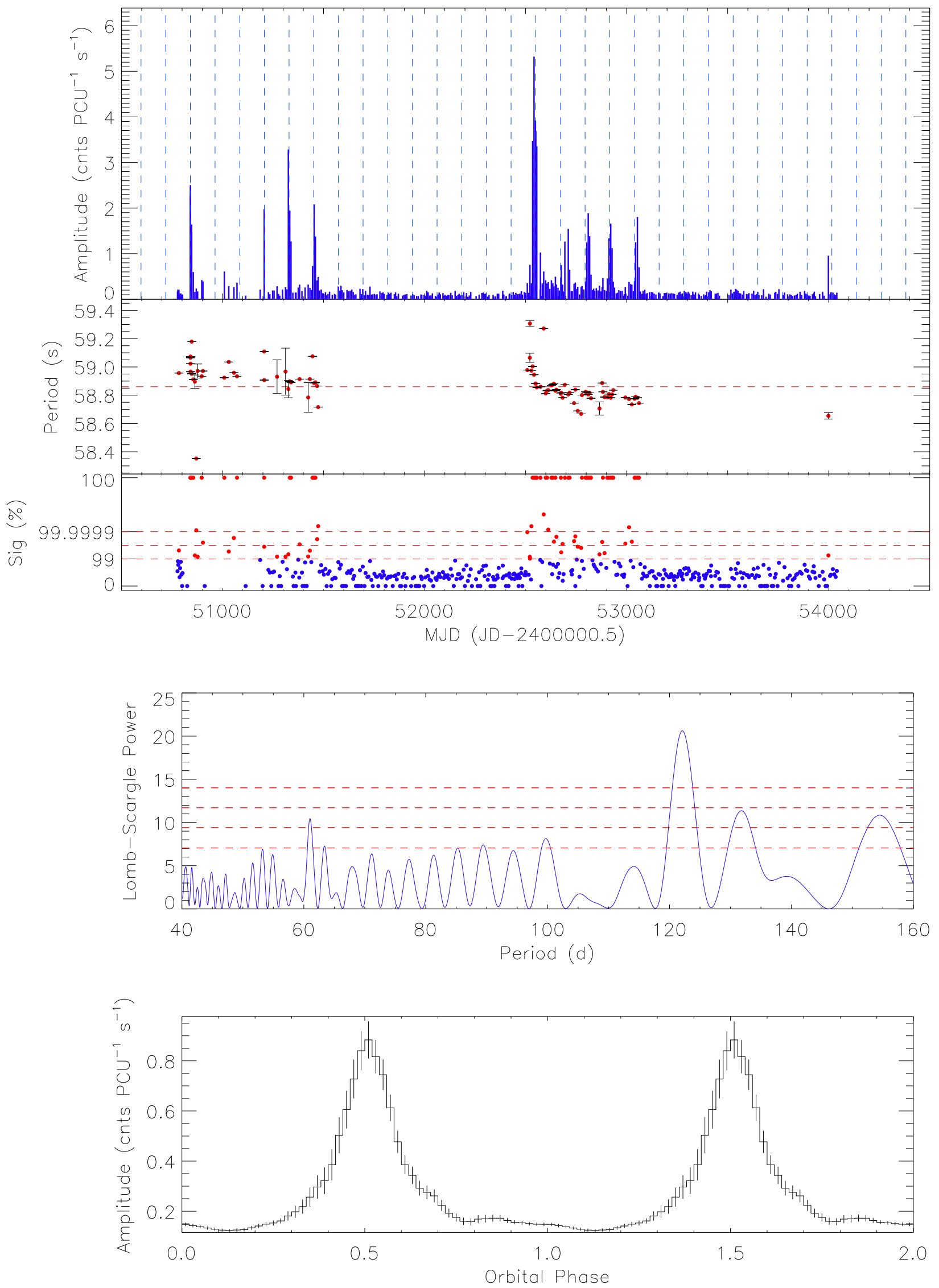

Fig. 17.- SXP59.0. a) Top: X-ray amplitude light curve. b) Middle: Lomb-Scargle power 

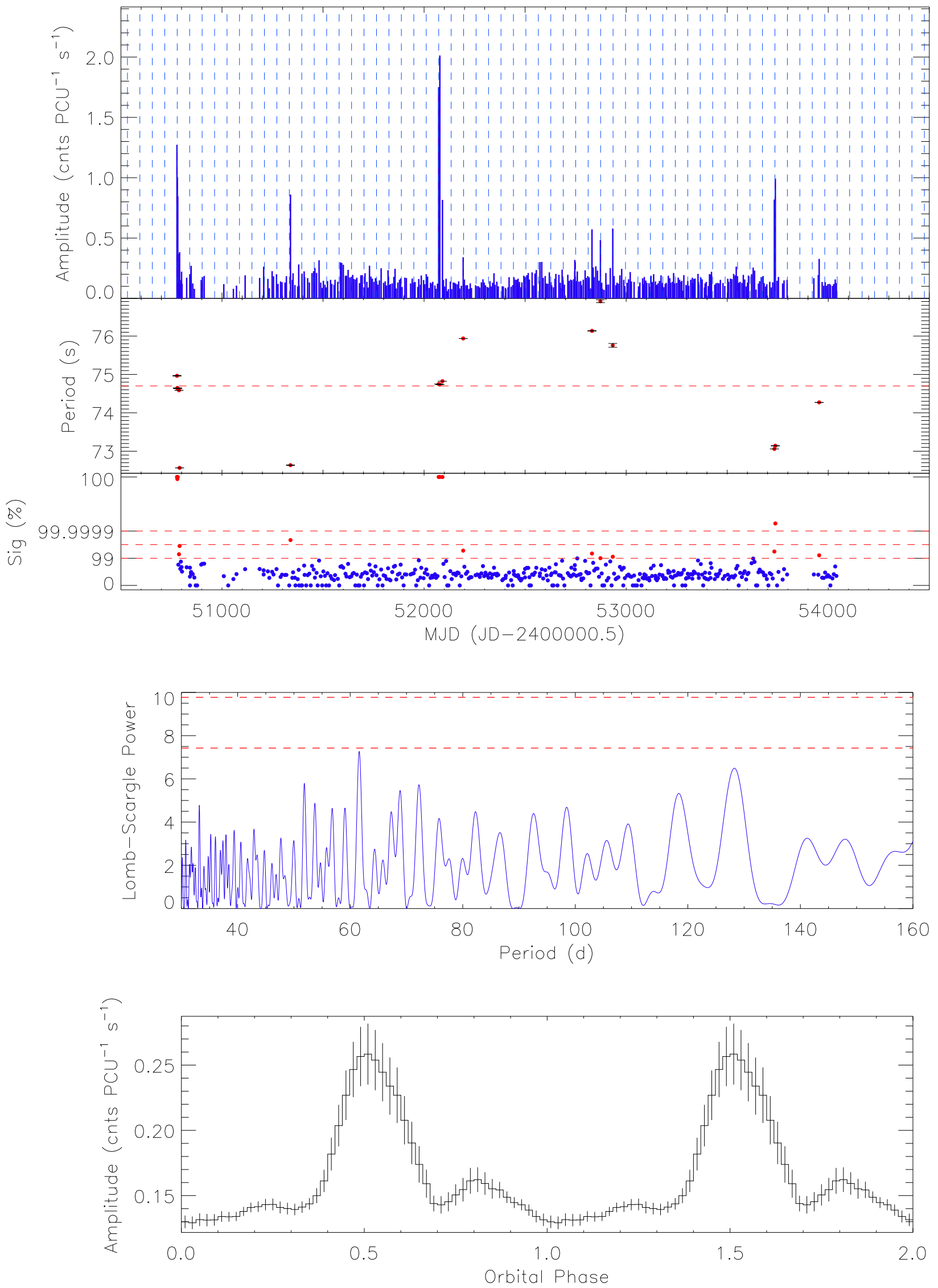

Fig. 18. - SXP74.7. a) Top: X-ray amplitude light curve. b) Middle: Lomb-Scargle power 


\subsection{SXP82.4}

XTE J0052-725

RA 005209 , dec -723803

History: First observed by RXTE in this survey (Corbet et al. 2002), its position was determined from archival Chandra observations (Edge et al. 2004b). OGLE data show a strong modulation at $\sim 380 \mathrm{~d}$ (Edge 2006, private communication).

Survey Results: The Lomb-Scargle periodogram for the light curve shows a very significant peak at $\sim 362 \mathrm{~d}$ with a number of harmonics (see Fig. 19(b)); the ephemeris derived is MJD $52089.0 \pm 3.6+\mathrm{n} \times 362.3 \pm 4.1 \mathrm{~d}$. Although this period is longer than would be expected given its $P_{\mathrm{s}}$ position in the Corbet diagram, its similarity to the optical period would seem to confirm it is the actual orbital period of the system. We note that in the aforementioned Chandra observation of this puslar on MJD 52459, it was detected at $L_{\mathrm{x}}=3.4 \times 10^{36} \mathrm{erg} \mathrm{s}^{-1}$ (Edge et al. 2004c); this date is $\sim 8$ days after our predicted periastron passage and the luminosity exhibited is consistent with a Type I outburst (which was also detected with RXTE see Fig. 19(a)).

\subsection{SXP89.0}

\section{XTE SMC pulsar}

\section{No position available}

History: Reported by Corbet et al. (2004b) from observations in March 2002, it is located within the field of view of Position 1/A.

Survey Results: The first outburst is a single detection in February 2000 (MJD 51592), 2 years before the official discovery; 4 others occurred 2 years later in a short space of timewithin $\sim 260$ days (see Fig. 20(a)). They follow a high-low-high-low brightness pattern, with very similar high/low countrates. The separation between them is $\sim 88 \mathrm{~d}$ which could be expected to be the orbital period. In fact, timing analysis finds a strong period at $87.6 \mathrm{~d}$, with an ephemeris of MJD $52337.5 \pm 6.1+\mathrm{n} \times 87.6 \pm 0.3 \mathrm{~d}$.

\subsection{SXP91.1}

AX J0051-722, RX J0051.3-7216

RA 005055 , dec -721338 

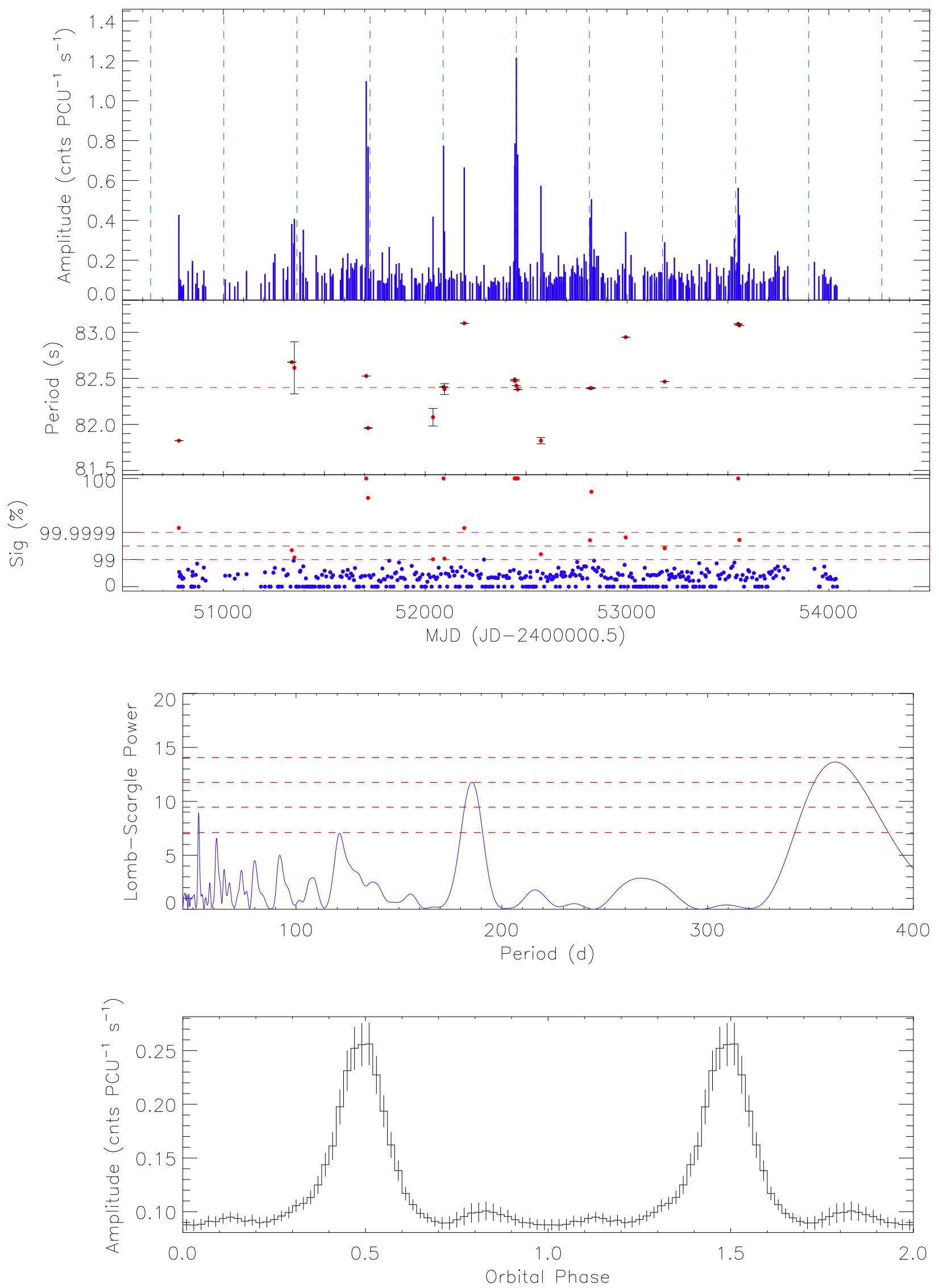

Fig. 19.- SXP82.4. a) Top: X-ray amplitude light curve. b) Middle: Lomb-Scargle power 

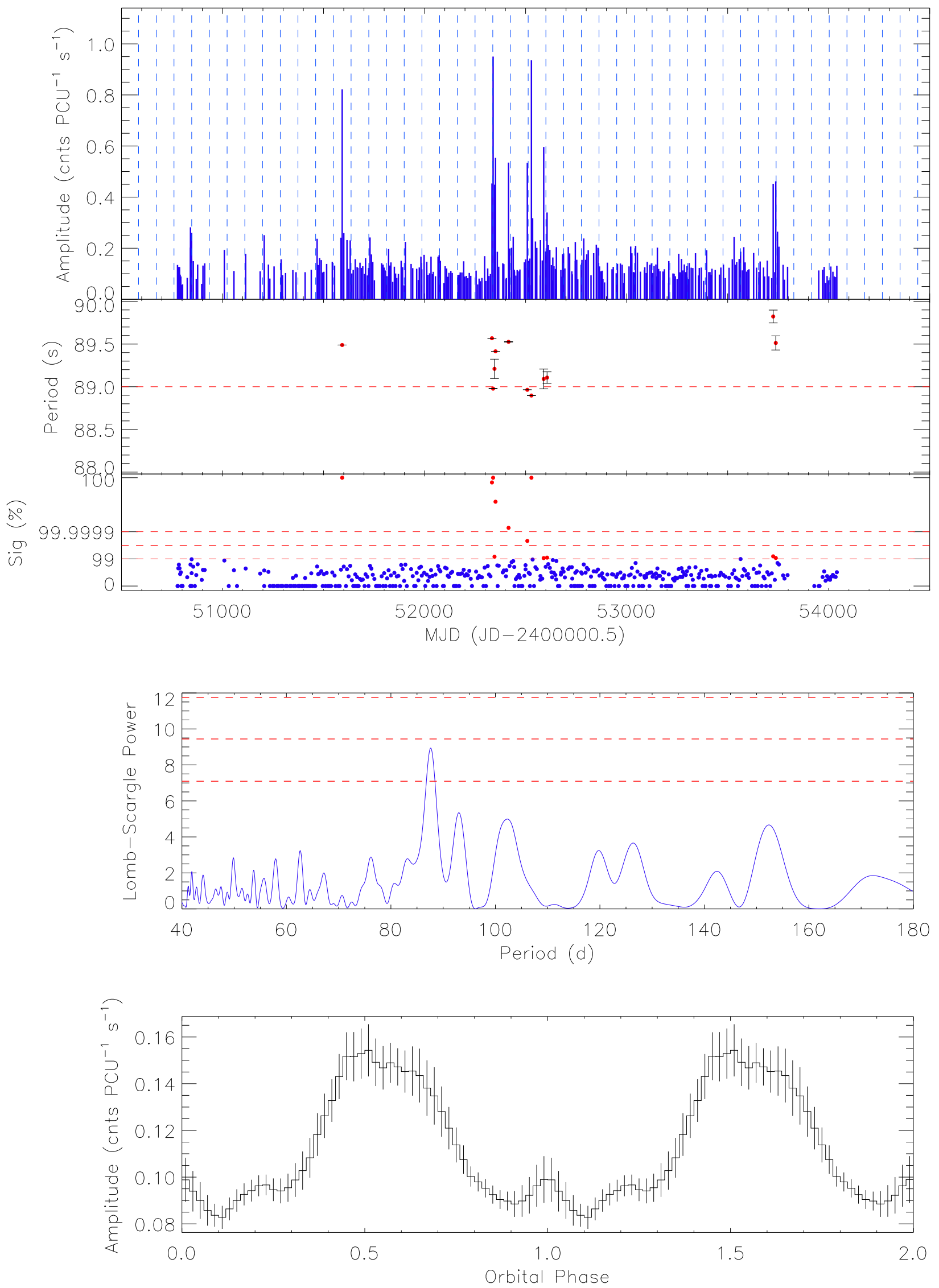

Fig. 20.- SXP89.0. a) Top: X-ray amplitude light curve. b) Middle: Lomb-Scargle power 
History: Discovered in the first observation in this survey with a period of $92 \pm 1.5 \mathrm{~s}$ (Marshall et al. 1997), further analysis improved this measurement to $91.12 \pm 0.05 \mathrm{~s}$ (Corbet et al. 1998). An orbital period of $115 \pm 5 \mathrm{~d}$ was derived by Laycock et al. (2005) from early survey data (before MJD 52200). Stevens et al. (1999) identified the optical counterpart; Schmidtke et al. (2004) find an 88.25 d period in their analysis of MACHO data for this star.

Survey Results: Corbet et al. (2004b) reported the discovery of a new $89 \mathrm{~s}$ pulsar from $X T E$ observations in March 2002, it was located within the field of view of Position 1/A. After studying the long-term lightcurves of these two pulsars, we believe SXP89.0 is actually SXP91.1 after having spun up (see Fig. 21(a)). The Lomb-Scargle periodogram of the whole light curve returns a period of $\sim 101 \mathrm{~d}$, which is slightly shorter than the period found by Laycock et al. (2005). Timing analysis of the light curve post MJD 52300 shows no clear periods. The ephemeris we derive is MJD $52197.9 \pm 8.2+\mathrm{n} \times 117.8 \pm 0.5 \mathrm{~d}$. The average spin up during MJD $50750-51550$ is calculated to be $\dot{P}=1.8 \times 10^{-8} \mathrm{~s} \mathrm{~s}^{-1}$, with a luminosity of $L_{\mathrm{x}} \geq 3.6 \times 10^{36} \mathrm{erg} \mathrm{s}^{-1}\left(B \leq 2.5 \times 10^{13} \mathrm{G}\right)$.

Given the proximity in spin period between SXP91.1 and SXP89.0, and the fact that the optical orbital period of SXP91.1 is so similar to the X-ray period of SXP89.0, it is possible that they may be one and the same pulsar. In Fig. 22(a) we show the consolidated light curve for both pulsars showing the possibility that SXP91.1 spun up sufficiently to be later detected as a separate pulsar. We are hesitant to claim they are one single system because the orbital ephemeris of this consolidated data set does not coincide with either system's ephemeris: MJD 52301.2 $\pm 3.0+\mathrm{n} \times 101.3 \pm 0.4 \mathrm{~d}$. However, the orbital profile (see Fig. 22(b)) is convincing and appears similar to that of SXP8.88 (Fig. 9(b)) or SXP18.3 (Fig. 13(b)).

\subsection{SXP95.2}

\section{SMC 95}

\section{RA 0052 , dec -7245}

History: Was discovered in March 1999 in data from this survey (Laycock et al. 2002); an approximate position was obtained with PCA scans over the source and has a large uncertainty. The X-ray orbital period suggested from the two bright outbursts before MJD 52300 is $283 \pm 8 \mathrm{~d}$ (Laycock et al. 2005).

Survey Results: Only 3 other marginal detections are available in the data (see Fig. 23). Lomb-Scargle analysis does not return any clear period, either including or excluding the two bright outbursts, although the former has its highest power at $71.3 \mathrm{~d}$, an ephemeris that 

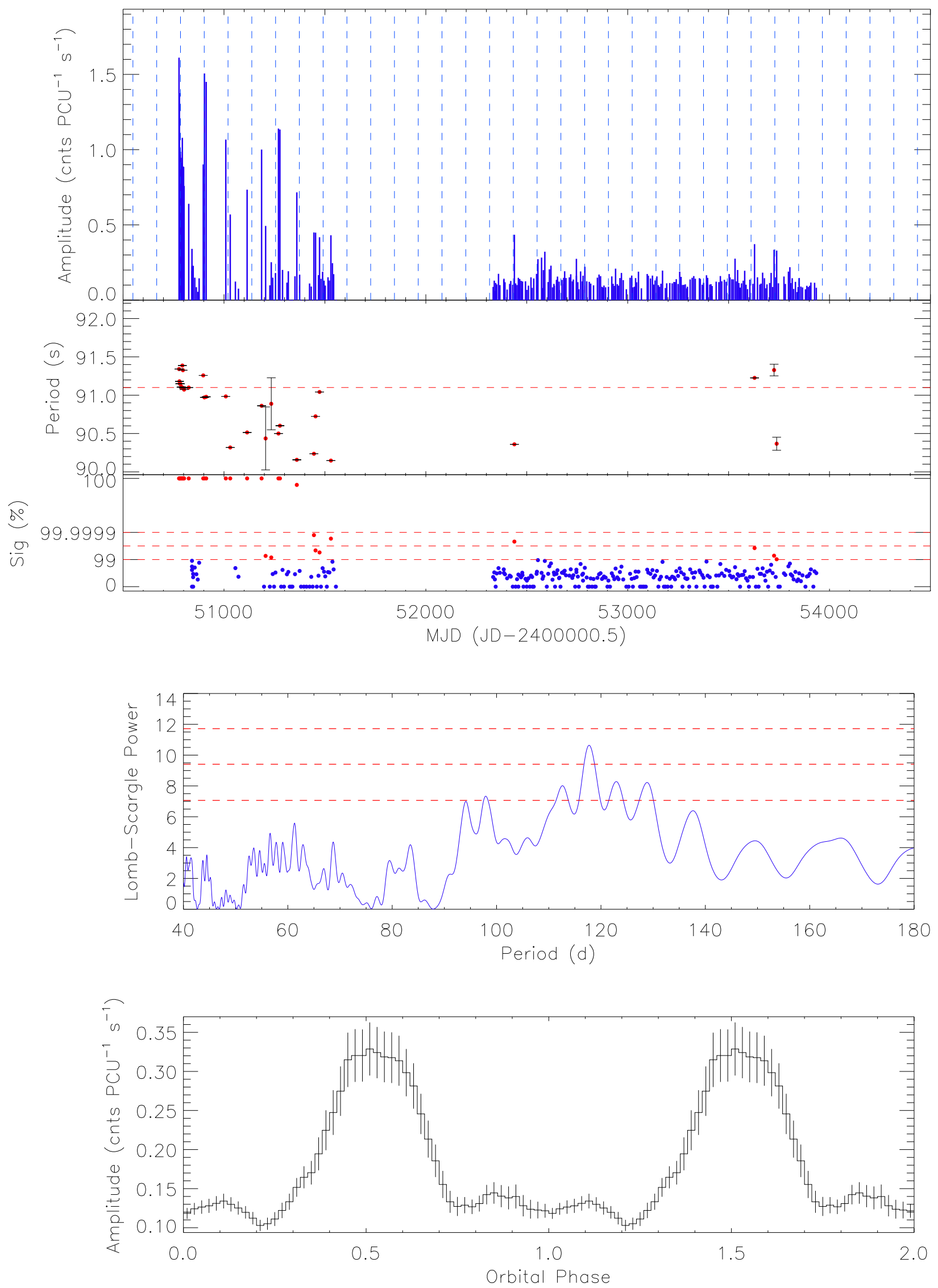

Fig. 21.- SXP91.1. a) Top: X-ray amplitude light curve. b) Middle: Lomb-Scargle power 

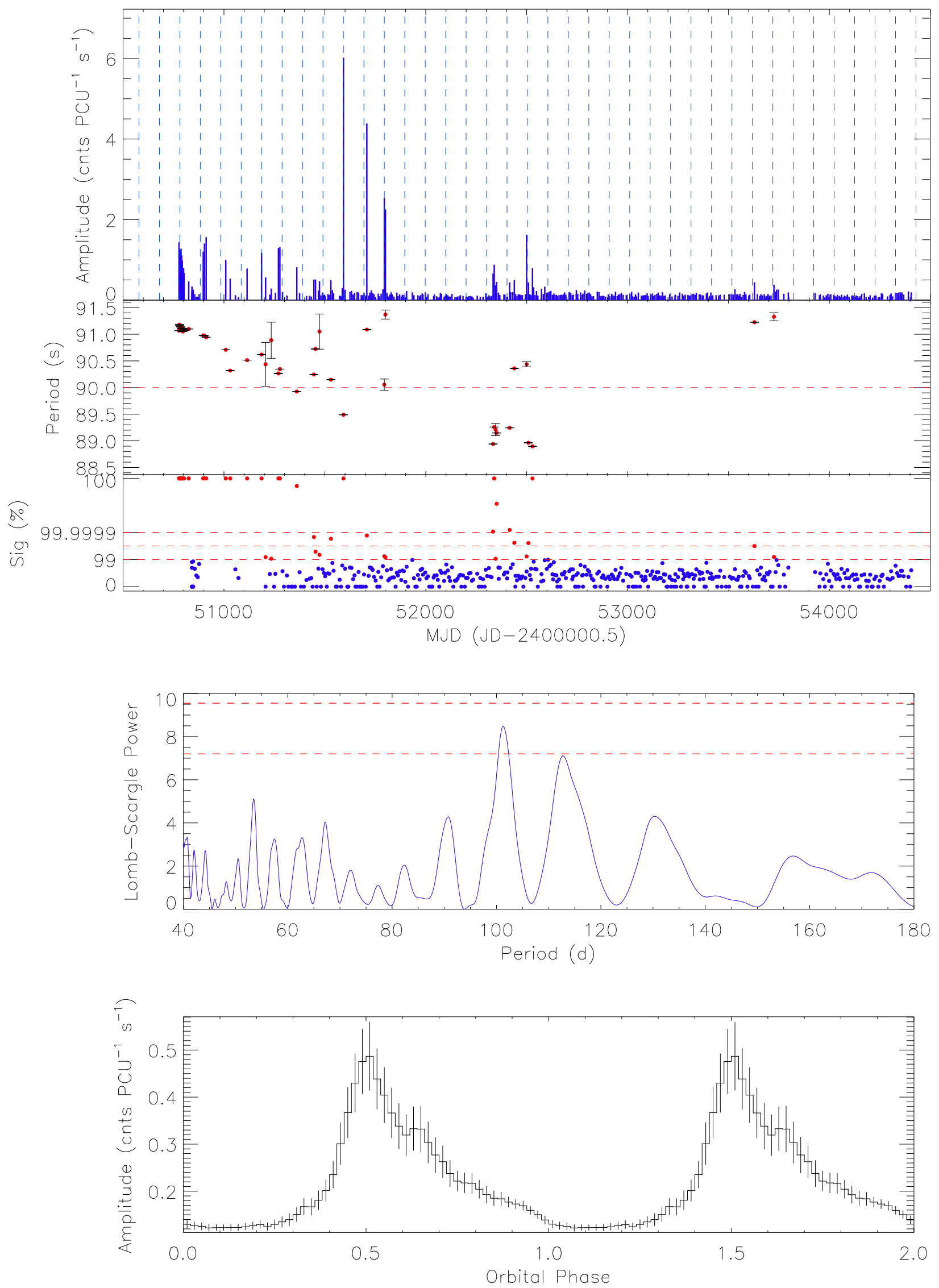

Fig. 22.- Consolidated light curve of SXP89.0 and SXP91.1. a) Top: X-ray amplitude light 
is consistent with the source's significant detections and may reflect its orbital period.

\subsection{SXP101}

RX J0057.3-7325, AX J0057.4-7325

RA 005726.8 , dec -732502

History: Discovered in an $A S C A$ observation at a period of $101.45 \pm 0.07 \mathrm{~s}$ (Yokogawa et al. 2000a), and identified also as a ROSAT source. Two optical counterparts were suggested by Edge \& Coe (2003); from a Chandra observation McGowan et al. (2007) pinpoint the counterpart as a $V=14.9$ star that exhibits a $21.9 \mathrm{~d}$ periodicity in both OGLE III and MACHO data. Schmidtke \& Cowley (2007b) find the same period.

Survey Results: This pulsar lies in the SE edge of the wing and was in the field of view of Positions 4 and 5, so coverage is only continuous throughout AO5 and AO6 (see Fig. 24); during this time only 3 outbursts of low brightness were observed (MJD 51814, 51863 and 52137). Timing analysis returns no significant periods, although the highest peak in the periodogram is at $25.2 \mathrm{~d}$, similar to the optical period.

\subsection{SXP138}

CXOU J005323.8-722715

\section{RA 005323.8 , dec -722715.0}

History: Discovered in archival Chandra data (Edge et al. 2004b), the optical counterpart is [MA93] 667 (Edge 2005d). The MACHO light curves for the companion star reveal peaks at $\sim 125.1 \mathrm{~d}$ intervals (stronger in the red band). Schmidtke \& Cowley (2006) report finding a weak periodicity in the $122-123$ s region in $\mathrm{MACHO}$ data.

Survey Results: X-ray data from this survey show two brighter detections $\sim 112 \mathrm{~d}$ apart (see Fig. 2.28(a)). Lomb-Scargle analysis finds no significant periods; however, if these two detections are removed from the data, a period of $103.6 \mathrm{~d}$ appears in the power spectrum. Its ephemerides (shown by the vertical, dashed lines in Fig. 2.28(a)) do not correspond with the two detections, and the profile (see bottom panel of Fig. 2.28(b)) looks almost sinusoidal, unlike the profiles exhibited by other systems in the SMC. The maxima for this period occur at MJD $52400.2 \pm 5.2+\mathrm{n} \times 103.6 \pm 0.5 \mathrm{~d}$. 


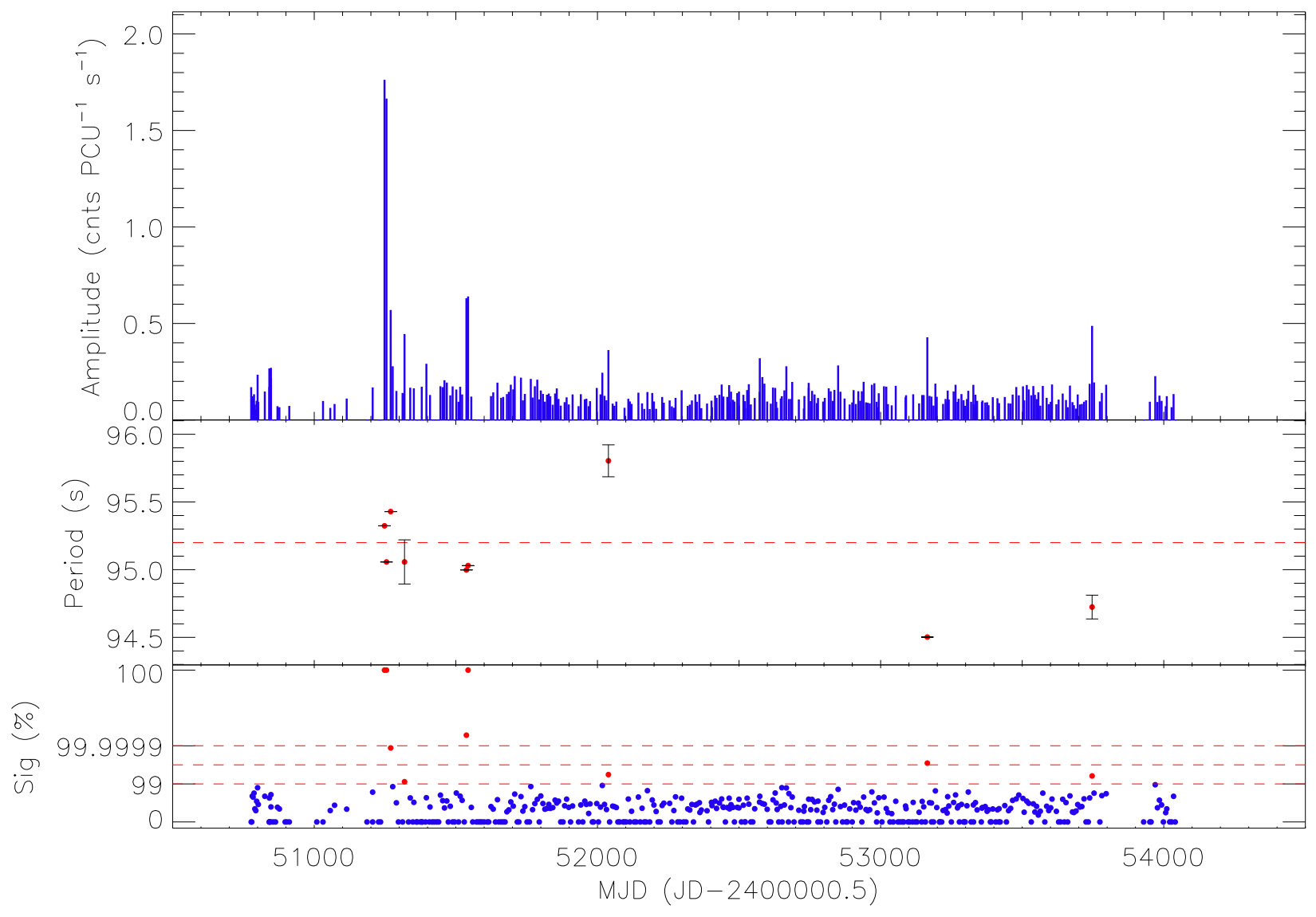

Fig. 23.- SXP95.2, X-ray amplitude light curve. 


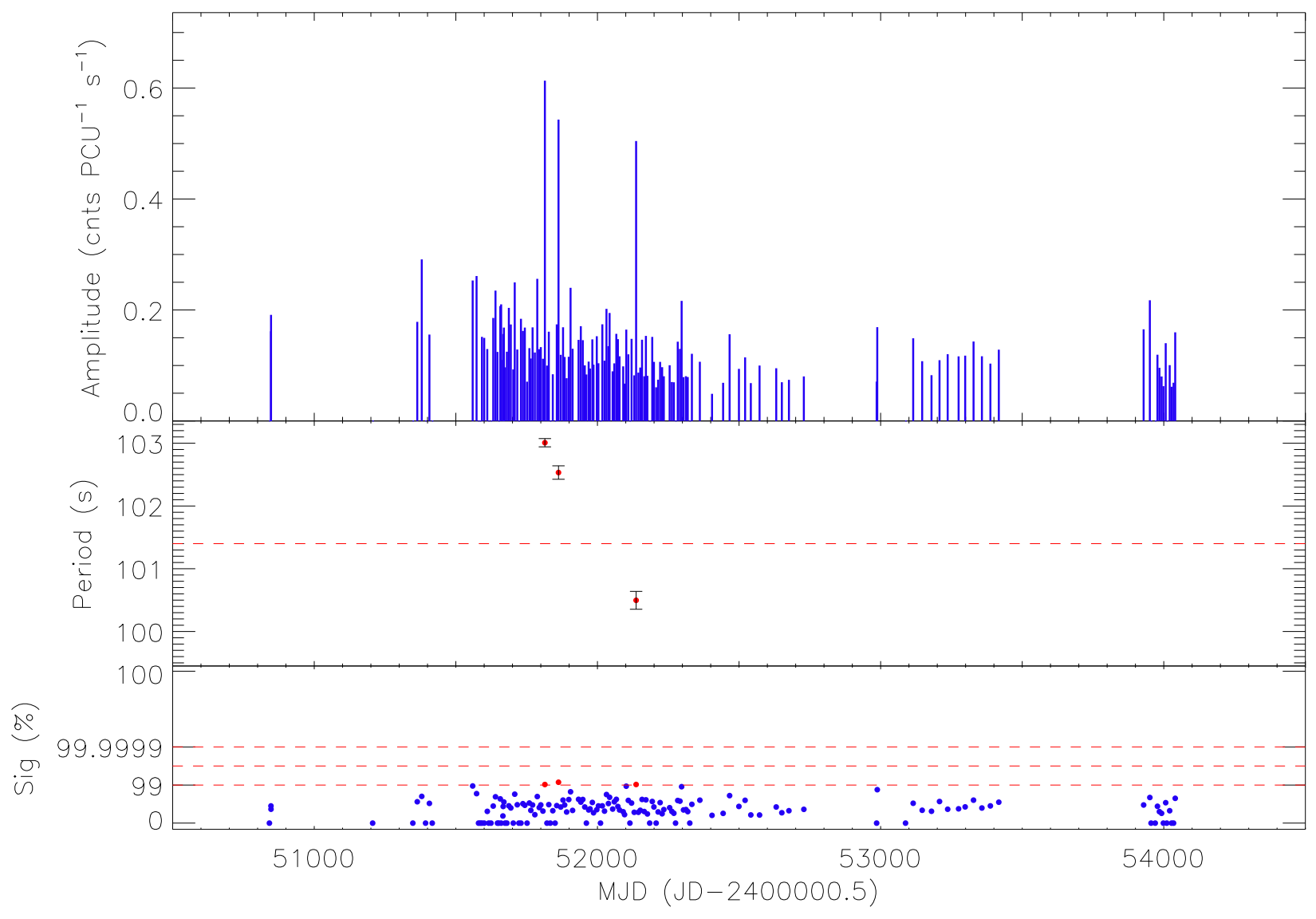

Fig. 24.- SXP101, X-ray amplitude light curve. 

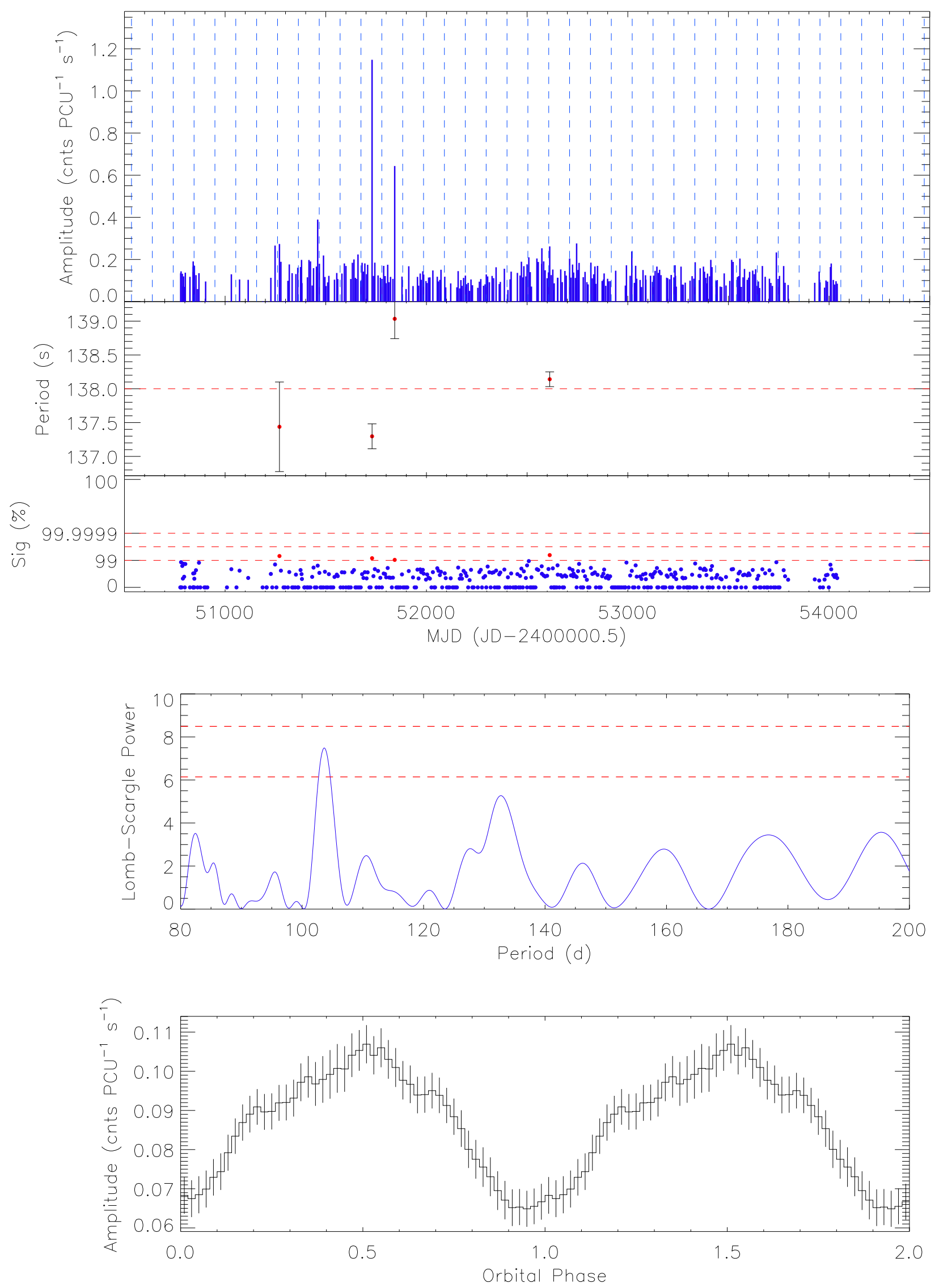

Fig. 25.- SXP138. a) Top: X-ray amplitude light curve. b) Middle: Lomb-Scargle power 


\subsection{SXP140}

XMMU J005605.2-722200, 2E0054.4-7237

\section{RA 005605.7 , dec -722200}

History: Discovered in XMM observations by Sasaki et al. (2003). The optical counterpart is believed to be [MA93] 904 (Haberl \& Pietsch 2004). Schmidtke \& Cowlev (2006) find a $197 \pm 5 \mathrm{~d}$ period in MACHO data.

Survey Results: None of the detections has been longer than 1 week, with only 2 of them showing significant brightness (see Fig. 26). Timing analysis returns no clear periods, although the periodogram of the data, excluding the two bright detections, does show some power at $\sim 197 \mathrm{~d}$. As a number of different periods have similar power, this may only be coincidence. The folded light curve at $197 \mathrm{~d}$ does not show a typical orbital profile.

\subsection{SXP144}

\section{XTE SMC pulsar}

\section{No position available}

History: Detected in observations from this survey in April 2003 by Corbet et al. (2003b), who later reported an ephemeris of MJD $52779.2 \pm 2.9+\mathrm{n} \times 61.2 \pm 1.6 \mathrm{~d}$ (Corbet et al. 2003d).

Survey Results: Although there are a few minor detections before the initial discovery, April 2003 ( MJD 52750) saw the beginning of a regular pattern of outbursts which continued until February 2006 ( MJD 53800, see Fig.27(a)). The neutron star has displayed an extremely linear and constant spin down during this time, with an average $\dot{P}=1.6 \times 10^{-8} \mathrm{~s} \mathrm{~s}^{-1}$, from which we derive a $L_{\mathrm{x}} \geq 1.1 \times 10^{36} \mathrm{erg} \mathrm{s}^{-1}\left(B \leq 2.4 \times 10^{13} \mathrm{G}\right)$. The improved outburst ephemeris from Lomb-Scargle analysis is MJD $52368.9 \pm 1.8+\mathrm{n} \times 59.38 \pm 0.09 \mathrm{~d}$. This period is shorter than might have have expected from the pulse-orbit relationship, but what is most unusual about this pulsar is that it is spinning down, moving it even further away from the Be group on the Corbet diagram.

\subsection{SXP152}

CXOU J005750.3-720756

RA 005749 , dec -720759 


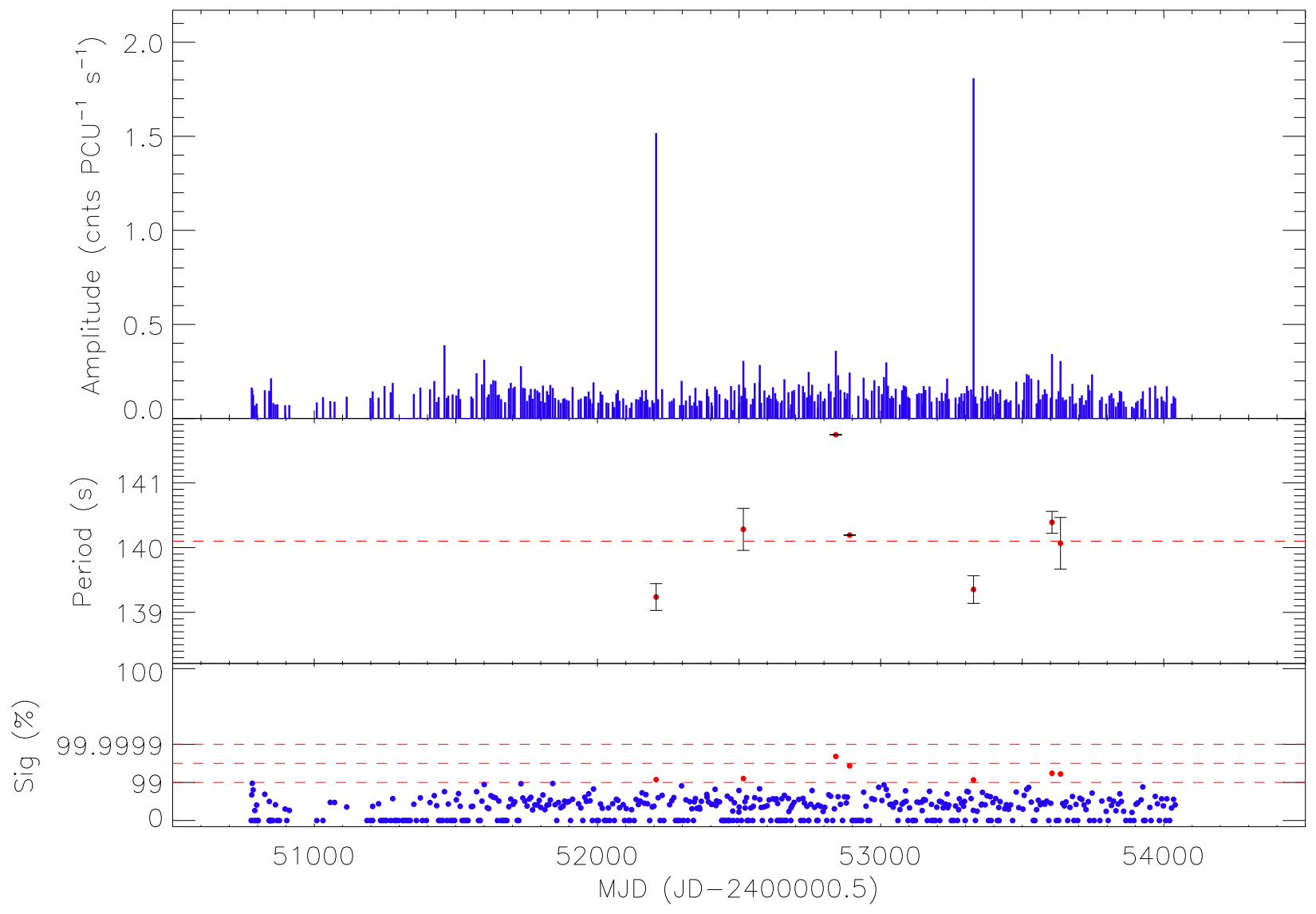

Fig. 26. - SXP140, X-ray amplitude light curve. 

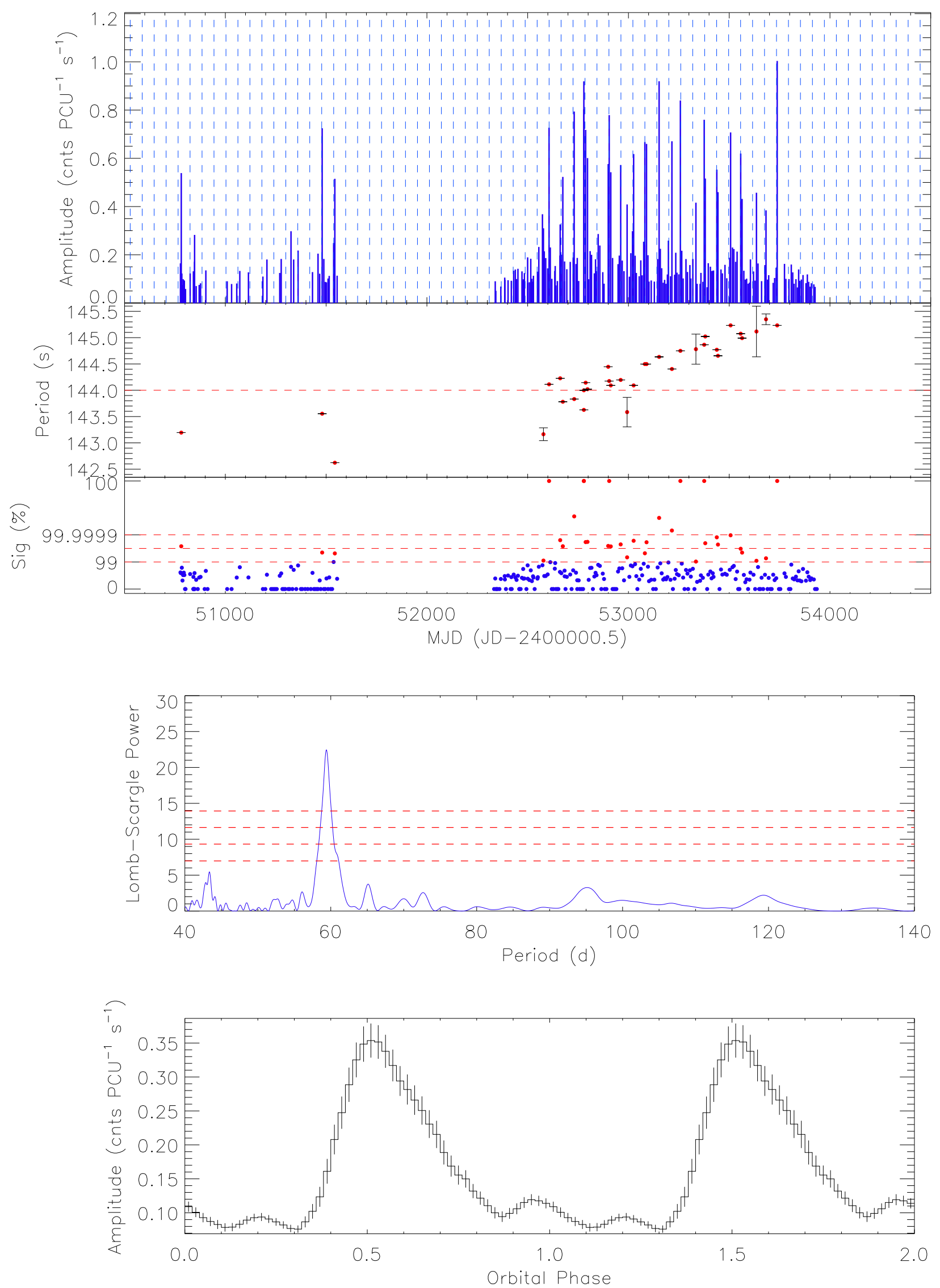

Fig. 27.- SXP144. a) Top: X-ray amplitude light curve. b) Middle: Lomb-Scargle power 
History: Haberl \& Sasaki (2000) suggested that this object is a Be binary pulsar based on the $\mathrm{H} \alpha$-emitting object [MA93] 1038, although ROSAT observations of this source had not detected any pulsations. These were found in a long Chandra observation by Macomb et al. (2003) at a period of $152.098 \pm 0.016 \mathrm{~s}$ (they report a very high pulse fraction of $64 \pm 3 \%$ and a $\left.L_{\mathrm{x}}=2.6 \times 10^{35} \mathrm{erg} \mathrm{s}^{-1}\right)$, and in an XMM observation by Sasaki et al. (2003) at $152.34 \pm 0.05 \mathrm{~s}$.

Survey Results: The observational history is shown in Fig. 28. The periodogram of the light curve shows no clear orbital period, although the highest power peak is at $\sim 107 \mathrm{~d}$, which would agree with the expected value from the Corbet diagram. The lack of periodic outbursts, despite its clear X-ray activity, may point towards a low eccentricity system. This would limit accretion onto the neutron star to times when the Be star ejecta are dense enough, and would be independent of the orbital phase. Analysis of the optical light curve of the companion Be star is needed.

\subsection{SXP169}

XTE J0054-720, AX J0052.9-7158, RX J0052.9-7158

\section{RA 005254.0 , dec -715808.0}

History: First detected by RXTE in December 1998 at a period of $169.30 \mathrm{~s}$ (Lochner et al. 1998). Laycock et al. (2005) suggested a possible orbital period of $200 \pm 40 \mathrm{~d}$. Galache et al. (2005) reported an orbital period of $68.6 \pm 0.2 \mathrm{~d}$ based on data from this survey while Schmidtke et al. (2006) found a period of $67.6 \pm 0.3 \mathrm{~d}$ in OGLE -III data.

Survey Results: Corbet et al. (2004b) announced a new SMC pulsar at $164.7 \mathrm{~s}$, with an unknown position. After comparing the long term light curves and the ephemerides from timing analysis it became apparent that SXP165 and SXP169 were the same source. A consolidated light curve is presented here (Fig. 29(a)), where the spin up of SXP169 throughout the survey is apparent. The estimated spin up during MJD $50800-51500$ is $\dot{P}=$ $2.5 \times 10^{-8} \mathrm{~s} \mathrm{~s}^{-1}$, implying $L_{\mathrm{x}} \geq 1.2 \times 10^{36} \mathrm{erg} \mathrm{s}^{-1}\left(B \leq 3.0 \times 10^{13} \mathrm{G}\right)$; for the remaining data the spin up is $\dot{P}=2.0 \times 10^{-8} \mathrm{~s} \mathrm{~s}^{-1}$, implying $L_{\mathrm{x}} \geq 9.6 \times 10^{35} \mathrm{erg} \mathrm{s}^{-1}\left(B \leq 2.6 \times 10^{13} \mathrm{G}\right)$. LombScargle analysis provides a clear period, and the outbursts are described by the ephemeris MJD $52240.1 \pm 2.1+\mathrm{n} \times 68.54 \pm 0.15 \mathrm{~d}$, in agreement with the reported optical period.

\subsection{SXP172}

AX J0051.6-7311, RX J0051.9-7311

RA 005152 , dec -731035 


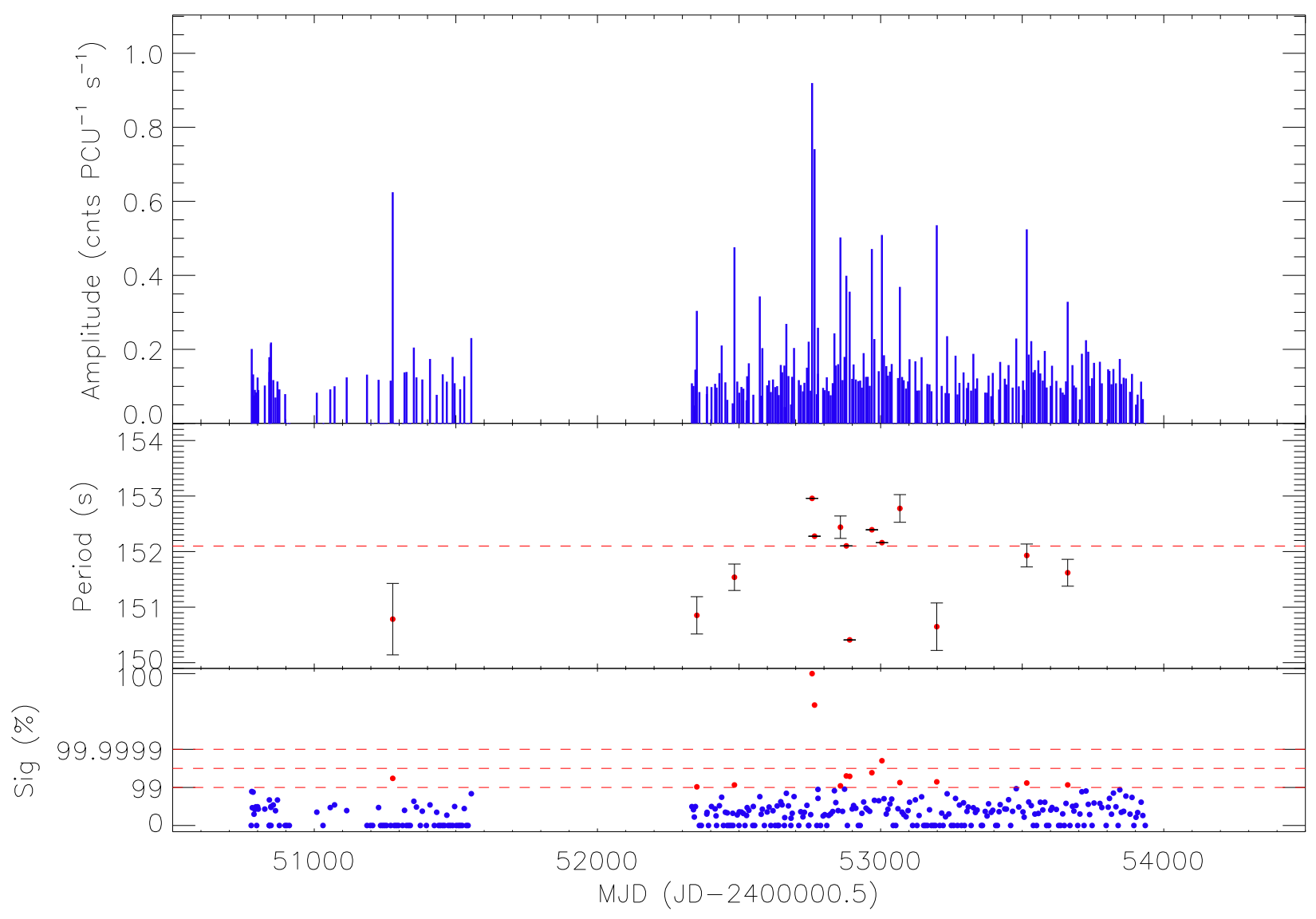

Fig. 28.- SXP152, X-ray amplitude light curve. 

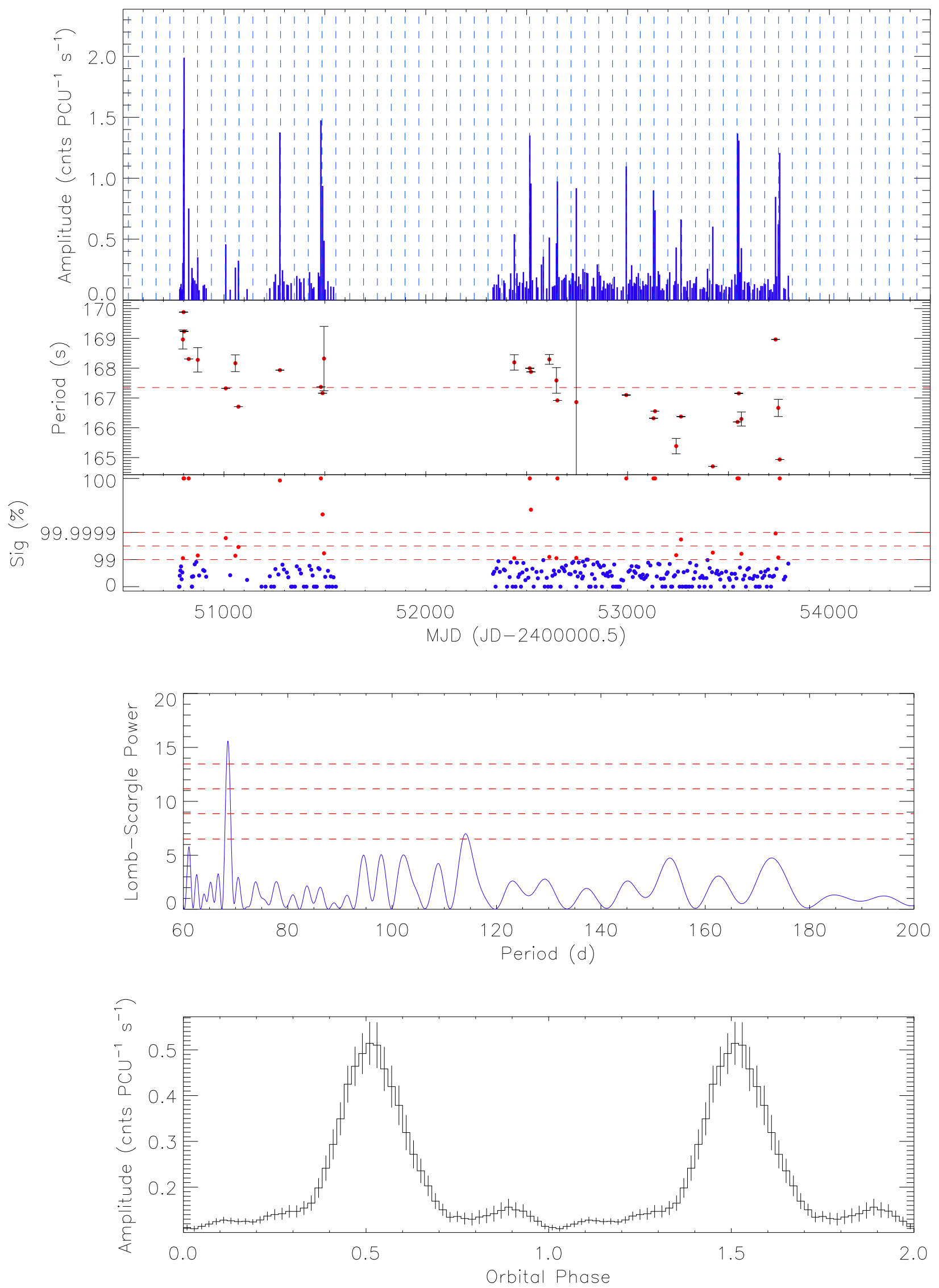

Fig. 29.- SXP169. a) Top: X-ray amplitude light curve. b) Middle: Lomb-Scargle power 
History: Found in an $A S C A$ observation (Torii et al. 2000), it was identified with the ROSAT source RX J0051.9-7311, which has a Be optical counterpart (Cowley et al. 1997). Laycock et al. (2005) suggest a possible orbital period of $\sim 67 \mathrm{~d}$ based on the X-ray activity up until MJD 52350. Schmidtke \& Cowley (2006) report an optical period of $69.9 \pm 0.6 \mathrm{~d}$ in OGLE II data. This pulsar has been detected on 17 occasions by EINSTEIN, ROSAT and $A S C A$, but never above $L_{\mathrm{x}}=7.8 \times 10^{35} \mathrm{erg} \mathrm{s}^{-1}$ (Yokogawa et al. 2000c).

Survey Results: SXP172 underwent a phase of intense, semi-regular, activity during MJD 51600-52400 (see Fig. 30). Lomb-Scargle analysis was carried using only data from this period, and also the whole data set, with no conclusive outcome. However, it is clear that the series of outbursts during the aforementioned dates are separated by $\sim 70$ days (consistent with the optical period). It is possible we are seeing contamination from another pulsar of similar pulse period, maybe located within a different pointing position. As past missions did not detect this pulsar in outburst, it is hard to confirm an X-ray ephemeris.

\subsection{SXP202}

XMMU J005920.8-722316

\section{RA 005920.8 , dec -722316}

History: Detected in a number of archival $X M M$ observations and reported in Majid et al. (2004); the authors found an early B type star at the X-ray coordinates and classified it as a HMXB.

Survey Results: This source has shown little bright activity throughout the survey except for the outburst in January 2006 (circa MJD 53740, see Fig. 31). Lomb-Scargle analysis of the data returns no clear period, but we note that a $\sim 91 \mathrm{~d}$ orbital period would agree with the 6 outburst detections since MJD 53000.

\subsection{SXP264}

\section{XMMU J004723.7-731226, RX J0047.3-7312, AX J0047.3-7312}

\section{RA 004723.7 , dec -731225}

History: It was initially reported by Yokogawa et al. (2003) from ASCA observations, although it had previously been detected (yet remained undiscovered) in earlier $X M M$ observations (Ueno et al. 2004). It had originally been proposed as a Be/X-ray binary candidate by Haberl \& Sasaki (2000) based on its X-ray variability. Edge et al. (2005a) identified the com- 


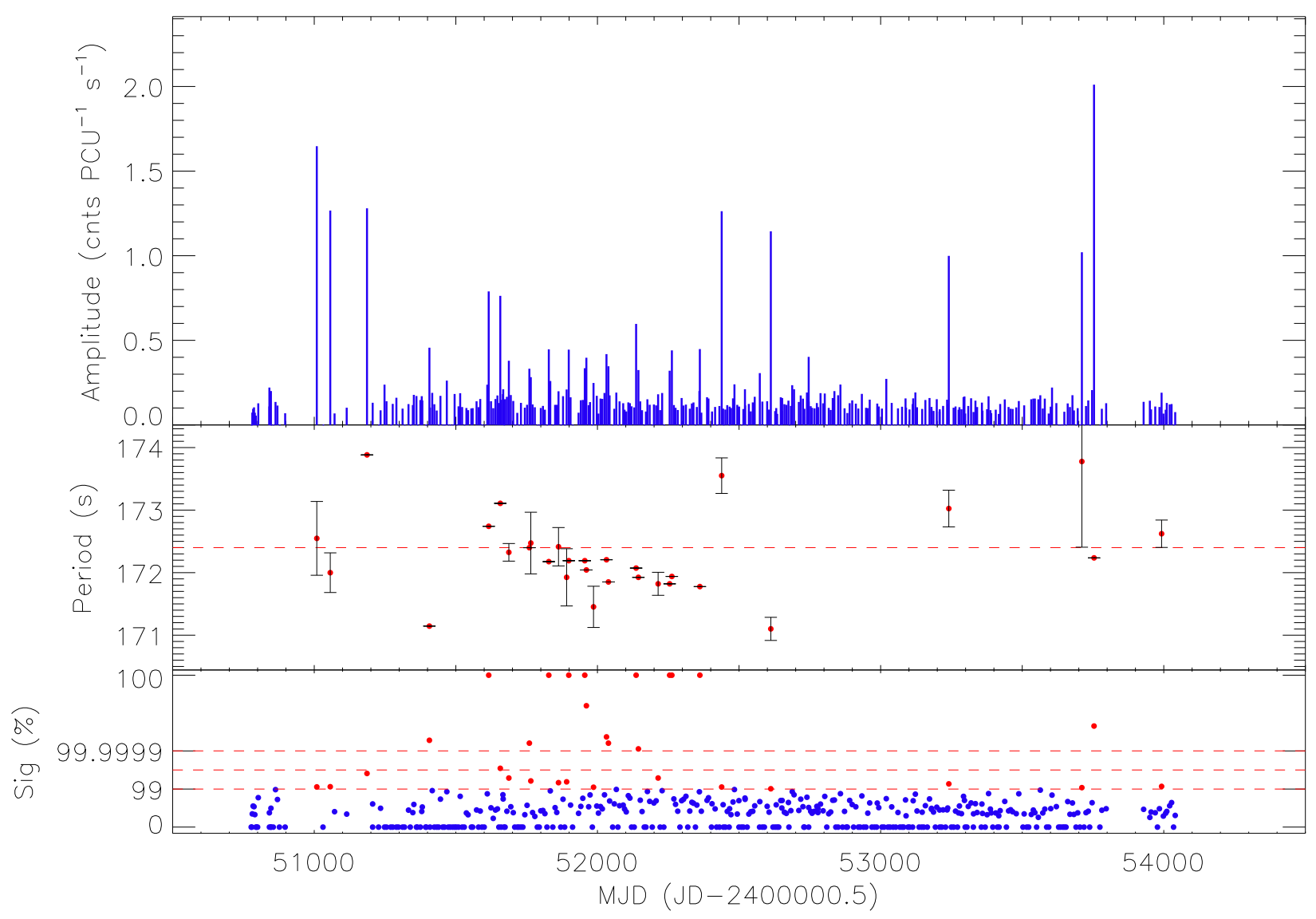

Fig. 30.- SXP172, X-ray amplitude light curve. 


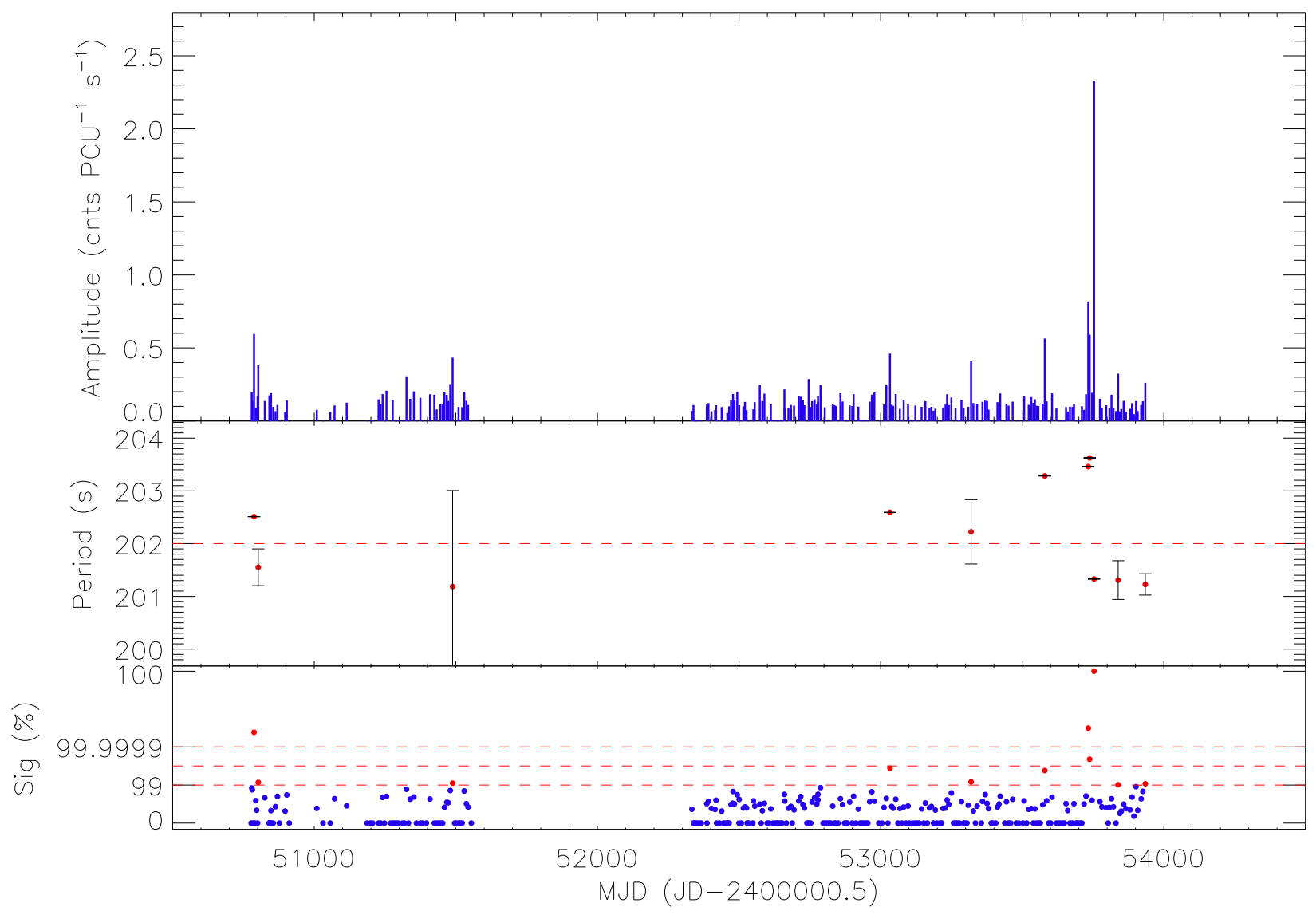

Fig. 31.- SXP202, X-ray amplitude light curve. 
panion as the emission line star [MA93] 172 and found an optical period of $48.8 \pm 0.6 \mathrm{~d}$, which they propose as the orbital period of the system. Further analysis of the OGLE light curve finds an ephemeris of MJD $50592 \pm 2+\mathrm{n} \times 49.2 \pm 0.2 \mathrm{~d}$ (Edge 2005d). Schmidtke \& Cowley (2005b) found a $49.1 \mathrm{~d}$ period in OGLE data.

Survey Results: Due to its location this pulsar was only observed consistently during AO5 and AO6 (see Fig. 32). No major outbursts were detected and the power spectrum shows no significant peak at the optical period, nor at any other. We note that the optical ephemeris does not agree with the X-ray activity.

\subsection{SXP280}

RX J0057.8-7202, AX J0058-72.0

\section{RA 005748.2 , dec -720240}

History: Discovered in March 1998 in an ASCA observation by Yokogawa \& Koyama (1998a) at a period of $280.4 \pm 0.3 \mathrm{~s}$. It is identified with the Be star [MA93] 1036, and Schmidtke et al. (2006) find a $127.3 \mathrm{~d}$ period in its OGLE data with an epoch of maximum brightness at MJD 52194.7. This pulsar was observed on 15 occasions by EINSTEIN, ROSAT and $A S C A$, but never detected above $L_{\mathrm{x}}=6.0 \times 10^{35} \mathrm{erg} \mathrm{s}^{-1}$ (Tsujimoto et al. 1999).

Survey Results: There are only 5 clear detections of this source throughout the survey, and the power spectrum shows no significant periods, although the highest peak is at $64.8 \mathrm{~d}$, which is $\sim \frac{1}{2}$ of the optical period. For this reason, the ephemeris lines and folded light curve are shown in Fig. 33. The tentative ephemeris of the X-ray period is MJD $52312 \pm 6$ $+\mathrm{n} \times 64.8 \pm 0.2 \mathrm{~d}$, which is within $\sim 12 \mathrm{~d}$ of the epoch of peak optical flux. A lack of earlier detections of this system in outburst makes it difficult to firmly establish an X-ray ephemeris.

\subsection{SXP293}

\section{XTE J0051-727}

\section{No position available}

History: Reported by Corbet et al. (2004c) from observations during this survey of the outburst at MJD 53097.

Survey Results: Only one of the 6 outbursts lasts longer than one week (Fig. 34(a)), which could imply this is a Type II outburst, and thus not expected to coincide with periastron passage. For this reason, it was removed from the data after initial timing analysis produced 


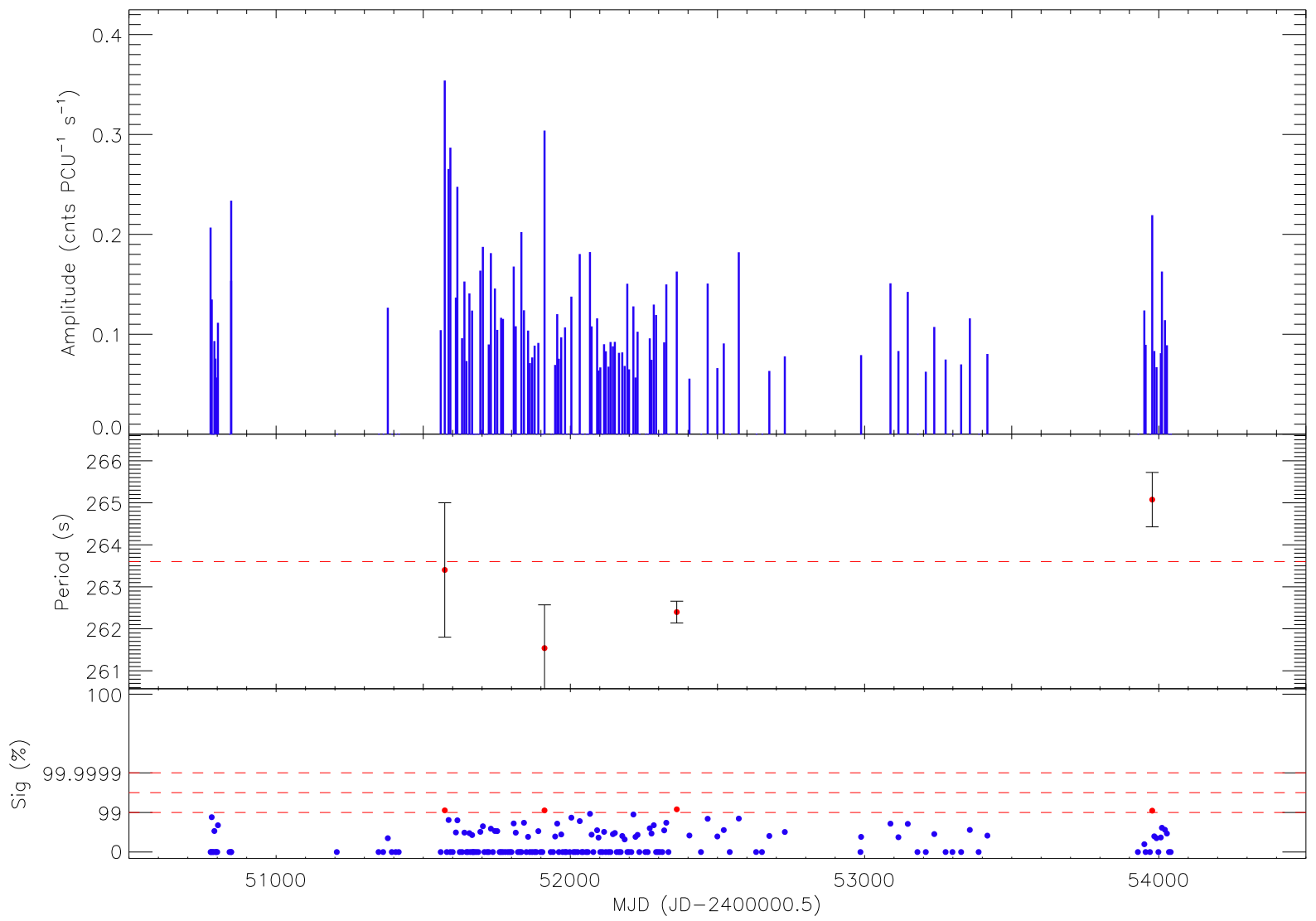

Fig. 32.- SXP264, X-ray amplitude light curve. 

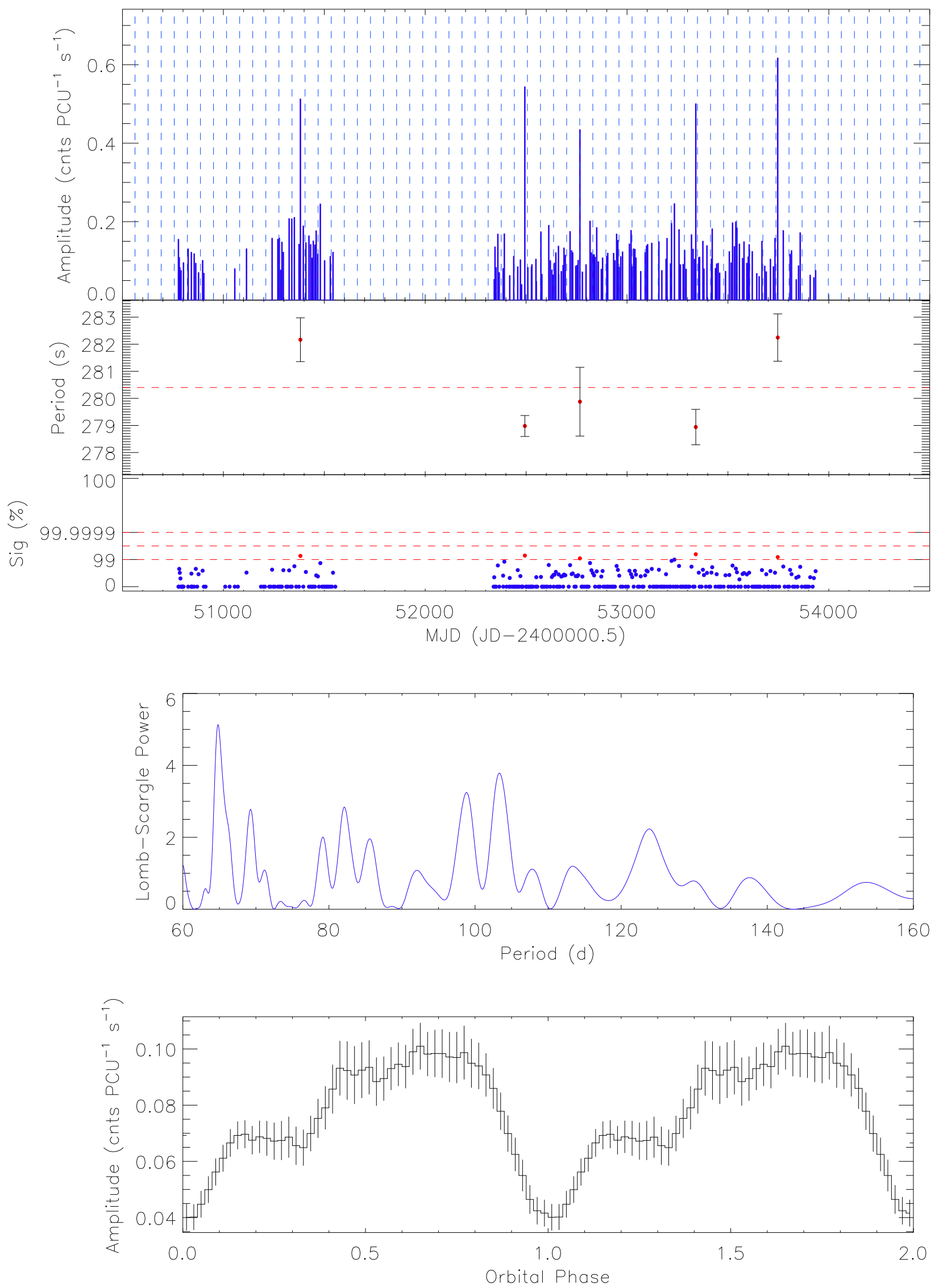

Fig. 33.- SXP280. a) Top: X-ray amplitude light curve. b) Middle: Lomb-Scargle power 
no results. The resulting power spectrum, while not showing any significant periods, does have its strongest peak at $151 \mathrm{~d}$, an ephemeris that agrees well with the remaining 5 detections. We suggest this is the likely orbital period of the system, with an ephemeris of MJD $52327.3 \pm 4.5+\mathrm{n} \times 151 \pm 1 \mathrm{~d}$.

\subsection{SXP304}

\section{RX J0101.0-7206, CXOU J010102.7-720658}

\section{RA 010101.7 , dec -720702}

History: Discovered in Chandra observations at $304.49 \pm 0.13 \mathrm{~s}$, the optical counterpart is identified as the emission line star [MA93] 1240 (Macomb et al. 2003). The authors measured an unusually high pulse fraction of $90 \pm 8 \%$ at a luminosity of $L_{\mathrm{x}}=1.1 \times 10^{34} \mathrm{erg} \mathrm{s}^{-1}$. Schmidtke \& Cowley (2006) suggest there may be a $520 \pm 12 \mathrm{~d}$ period in MACHO data of the optical counterpart [MA93] 1240.

Survey Results: This source was out of the field of view during AO5 and AO6, so it has only been adequately covered from AO7 onwards. A number of small outbursts were detected during MJD 52600 - 53000 (see Fig. 35), and it was not detected again until recently, in MJD 53747, when it began a $2-3$ week outburst, the longest and brightest observed so far in this survey. Lomb-Scargle analysis of the data outside of this outburst finds moderate power at $\sim 50 \mathrm{~d}$ and no significant power at the optical period. It should be noted that this source displays X-ray activity on timescales much shorter than the reported $520 \mathrm{~d}$ optical period.

\subsection{SXP323}

\section{RX J0050.7-7316, AX J0051-733}

\section{RA 005044.8 , dec -731606.0}

History: Pulsations at 323.2 $\pm 0.4 \mathrm{~s}$ were detected in November 1997 (Yokogawa \& Kovama 1998a) at the coordinates of the ROSAT source RX J0050.7-7316. Cowley et al. (1997) identified the optical counterpart as a Be star. This system has been found to exhibit optical and IR variability at periods of $\sim 0.7$ and $1.4 \mathrm{~d}$ (Coe et al. 2002) and $1.695 \mathrm{~d}$ (Coe et al. 2005). These periods are too short to be the orbital period of the system and are most likely nonradial pulsations in the Be star. Laycock et al. (2005) suggest an orbital period of $109 \pm 18 \mathrm{~d}$ from X-ray data earlier in this survey.

Survey Results: This pulsar showed quite regular, bright activity during the survey (see 

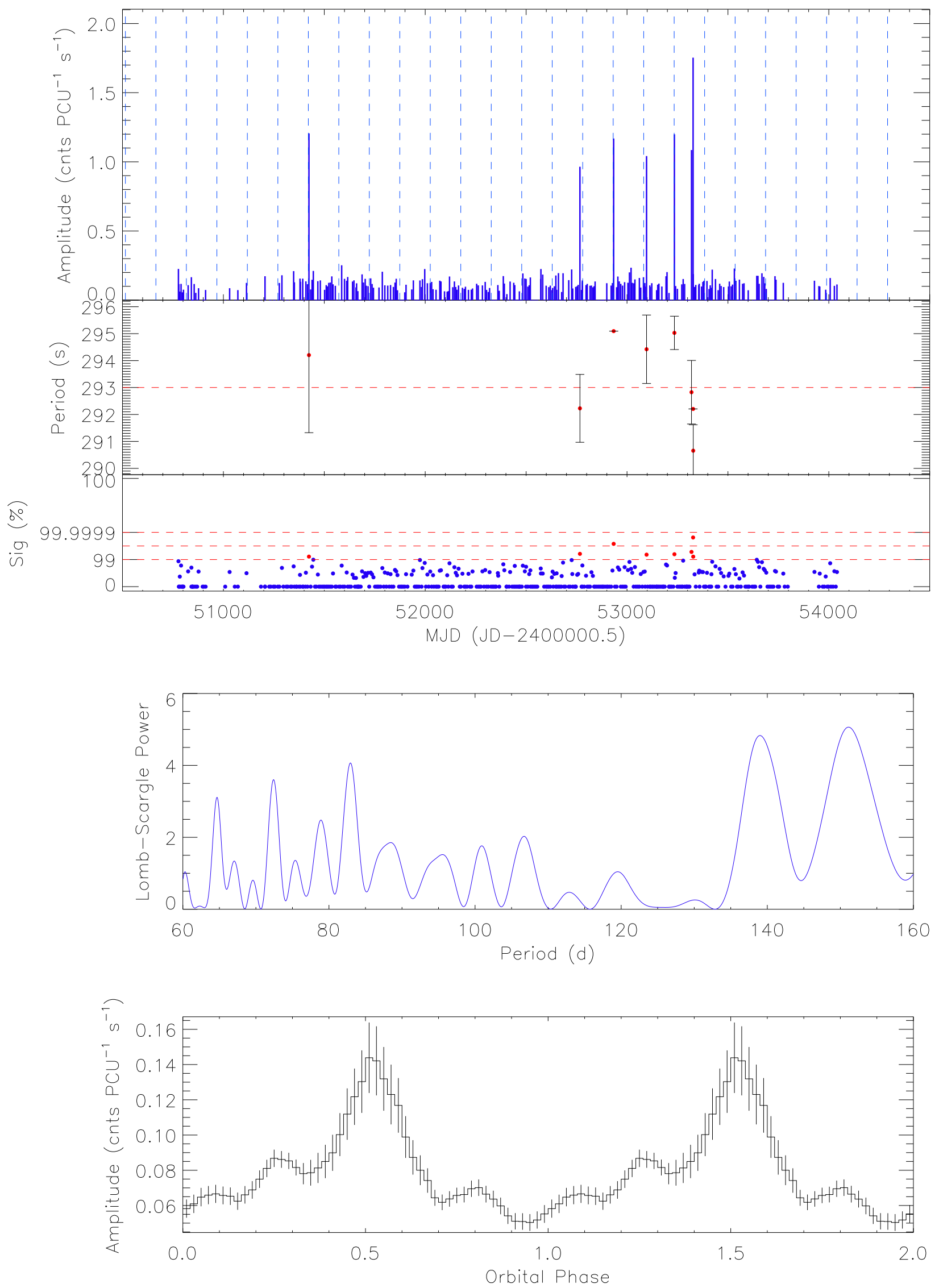

Fig. 34.- SXP293. a) Top: X-ray amplitude light curve. b) Middle: Lomb-Scargle power 


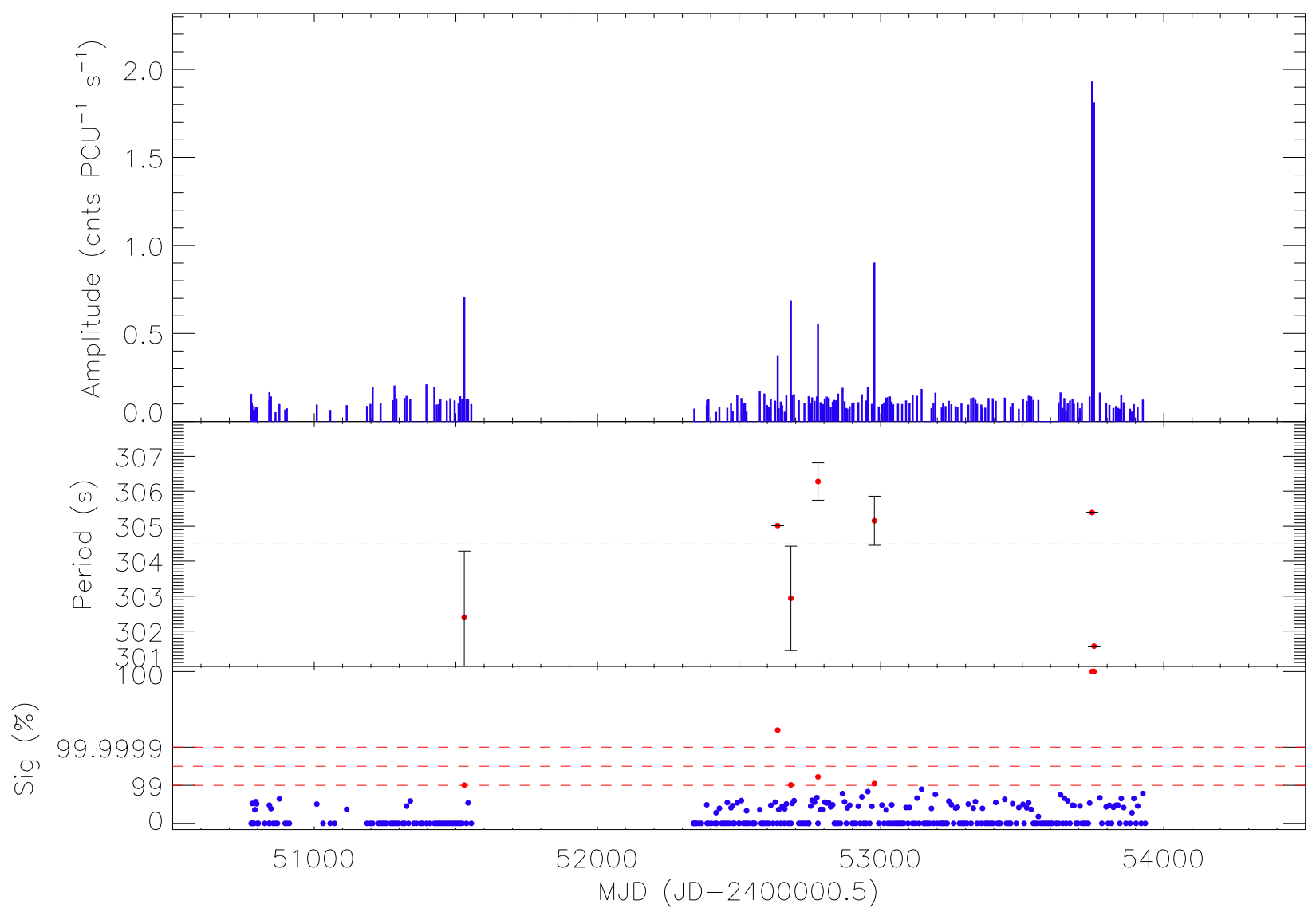

Fig. 35.- SXP304, X-ray amplitude light curve. 
Fig. 36(a)). We found that the outburst circa MJD 52960 skewed the timing analysis results, which we attribute to it being a Type II outburst; for this reason it was excluded from the analysis. The ephemeris found for the remaining outbursts is MJD $52336.9 \pm 3.5+$ $\mathrm{n} \times 116.6 \pm 0.6 \mathrm{~d}$, which is consistent with the orbital period proposed by Laycock et al. (2005).

\subsection{SXP348}

RX J0102-722, SAX J0103.2 -7209, AX J0103.2-7209

\section{RA 0103 13.0, dec -72 0918.0}

History: Pulsations at 345.2 $\pm 0.1 \mathrm{~s}$ were detected in BeppoSAX observations in July 1998 (Israel et al. 1998). Subsequently, pulsations at $348.9 \pm 0.1 \mathrm{~s}$ were found in archival $A S C A$ data from May 1996 implying a $\dot{P}=-5.39 \times 10^{-8} \mathrm{~s} \mathrm{~s}^{-1}$ (Yokogawa \& Koyama 1998b). The ROSAT source lies in a supernova remnant with a Be counterpart; a weak $93.9 \mathrm{~d}$ periodicity has been reported from OGLE data (Schmidtke \& Cowley 2006). Israel et al. (2000) suggest this is a persistent, low luminosity X-ray system.

Survey Results: Although this source has been detected by a number of different instruments, it was always at low luminosities $\left(\lesssim 10^{36} \mathrm{erg} \mathrm{s}^{-1}\right)$, so it is not surprising that there are not many detections in the survey Fig. 37. Furthermore, it has also been detected at a wider range of periods than other pulsars (from 340 to $348 \mathrm{~s}$ ), which makes it more difficult to pick out in the periodograms from weekly observations. Timing analysis reveals no significant periods.

\subsection{SXP452}

\section{RX J0101.3-7211}

\section{RA 0101 19.5, dec -72 1122}

History: Was initially proposed as an X-ray binary by Haberl et al. (2000). Pulsations were detected in XMM observations during 2001 at $455 \pm 2 \mathrm{~s}$ and in 1993 ROSAT data at 450-452 s (Sasaki et al. 2001), implying a $\dot{P}=2.3 \times 10^{-8} \mathrm{~s} \mathrm{~s}^{-1}$; the optical counterpart was identified as a Be star Sasaki et al. (2001). Schmidtke et al. (2004) propose an orbital period of $74.7 \mathrm{~d}$ for this system based on its optical variability.

Survey Results: With only 5 detections throughout the survey (Fig. 38), this source's periodogram shows no periodicities and there is no evidence for the reported $74.7 \mathrm{~d}$ optical 

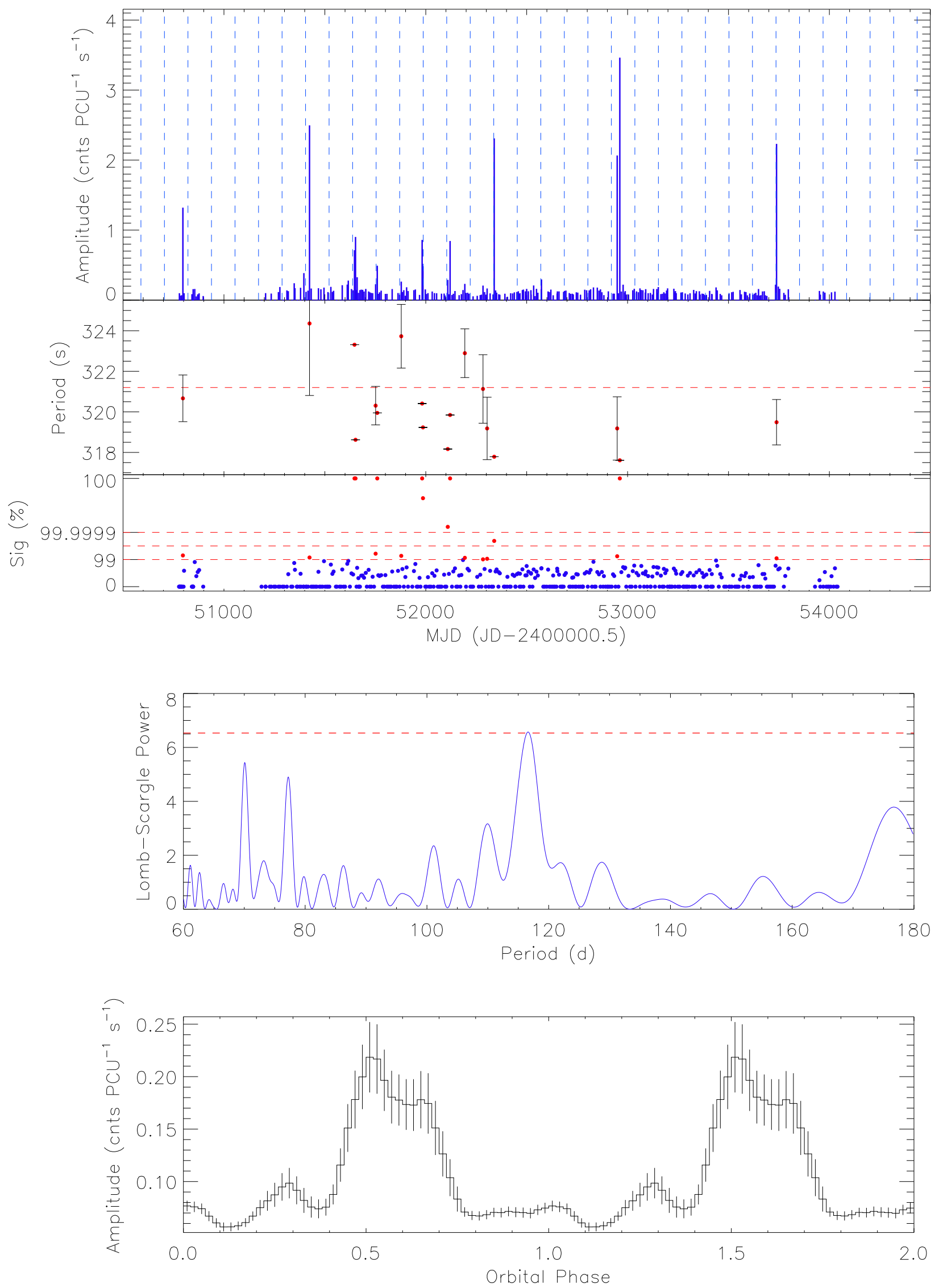

Fig. 36.- SXP323. a) Top: X-ray amplitude light curve. b) Middle: Lomb-Scargle power 


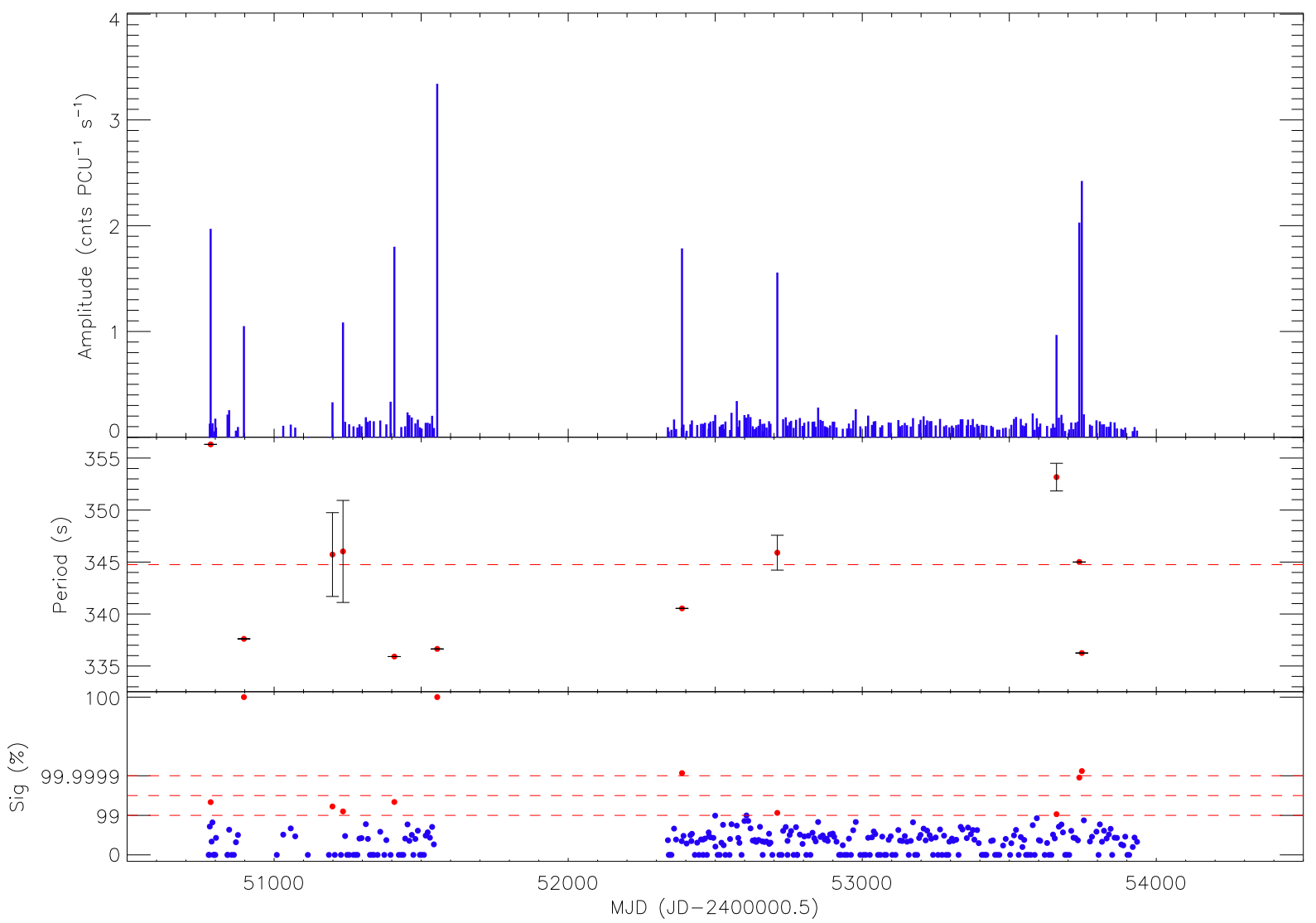

Fig. 37.- SXP348, X-ray amplitude light curve. 
period.

\subsection{SXP504}

RX J0054.9-7245, AX J0054.87244, CXOU J005455.6-724510 RA 005455.6 , dec -724510

History: Discovered in archival Chandra data with $503.5 \pm 6.7 \mathrm{~s}$ pulsations (Edge et al. 2004d.c). Also reported by Haberl et al. (2004) from an XMM observation at $499.2 \pm 0.7 \mathrm{~s}$ and $L_{\mathrm{x}}=4.3 \times 10^{35} \mathrm{erg} \mathrm{s}^{-1}$. An orbital period of $268.0 \pm 1.4 \mathrm{~d}$ was determined from optical (OGLE) data, and corroborated by preliminary X-ray data from the present survey (Edge et al. 2005b). The optical ephemeris was later refined to MJD 50556 $\pm 3+$ $\mathrm{n} \times 268.0 \pm 0.6 \mathrm{~d}$ (Edge et al. 2005c). Schmidtke \& Cowley (2005b) found a period of $273 \mathrm{~d}$ in OGLE data.

Survey Results: The light curve for this system can be seen in Fig. 39(a). Lomb-Scargle analysis of the entire survey (with or without the bright outbursts circa MJD 52440 and 52980) returns a slightly shorter orbital period to the optical one previously reported, but is consistent within errors. Furthermore, Edge et al. (2005c) find that the epochs of maximum X-ray flux are coincident with the optical outbursts. The ephemeris we find is MJD $52167.4 \pm 8.0+\mathrm{n} \times 265.3 \pm 2.9 \mathrm{~d}$. This source displays a lot of activity in between periastron passages, which might be indicative of a low eccentricity orbit and makes timing analysis more difficult.

\subsection{SXP565}

\section{CXOU J005736.2-721934}

\section{RA 005736.2 , dec -721934}

History: Discovered in Chandra observations at $564.81 \pm 0.41 \mathrm{~s}$ with a pulse fraction of $48 \pm 5 \%\left(L_{\mathrm{x}}=5.6 \times 10^{34} \mathrm{erg} \mathrm{s}^{-1}\right)$, the optical counterpart is idenified as the emission line star [MA93] 1020 (Macomb et al. 2003). An optical period of 95.3 d has been reported for this system (Schmidtke et al. 2004), but this period is not seen in OGLE data (Edge 2005d).

Survey Results: This source shows a lot of variability, but no clear outbursts (see Fig.40(a)). The power spectrum returns two significant peaks, with only the higher one providing a convincing orbital profile. The ephemeris for this period is MJD $52219.0 \pm 13.7+\mathrm{n} \times 151.8 \pm 1.0 \mathrm{~d}$. We note there is no power at the reported optical period nor does the optical ephemeris agree 


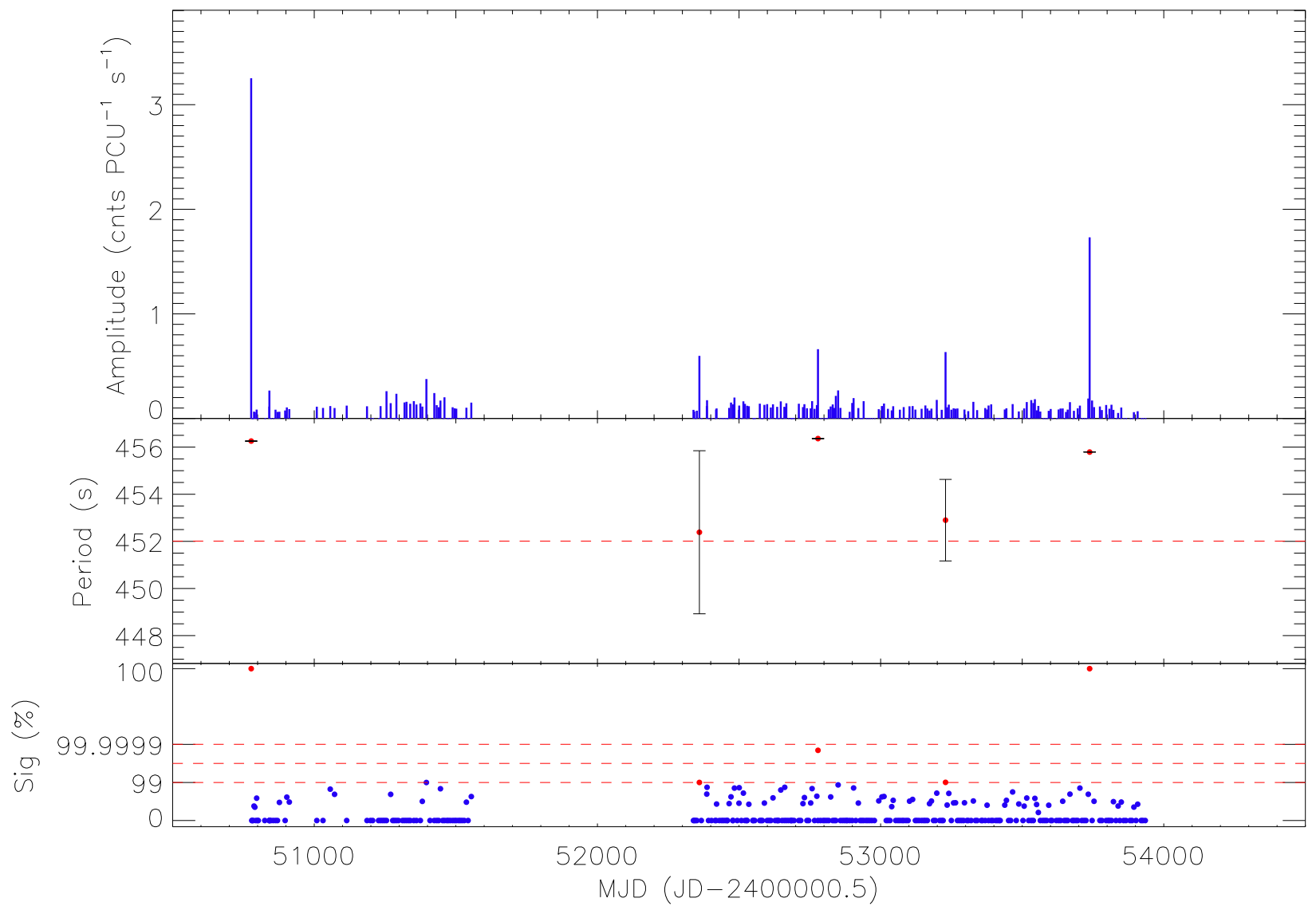

Fig. 38.- SXP452, X-ray amplitude light curve. 

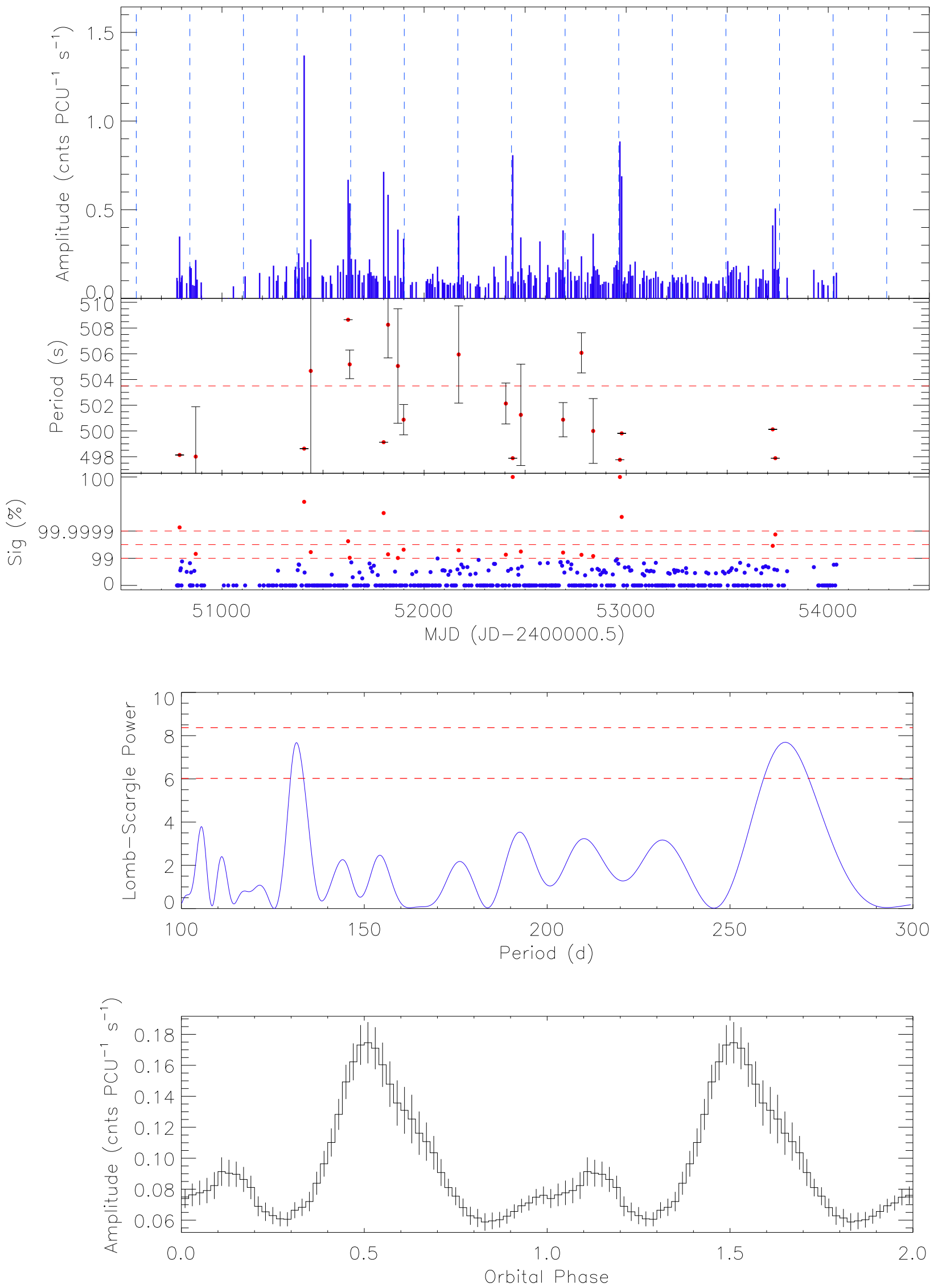

Fig. 39.- SXP504. a) Top: X-ray amplitude light curve. b) Middle: Lomb-Scargle power 
with the brightest X-ray detections of this source. In view of this, it is possible that the optical counterpart has been misidentified; however, there is no other bright candidate star within 10 arcsec of the very precise Chandra position. The folded light curve has a unique shape, showing significant activity around periastron. The asymmetry of the periastron peak is likely due to the presence of an accretion disk around the neutron star that is not completely consumed by the time it reaches apastron, where its lower orbital velocity allows it to "top up" its disk from the Be star's wind.

\subsection{SXP700}

\section{CXOU J010206.6-714115}

\section{RA 0102 06.69, dec -714115.8}

History: Discovered as part of the aforementioned Chandra survey of the SMC wing reported in McGowan et al. (2007). They detected a source with $L_{\mathrm{x}}=6.0 \times 10^{35} \mathrm{erg} \mathrm{s}^{-1}$ (35 $\pm 5 \%$ pulse fraction) with pulsations at $700.54 \pm 34.53 \mathrm{~s}$ and found its position to coincide with the emission line star [MA93] 1301, a $V=14.6$ O9 star. The OGLE data available for this object show a periodic modulation at 267.38 $\pm 15.10 \mathrm{~d}$ (McGowan et al. 2007).

Survey Results: Due to its proximity in period to SXP701, limitations of the current analysis script (which cannot work on two pulsars of such similar spin periods) have not allowed the extraction of an X-ray light curve for this pulsar. However, it has not contaminated the data presented for SXP701 because, even though they are $\sim 66-$ arcmin away from each other, they were never within the same field of view.

\subsection{SXP701}

\section{RX J0055.2-7238, XMMU J005517.9-723853}

\section{RA 005517.9 , dec -723853}

History: First observed during an XMM TOO observation at $701.6 \pm 1.4 \mathrm{~s}$ and located within the error circle of a ROSAT object (Haberl et al. 2004). Fabrycky (2005) finds optical periods of 6.832 and $15.587 \mathrm{~h}$, which are attributed to stellar pulsations. A weak $412 \mathrm{~d}$ period has been seen in MACHO data (Schmidtke \& Cowley 2005b).

Survey Results: Similar to SXP565, it displays much X-ray variability with no bright outbursts (see Fig. 41). Unfortunately, timing analysis provides no clear periodicities. 

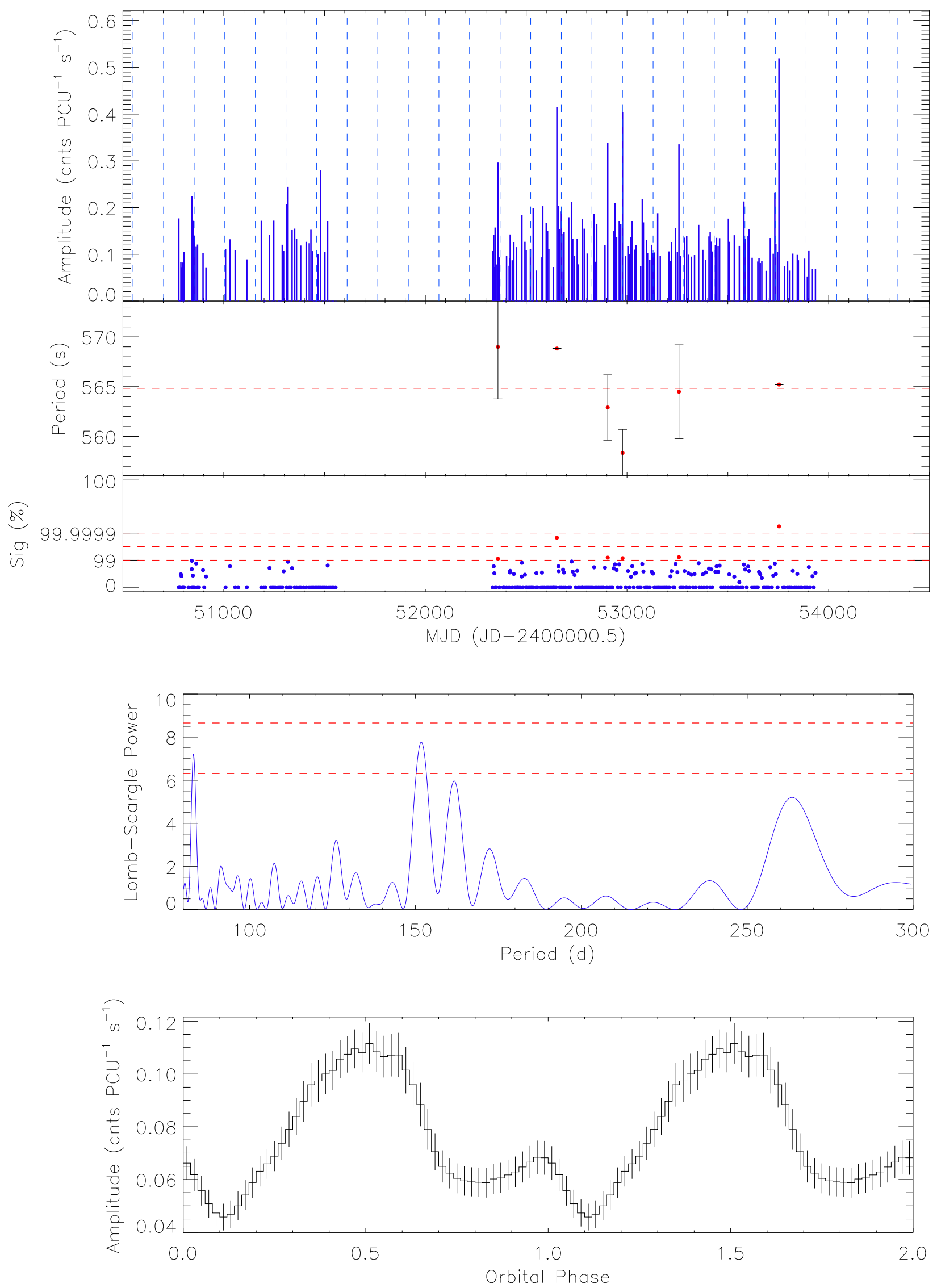

Fig. 40.- SXP565. a) Top: X-ray amplitude light curve. b) Middle: Lomb-Scargle power 


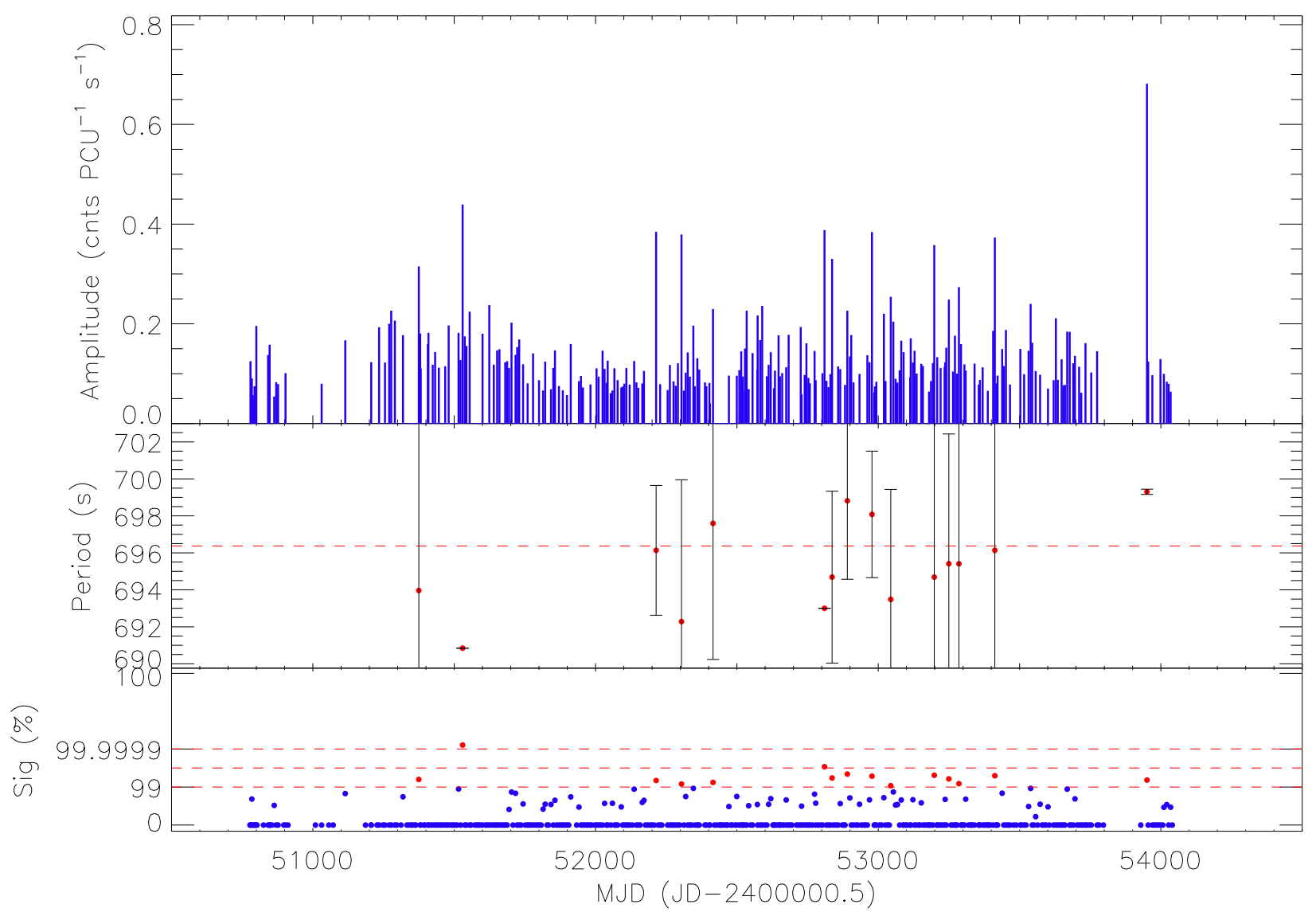

Fig. 41.- SXP701, X-ray amplitude light curve. 


\subsection{SXP756}

RX J0049.6-7323, AX J0049.4-7323

RA 004942.42 , dec -732315.9

History: Pulsations at $755.5 \pm 0.6 \mathrm{~s}$ were detected in a very long ( $\sim 177 \mathrm{ks}) A S C A$ observation of the SMC in April 2000, the source was detected at a luminosity of $L_{\mathrm{x}}=5 \times 10^{35} \mathrm{erg} \mathrm{s}^{-1}$ (Yokogawa et al. 2000b). The optical counterpart was later established as a $\mathrm{V}=15 \mathrm{Be}$ star (Edge \& Coe 2003). Lavcock (2002) and Lavcock et al. (2005) find an X-ray period of $396 \pm 5$ d while Cowley \& Schmidtke (2003); Schmidtke et al. (2004) report recurring outbursts at $\sim 394 \mathrm{~d}$ intervals in $R$ and $V$ MACHO data.

Survey Results: Coverage of this pulsar has been sparse due to its southern location in the SMC, only visible to observations of Positions 4 and 5. Despite this, it has been detected various times in outburst, the brightest of which was a 3 week outburst (Fig. 42(a)). We decided to remove the brightest point from this outburst so as not to skew the Lomb-Scargle power spectrum. A strong period is found at $194.9 \mathrm{~d}$ which we believe to be the first harmonic of the orbital period. Looking at the light curve it is evident that the bright detections are spaced $\sim 400 \mathrm{~d}$ apart 3 . Moreover, this would be in agreement with the optical period of the system, so we use twice the value of the harmonic as the orbital period and derive the ephemeris MJD $52196.1 \pm 3.9+\mathrm{n} \times 389.9 \pm 7.0 \mathrm{~d}$. This ephemeris places the first outburst in the data at MJD 51416, which is consistent with the last optical outburst available in the MACHO data (Cowley \& Schmidtke 2003).

\subsection{SXP1323}

RX J0103.6-7201

RA 010337.5 , dec -720133.2

History: This is the source with the longest known pulse period in the SMC, SXP1323 was reported by Haberl \& Pietsch (2005) in a number of archival XMM observations. The authors identify the emission line star [MA93] 1393 ( $\mathrm{V} \simeq 14.6)$ as the optical counterpart. Schmidtke \& Cowley (2006) report three strong periods for this source from OGLE II data: $0.41 \mathrm{~d}, 0.88 \mathrm{~d}$ and $26.16 \mathrm{~d}$; they attribute the first two to non-radial pulsations of the Be star but suggest the latter might be the orbital period.

\footnotetext{
${ }^{3}$ Also note that only one peak appears in the orbital profile in Fig. 42(b). If $194.9 \mathrm{~d}$ were the actual orbital period, the plot would show two peaks per orbital cycle.
} 

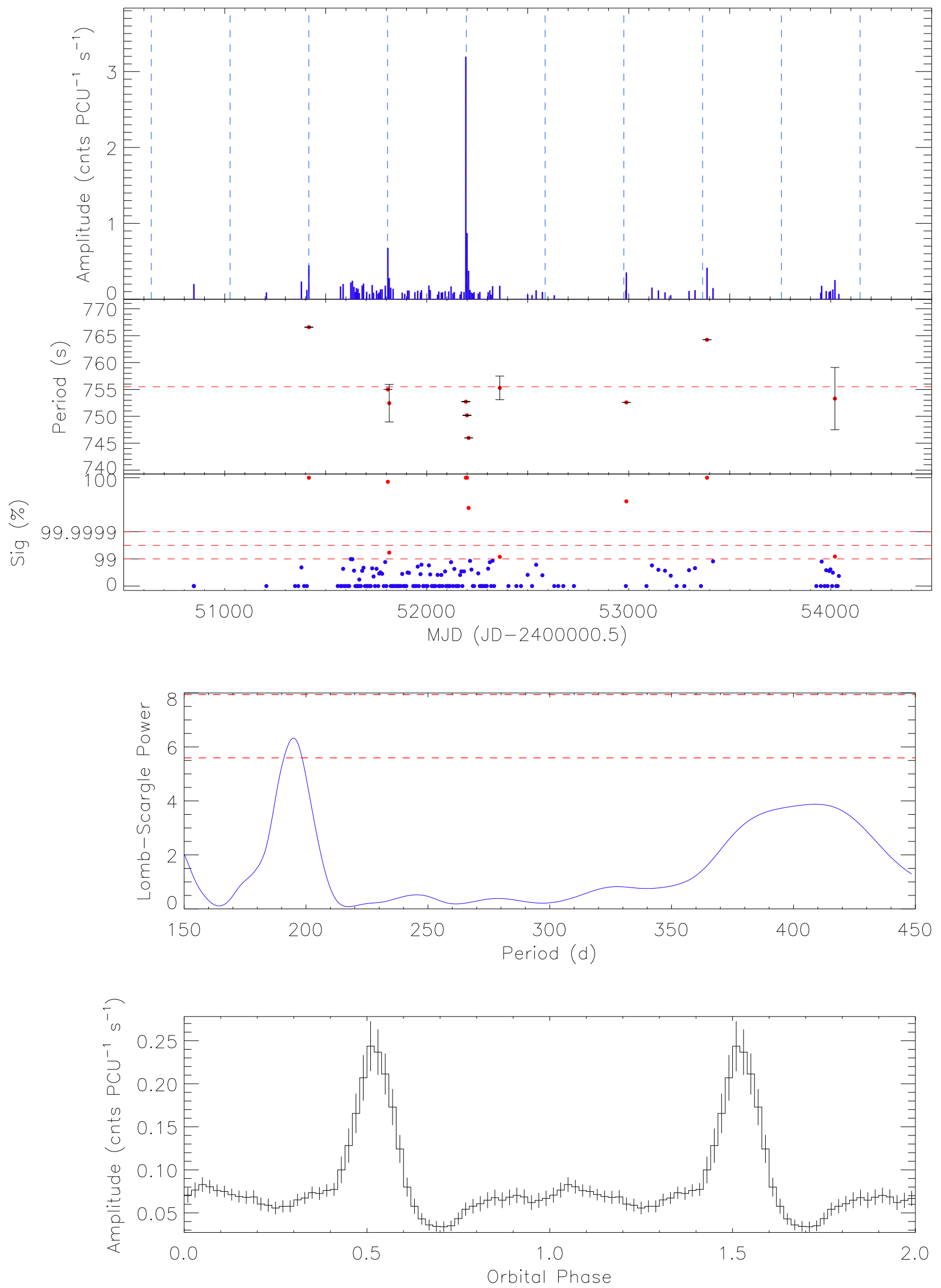

Fig. 42.-SXP756. a) Top: X-ray amplitude light curve. b) Middle: Lomb-Scargle power 
Survey Results: This source is difficult to detect due to its long period (requiring observations with a baseline longer than $\sim 4 \mathrm{ks}$ ) and its location near the edge of Position 1/A. Despite these limitations, a number of bright outbursts have been detected but no periodicities can be found in the sparse data (see Fig. 43).

\section{Discussion}

Table 3 presents a summary of the timing analysis results for the SMC pulsars, providing orbital periods and ephemerides where available, together with periods observed at other wavelengths. In cases when there is only weak evidence for an orbital period in the X-ray data, the number is given in parentheses.

Those systems with unequivocal orbital periods have allowed us to study and compare a selection of well-defined orbital profiles. In particular, we consider the profiles of the following systems that have shown regular activity at, or around, periastron pasage: SXP6.85, SXP7.78, SXP46.6, SXP59.0, SXP82.4, SXP144, SXP169 and SXP756. It is notable that their profiles all share a similar shape, even though their orbital periods cover a wide range (from $\sim 45-390 \mathrm{~d}$ ): they are relatively narrow (except for SXP756, all widths are between 0.40 and 0.55 wide in orbital phase), and they are very symmetrical in shape (suggesting accretion takes place fluidly and increases smoothly as the neutron star approaches the deccretion disk, to decrease in an equally smooth manner after periastron). Other systems display different characteristics, some show sharp rises with long declines (e.g.,SXP323, Fig. 36(b)), the opposite (e.g., SXP16.6, Fig. 12(b)), or even long, plateau-like profiles (e.g.,SXP280, Fig. 33(b)).

A number of profiles show a small increase in flux half an orbital phase away from the maximum (e.g., SXP7.78, SXP89.0, SXP144, SXP504 and SXP565), suggesting that accretion is taking place on a smaller scale, possibly from the Be star's wind, when the neutron star is travelling slowly enough to accrete from it. Given the range of orbit sizes in the systems that exhibit this behaviour (judging from the orbital periods), it may be the eccentricity of the orbit that determines whether apastron accretion takes place (because the more eccentric the orbit, the slower the neutron star will be travelling at apastron).

Throughout the length of the survey there have been some pulsars whose spin periods have undergone slow, persistent evolution, e.g., SXP7.78 (spin down), SXP46.6 (spin up), SXP59.0 (spin up) or SXP144 (spin down), while others have changed their pulse period over the course of a few weeks, e.g., SXP15.3 (spin up). And still, some other systems have not changed their spin periods significantly despite bright and/or persistent activity, e.g., 


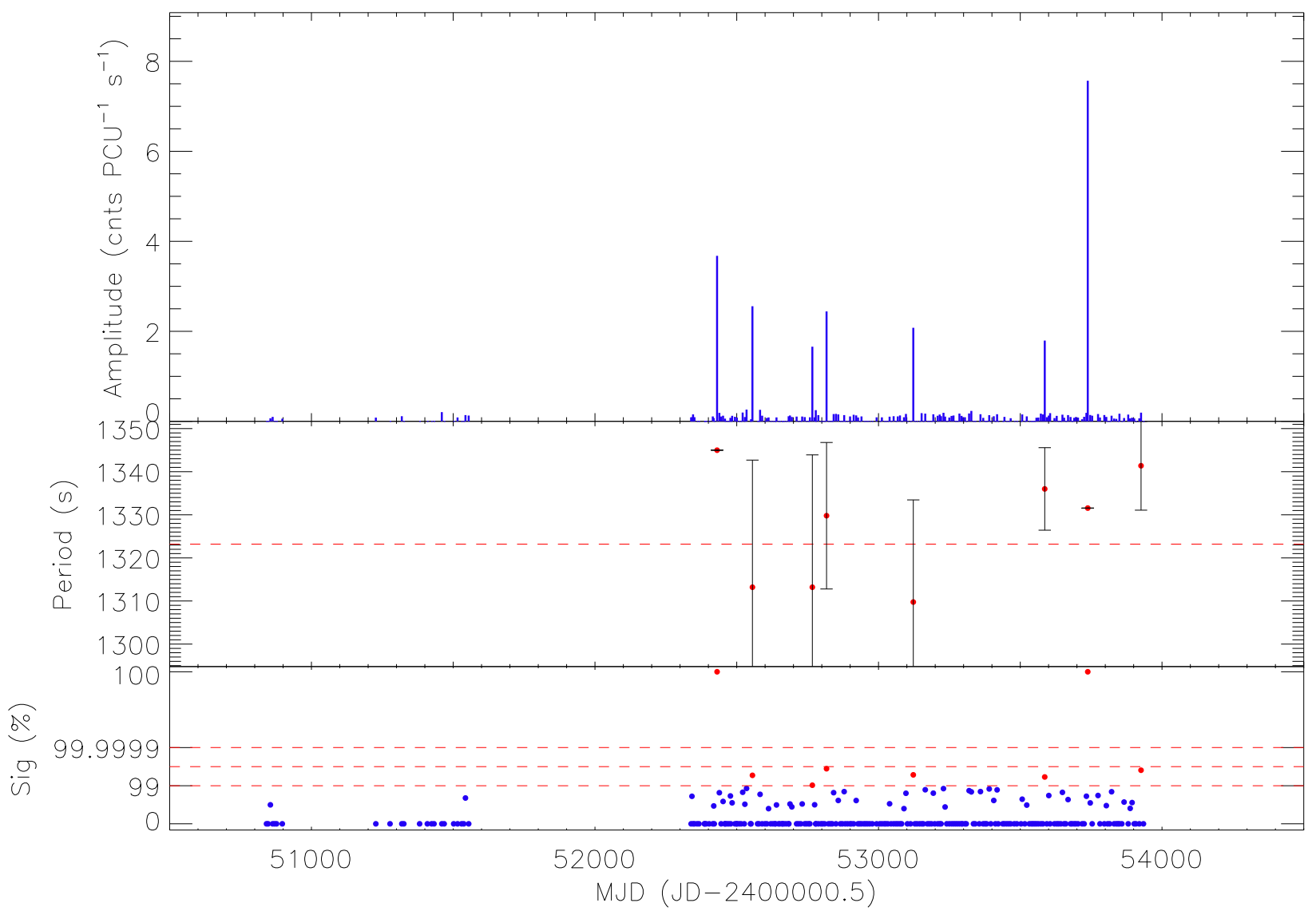

Fig. 43.- SXP1323, X-ray amplitude light curve. 
SXP2.37, SXP4.78 or SXP8.88. This suggests there are a number of pulsars that must be rotating close to, or at, their equilibrium spin periods, as suggested by Corbet (1984). A lack of modulation in the spin period throughout a long outburst (as in SXP18.3's outburst circa MJD 54000) further reveals systems that are likely being observed at a low inclination (face on). Unfortunately, despite the number of orbital periods known, it has still not been possible to calculate the complete set of orbital parameters of any SMC system (with the exception of SXP0.92 (Kaspi et al. 1993)), so orbital inclination angles and their effects on pulse arrival times remain a matter of speculation.

An interesting case is presented by SXP348; Israel et al. (2000) suggest this is a persistent, low luminosity system, and recent Chandra observations (McGowan et al. 2007) tend to support this notion after detecting the pulsar at its shortest recorded pulse period until then, 337.5s. Given that it was observed in 1996 at $349 \mathrm{~s}$ in $A S C A$ data, we can deduce that it has spun down $11.5 \mathrm{~s}$ in $\sim 10$ years, implying a $\dot{P}=3.6 \times 10^{-8} \mathrm{~s} \mathrm{~s}^{-1}$. An estimate for the accretion necessary to produce the required torque on this timescale implies a timeaveraged X-ray luminosity of just $L_{\mathrm{x}} \geq 3.3 \times 10^{35} \mathrm{erg} \mathrm{s}^{-1}$, which would explain why it was rarely detected during the present survey. However, RXTE detected it a number of times at longer pulse periods than would have been expected; a clear occasion was during the short outburst circa MJD 53740, when the system was detected at a high significance on 2 consecutive weeks with periods of $345.0 \mathrm{~s}$ and $336.2 \mathrm{~s}$, respectively. If this spin-up were all intrinsic, the expected luminosity would be $L_{\mathrm{x}} \geq 1.1 \times 10^{38} \mathrm{erg} \mathrm{s}^{-1}$, which is not inconceivable for a Type II outburst. McGowan et al. (2007) detected it $30 \mathrm{~d}$ later (MJD 53777) at $L_{\mathrm{x}}=4.2 \times 10^{37} \mathrm{erg} \mathrm{s}^{-1}$, but $R X T E$ did not detect it 2 days prior to that (MJD 53774). So what is happening in this system? It is not clear whether we are detecting orbitally-induced Doppler-shifted pulse periods or if SXP348 is actually undergoing massive spin up every time it outbursts. Given the pulse periods of the detections in Fig. 37, it is unclear whether the pulsar is, in fact, spinning down (as proposed by Israel et al. (2000)); it could very well be spinning up.

Another case involving changes in spin is that of SXP59.0 (Fig. 17(a)). It went undetected by RXTE during MJD 51550-52500, during which time its pulse period increased $\sim 0.45 \mathrm{~s}$. If this spin-down were a product of reverse accretion torques, we would have expected to see outbursts at $L_{\mathrm{x}} \geq 2.6 \times 10^{36} \mathrm{erg} \mathrm{s}^{-1}$, comparable in strength with those detected during the survey. Although SXP59.0 was not close to the center of the field of view during the spin-down period (as it was for most of the rest of the survey), it should still have been detectable. This suggests that SXP59.0 was spun down through mechanisms other than reverse accretion torques, possibly through the propeller effect.

Yet another example of strange spin behaviour is that of SXP144. Given its pulse period, 
and based on the Corbet diagram, its orbital period should be $\sim 90 \mathrm{~d}$; so with its clear $59.4 \mathrm{~d}$ orbital period, the expectation is that it would spin up during outburst. However, quite the opposite is what RXTE has detected. This system went into outburst at least once every orbit over a period of $\sim 1160 \mathrm{~d}$, during which time it spun down $\sim 1.7 \mathrm{~s}$. It would seem that factors other than just the orbital and spin periods govern the behaviour and evolution of these binary systems, and they need to be taken into account. Orbital eccentricity and/or the magnetic field strength of the pulsar are likely to be important factors in determining the relationship between the spin and orbital periods.

\section{Conclusion}

We have presented the most comprehensive colection of pulsed light curves for 41 probable Be/X-ray systems in the SMC and have determined an X-ray (likely orbital) ephemeris for 21 of them while presenting possible periods for 6 others. 10 of these ephemerides are from new X-ray periods, while 6 others are improvements of previously known ephemerides. Of the systems exhibiting periodicities in the X-ray, 19 also show optical periodicities, although it is noteworthy that the optical and X-ray periods only agree on 7 occasions. Of the remaining 12,3 show an optical period that is half the X-ray period while 1 exhibits an optical modulation that is twice the X-ray period. However, there are still 11 systems with a known optical period that show no modulation in X-rays. The systems exhibiting a large discrepancy between the optical and X-ray periods suggest the possibility that the optical counterpart to the X-ray system has been missidentified, or that the interaction between the Be star's disk and the neutron star is more complicated than originally thought, although optical periods can be affected by pulsations of the primary star, and X-ray periods by Type II outbursts. Nonetheless, for extragalactic X-ray binaries, long-term monitoring has proven to be the most successful method of study. 
Table 3. X-ray binary systems in the SMC

\begin{tabular}{|c|c|c|c|c|c|c|}
\hline Object & $P_{\mathrm{s}}(\mathrm{s})$ & RA & dec & X-ray $T_{\mathrm{P}}(\mathrm{MJD})$ & X-ray $P_{\text {orb }}$ (days) & Other $P_{\text {orb }}($ days $)$ \\
\hline SXP0.09 & 0.087 & 004235.0 & -734030.0 & - & - & - \\
\hline SXP0.72 & 0.716 & 011705.2 & -732636.0 & - & - & - \\
\hline SXP0.92 & 0.92 & 004535.0 & -731902.0 & - & - & $51^{\mathrm{a}}$ \\
\hline SXP2.16 & 2.165 & 011900 & -731227 & - & - & - \\
\hline SXP2.37 & 2.374 & 005434.0 & -734103.0 & - & - & - \\
\hline SXP2.76 & 2.763 & 005911.7 & -713848.0 & - & - & $82.1^{\mathrm{b}}$ \\
\hline SXP3.34 & 3.34 & 010502.0 & -721100.0 & - & - & - \\
\hline SXP4.78 & 4.782 & 005206.6 & -722044 & - & $(34.1)$ & $23.9^{\mathrm{c}}$ \\
\hline SXP6.85 & 6.848 & 010253.1 & -724433 & $52318.5 \pm 7.9$ & $112.5 \pm 0.5$ & $24.82^{\mathrm{d}}$ \\
\hline SXP7.78 & 7.780 & 005206 & -722606 & $52250.9 \pm 1.4$ & $44.92 \pm 0.06$ & $44.86^{\mathrm{e}}, 44.6^{\mathrm{f}}, 44.8^{\mathrm{g}}$ \\
\hline SXP8.02 & 8.020 & 010041.8 & -721136 & - & - & - \\
\hline SXP8.88 & 8.880 & 005152.0 & -723151.7 & $52392.2 \pm 0.9$ & $28.47 \pm 0.04$ & $33.4^{\mathrm{h}}$ \\
\hline SXP9.13 & 9.130 & 004913.6 & -731139 & $52380.5 \pm 2.3$ & $77.2 \pm 0.3$ & $40.17^{\mathrm{g}}, 91.5^{\mathrm{i}}$ \\
\hline SXP15.3 & 15.30 & 005214 & -73 1919 & - & $(28)$ & $75.1^{\mathrm{j}}$ \\
\hline SXP16.6 & 16.52 & - & - & $52373.5 \pm 1.0$ & $33.72 \pm 0.05$ & - \\
\hline SXP18.3 & 18.37 & 0050 & -7242 & $52275.6 \pm 0.9$ & $17.73 \pm 0.01$ & $34.6^{\mathrm{k}}$ \\
\hline SXP22.1 & 22.07 & 011740.5 & -733052.0 & - & - & - \\
\hline SXP31.0 & 31.01 & 011109.0 & -731646.0 & - & - & $90.4^{\mathrm{b}}$ \\
\hline SXP34.1 & 34.08 & 005526.9 & $-72 \quad 1059.9$ & - & - & - \\
\hline SXP46.6 & 46.59 & 005358.5 & -722635.0 & $52293.9 \pm 1.4$ & $137.36 \pm 0.35$ & $69.2^{1}, 138.4^{1}$ \\
\hline SXP51.0 & 51.00 & - & - & - & - & - \\
\hline SXP59.0 & 58.86 & 005456.6 & -722650 & $52306.1 \pm 3.7$ & $122.10 \pm 0.38$ & $60.2^{\mathrm{m}}$ \\
\hline SXP65.8 & 65.78 & 010712.63 & -723533.8 & - & - & $110.6^{\mathrm{n}}$ \\
\hline SXP74.7 & 74.70 & 004904 & -725054 & $52319.0 \pm 3.1$ & $61.6 \pm 0.2$ & $33.4^{\mathrm{o}}$ \\
\hline SXP82.4 & 82.40 & 005209 & -723803 & $52089.0 \pm 3.6$ & $362.3 \pm 4.1$ & $\sim 380^{\mathrm{P}}$ \\
\hline SXP89.0 & 89.00 & - & - & $52337.5 \pm 6.1$ & $87.6 \pm 0.3$ & - \\
\hline SXP91.1 & 91.10 & 005055 & -721338 & $52197.9 \pm 8.2$ & $117.8 \pm 0.5$ & $88.25^{\mathrm{q}}$ \\
\hline SXP95.2 & 95.20 & 005200 & -724500 & - & $(71.3)$ & - \\
\hline SXP101 & 101.40 & 005726.8 & -732502 & - & $(25.2)$ & $21.9^{\mathrm{r}}$ \\
\hline SXP138 & 138.00 & 005323.8 & -722715.0 & $52400.5 \pm 5.2$ & $103.6 \pm 0.5$ & $\sim 125^{\mathrm{s}}, 122-123^{\mathrm{t}}$ \\
\hline SXP140 & 140.99 & 005605.7 & -722200.0 & - & - & $197^{\mathrm{t}}$ \\
\hline SXP144 & 144.00 & - & - & $52368.9 \pm 1.8$ & $59.38 \pm 0.09$ & - \\
\hline SXP152 & 152.10 & 005749 & -720759 & - & - & - \\
\hline SXP169 & 167.35 & 005254.0 & -715808.0 & $52240.1 \pm 2.1$ & $68.54 \pm 0.15$ & $67.6^{\mathrm{u}}$ \\
\hline SXP172 & 172.40 & 005152 & -731035 & - & $(70)$ & $69.9^{\mathrm{v}}$ \\
\hline SXP202 & 202.00 & 005920.8 & -722317 & - & $(91)$ & - \\
\hline SXP264 & 263.60 & 004723.7 & -731225 & - & - & $49.2^{\mathrm{g}}, 49.1^{\mathrm{w}}$ \\
\hline SXP280 & 280.40 & 005748.2 & -720240 & $52312 \pm 6$ & $64.8 \pm 0.2$ & $127.3^{\mathrm{u}}$ \\
\hline SXP293 & 293.00 & - & - & $52327.3 \pm 4.5$ & $151 \pm 1$ & - \\
\hline
\end{tabular}


Table 3-Continued

\begin{tabular}{ccccccc}
\hline \hline Object & $P_{\mathrm{s}}(\mathrm{s})$ & $\mathrm{RA}$ & $\mathrm{dec}$ & X-ray $T_{\mathrm{P}}(\mathrm{MJD})$ & X-ray $P_{\text {orb }}($ days $)$ & Other $P_{\text {orb }}($ days $)$ \\
\hline SXP304 & 304.49 & 010101.7 & -720702 & - & - & $520^{\mathrm{t}}$ \\
SXP323 & 321.20 & 005044.8 & -731606.0 & $52336.9 \pm 3.5$ & $116.6 \pm 0.6$ & - \\
SXP348 & 344.75 & 010313.0 & -720918.0 & - & - & $93.9^{\mathrm{v}}$ \\
SXP452 & 452.01 & 010119.5 & -721122 & - & - & $74.7^{\mathrm{x}}$ \\
SXP504 & 503.50 & 005455.6 & -724510.0 & $52167.4 \pm 8.0$ & $265.3 \pm 2.9$ & $268.0^{\mathrm{y}}, 273^{\mathrm{w}}$ \\
SXP565 & 564.83 & 005736.2 & -721934.0 & $52219.0 \pm 13.7$ & $151.8 \pm 1.0$ & $95.3^{\mathrm{q}}$ \\
SXP700 & 700.5 & 010206.69 & -714115.8 & - & - & $267^{\mathrm{z}}$ \\
SXP701 & 696.37 & 005517.9 & -723853.0 & - & - & $412^{\mathrm{aa}}$ \\
SXP756 & 755.50 & 004942.42 & -732315.9 & $52196.1 \pm 3.9$ & $389.9 \pm 7.0$ & $394^{\mathrm{ab}}$ \\
SXP1323 & 1323.20 & 010337.5 & -720133.2 & - & - & $26.16^{\mathrm{v}}$ \\
\hline
\end{tabular}

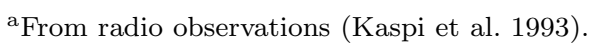

${ }^{\mathrm{b}}$ From OGLE III data (Schmidtke et al. 2006).

${ }^{\mathrm{c}}$ From MACHO data of the possible optical counterpart (Coe et al. 2005).

${ }^{\mathrm{d}}$ From OGLE and MACHO data (Schmidtke \& Cowley 2007).

${ }^{\mathrm{e}}$ From MACHO data (Cowley \& Schmidtke 2004).

${ }^{\mathrm{f}}$ From MACHO data (Coe et al. 2005).

gFrom OGLE data (Edge 2005d).

${ }^{\mathrm{h}}$ From OGLE and MACHO data (Schmidtke \& Cowlev 2006).

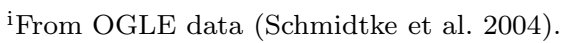

${ }^{\mathrm{j}}$ From OGLE and MACHO data (Edge 2005d).

${ }^{\mathrm{k}}$ From $R X T E$ data (Corbet et al. 2004d).

${ }^{1}$ From OGLE data (Schmidtke et al. 2007).

${ }^{\mathrm{m}}$ From OGLE and MACHO data (Schmidtke \& Cowlev 2005b).

${ }^{\mathrm{n}}$ From MACHO data (Schmidtke \& Cowley 2007b).

${ }^{\circ}$ From OGLE data (Schmidtke \& Cowlev 2005b; Edge 2005d).

PFrom OGLE data (Edge 2006, private communication).

${ }^{\mathrm{q}}$ From MACHO data (Schmidtke et al. 2004).

${ }^{\mathrm{r}}$ From OGLE and MACHO data (McGowan et al. 2007; Schmidtke \& Cowlev 2007b).

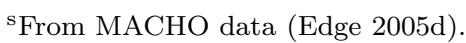

${ }^{\mathrm{t}}$ From MACHO data (Schmidtke \& Cowlev 2006).

${ }^{u}$ From OGLE data (Schmidtke et al. 2006).

${ }^{v}$ From OGLE data (Schmidtke \& Cowlev 2006).

${ }^{\mathrm{w}}$ From OGLE data (Schmidtke \& Cowlev 2005b).

${ }^{\mathrm{x}}$ From OGLE and MACHO data (Schmidtke et al. 2004). 
${ }^{\mathrm{y}}$ From OGLE and MACHO data (Edge et al. 2005c).

${ }^{\mathrm{z}}$ From OGLE data (McGowan et al. 2007).

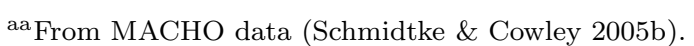

${ }^{\mathrm{ab}}$ From MACHO data (Cowley \& Schmidtke 2003; Schmidtke et al. 2004). 
We thank A. Cowley for pointing out some glaring omissions in the final manuscript and also the anonymous referee for many useful comments that made this paper clearer.

Facilities: RXTE (PCA).

\section{A. Determining signal significance}

Scargle (1982) proposes the normalisation of the periodogram to the variance, $\sigma_{n}^{2}$, of the signal-free data, where $\sigma_{n}$ is the standard deviation; as such, Gaussian noise will have a power of 14 . Furthermore, the probability function Prob associated with the periodogram will be exponentially distributed 5 , and it can be shown that the probability that a periodic signal with power of $Z$ is due to noise is

$$
\mathrm{FAP}=1-\left(1-e^{-Z}\right)^{M}
$$

which is the False Alarm Probability, with $M$ being the number of independent frequencies, which we (rather conservatively) define as

$$
M=2 \times n_{f} \times \Delta f \times \tau
$$

where $n_{f}$ is the number of scanned frequencies, $\Delta f$ is the frequency interval used when calculating the periodogram, and $\tau$ is the duration of the observation.

A more useful number may be the significance of a detection, or how sure we are that it is real; this is simply 1 - FAP, expressed as a percentage. This is the value that will be used throughout this paper to estimate the importance and believability of a signal, and is given by

$$
\operatorname{Sig}(\%)=100 \times\left(1-e^{-Z}\right)^{M}
$$

\footnotetext{
${ }^{4}$ It is clear that most of the data from observations made by $R X T E$ during the survey contain contributions from a number of sources in the field of view, and their variance should not be used in the calculations. However, after analysing a large number of observations, it was found that the average power within the calculated power spectra was essentially 1 (likely due to the low $\mathrm{S} / \mathrm{N}$ ), which justifies our use of the light curve variance (including all the pulsar signals) instead of the variance of the noise, which would have been difficult to obtain.

${ }^{5}$ It will be of the form $P r o b=e^{-Z}$, which is the probability of detecting a peak in the periodogram above a certain power, $Z$.
} 
In the Lomb-Scargle periodogram, the peak-to-trough amplitude $A$ of the modulation in the signal is related to the power $P_{L S}$ through

$$
A=4 \sqrt{\frac{P_{L S} \sigma_{n}^{2}}{N}}
$$

where $N$ is the number of data points (Scargle 1982).

If the signal detected has any harmonics, its total power will be divided between the individual harmonic peaks in the power spectrum. Using only the fundamental to estimate the amplitude of the signal's pulsations could then severely underestimate it if there were considerable power in any of the harmonics (which is often the case). If the amplitude of all the harmonics is known, it can be shown that the total amplitude of the signal will be given by

$$
A_{\text {total }}=\sqrt{\sum_{i} A_{i}^{2}}
$$

From the error in the angular frequency detected at a certain power in the Lomb-Scargle periodogram (Horne \& Baliunas 1986), we derive the error on the period as

$$
d P=\frac{3}{4}\left(\frac{P^{2} \sigma_{n}}{\sqrt{N} \tau A}\right)
$$

where $A$ is the Lomb-Scargle amplitude given by Eq. (A5), and $\sigma_{n}$ is the standard deviation of the noise, although the standard deviation of the actual data is used, as explained earlier.

Apart from the global significance of a detection, we define an additional quantity, the local significance, as the significance of a peak at frequency $f$, within a region of frequency space extending $5 \%$ of $f$ to either side of it.

\section{B. Luminosity and magnetic field estimation}

If all the matter accreting onto a neutron star is converted into energy, the luminosity that will result is simply the gravitational energy lost by the in-falling mass (Frank et al. 2002): 


$$
L_{\mathrm{x}}=\frac{G M \dot{M}}{R}
$$

where $\dot{M}$ is the mass transfer rate. Some manipulation and substitution can provide a more manageable expression:

$$
L_{\mathrm{x}_{37}}=8.4 \times 10^{9}\left(\frac{M_{n} \dot{M}}{R_{n}}\right)
$$

which will give us the luminosity in terms of $10^{37} \mathrm{erg} \mathrm{s}^{-1}$, and where $M_{n}$ is the mass of the neutron star in units of $M_{\odot}, \dot{M}$ is the mass accretion rate in units of $M_{\odot} \mathrm{yr}^{-1}$, and $R_{n}$ is the radius of the neutron star in $\mathrm{km}$.

The angular momentum of a neutron star is given by

$$
\mathcal{L}_{n}=\frac{2 \pi I_{n}}{P_{\mathrm{s}}}
$$

where $P_{\mathrm{s}}$ is the spin period, and the moment of inertia is given by

$$
I_{n}=\frac{2}{5} M_{n} R_{n}^{2}
$$

with $M_{n}$ and $R_{n}$ being the mass and radius of the neutron star in standard units.

The torque experienced by a neutron star spinning up or down is given by

$$
|\tau| \equiv\left|\frac{d \mathcal{L}_{n}}{d t}\right|=2 \pi I_{n} \frac{\dot{P}_{\mathrm{s}}}{P_{\mathrm{s}}^{2}}
$$

with $\dot{P}_{\mathrm{s}}$ being the rate of change of the spin period (Frank et al. 2002).

For an accreting pulsar undergoing steady spin up/down, the applied torque will depend on the mass accretion rate, $\dot{M}$, and the angular momentum of matter in the accretion disc at the magnetospheric radius, $r_{m}$. This torque is given by

$$
\tau=\dot{M} \sqrt{G M_{n} r_{m}}
$$


The maximum torque possible will occur when $r_{m}=r_{c o}$, where the corotation radius 6 is given by

$$
r_{c o}=\left(\frac{G M_{n} P_{\mathrm{s}}^{2}}{4 \pi^{2}}\right)^{\frac{1}{3}}
$$

Substituting this value in Eq. (B6) will provide the expression for the maximum torque possible:

$$
\tau_{\max }=\dot{M}\left[\frac{G^{2} M_{n}^{2} P_{\mathrm{s}}}{2 \pi}\right]^{\frac{1}{3}}
$$

Clearly, $|\tau| \leq \tau_{\max }$, so using Eqs. B5] and B8, and substituting the expression for the moment of inertia from Eq. (B4), we find the accretion rate will be

$$
\dot{M} \geq \frac{2}{5} R_{n}^{2} \dot{P}_{\mathrm{s}}\left[\frac{16 \pi^{4} M_{n}}{P_{\mathrm{s}}^{7} G^{2}}\right]^{\frac{1}{3}} \mathrm{~kg} \mathrm{~s}^{-1}
$$

substituting this value in Eq. (B1) we finally obtain the lower limit on the luminosity that will be produced through accretion:

$$
L_{\mathrm{x}_{37}} \geq \frac{2 R_{\mathrm{n}} \dot{P}_{\mathrm{s}}}{5 \times 10^{30}}\left[\frac{16 \pi^{4} G M_{n}^{4}}{P_{\mathrm{s}}^{7}}\right]^{\frac{1}{3}}
$$

which will be in units of $10^{37} \mathrm{erg} \mathrm{s}^{-1}$ if S.I. units are used (and the neutron star mass is in $\left.M_{\odot}\right)$. This equation will allow the estimation of the luminosity associated with an outburst if the average spin up/down is measured.

One further value that can be estimated is the magnetic field of the neutron star. Rearranging Eq. (6.24) of Frank et al. (2002), and using the period in place of the frequency, a constraint can be placed on its value:

$$
B_{12} \leq\left[3.4 \times 10^{-4} R_{n}^{-2} M_{n}^{\frac{-10}{7}} L_{\mathrm{x} 37}^{\frac{6}{7}} \frac{P_{\mathrm{s}}^{2}}{\dot{P}_{\mathrm{s}}}\right]^{\frac{-7}{2}}
$$

\footnotetext{
${ }^{6}$ The corotation radius is defined as the radius at which matter in the disc is moving at the same speed as the neutron star's surface.
} 
where the magnetic field will be in units of $10^{12} \mathrm{G}$ if $M_{n}$ is in $M_{\odot}$ and $R_{n}$ in metres. In the

present work we assume values for the neutron star's radius and mass of $R_{n}=10^{4} \mathrm{~m}$ and $M_{n}=1.4 M_{\odot}$, respectively.

\section{REFERENCES}

Ables J. G., Jacka C. E., Hall P. J., Hamilton P. A., McConnell D., McCulloch P. M., 1987, IAU Circ., 4422, 1

Bell J. F., 1994, Proceedings of the Astronomical Society of Australia, 11, 81

Blackburn J. K., 1995, in Shaw R. A., Payne H. E., Hayes J. J. E., eds, Astronomical Data Analysis Software and Systems IV Vol. 77 of Astronomical Society of the Pacific Conference Series, FTOOLS: A FITS Data Processing and Analysis Software Package. pp 367-+

Buckley D. A. H., Coe M. J., Stevens J. B., van der Heyden K., Angelini L., White N., Giommi P., 2001, Mon. Not. R. Astron. Soc., 320, 281

Carstairs I. R., 1992, Ph.D. Thesis, University of Southampton

Chakrabarty D., Levine A., Clark G., Takeshima T., Wilson C., Finger M., 1998, IAU Circ., 7048,1

Charles P. A., Southwell K. A., O’Donoghue D., 1996, IAU Circ., 6305, 2

Clark G., Doxsey R., Li F., Jernigan J. G., van Paradijs J., 1978, Astrophys. J., Lett., 221, L37

Clark G., Remillard R., Woo J., 1996, IAU Circ., 6282, 1

Clark G. W., Remillard R. A., Woo J. W., 1997, Astrophys. J., Lett., 474, L111+

Coe M. J., Edge W. R. T., Galache J. L., McBride V. A., 2005, Mon. Not. R. Astron. Soc., 356,502

Coe M. J., Haigh N. J., Laycock S. G. T., Negueruela I., Kaiser C. R., 2002, Mon. Not. R. Astron. Soc., 332, 473

Coe M. J., Stevens J. B., Buckley D. A. H., Charles P. A., Southwell K. A., 1998, Mon. Not. R. Astron. Soc., 293, 43 
Corbet R. H. D., 1984, Astron. Astrophys., 141, 91

Corbet R., Marshall F. E., Lochner J. C., Ozaki M., Ueda Y., 1998, IAU Circ., 6803, 1

Corbet R. H. D., Marshall F. E., Coe M. J., Laycock S., Handler G., 2001, Astrophys. J., Lett., 548, L41

Corbet R., Markwardt C. B., Marshall F. E., Laycock S., Coe M., 2002, IAU Circ., 7932, 2

Corbet R., Markwardt C. B., Marshall F. E., Coe M. J., Edge W. R. T., Laycock S., 2003a, IAU Circ., 8064, 4

Corbet R. H. D., Markwardt C. B., Marshall F. E., Coe M. J., Edge W. R. T., Laycock S., 2003b, The Astronomer's Telegram, 163, 1

Corbet R. H. D., Edge W. R. T., Laycock S., Coe M. J., Markwardt C. B., Marshall F. E., 2003c, AAS/High Energy Astrophysics Division, 7, 1

Corbet R. H. D., Laycock S., Marshall F. E., Markwardt C. B., Coe M. J., 2003d, The Astronomer's Telegram, 209, 1

Corbet R. H. D., Markwardt C. B., Coe M. J., Edge W. R. T., Laycock S., Marshall F. E., 2003e, The Astronomer's Telegram, 214, 1

Corbet R. H. D., Coe M. J., Edge W. R. T., Laycock S., Markwardt C. B., Marshall F. E., 2004a, The Astronomer's Telegram, 277, 1

Corbet R. H. D., Laycock S., Coe M. J., Marshall F. E., Markwardt C. B., 2004b, in AIP Conf. Proc. 714: X-ray Timing 2003: Rossi and Beyond Monitoring and Discovering X-ray Pulsars in the Small Magellanic Cloud. pp 337-341

Corbet R. H. D., Markwardt C. B., Coe M. J., Edge W. R. T., Laycock S., Marshall F. E., 2004c, The Astronomer's Telegram, 273, 1

Corbet R. H. D., Markwardt C. B., Marshall F. E., Coe M. J., Edge W. R. T., Galache J. L., Laycock S., 2004d, The Astronomer's Telegram, 347, 1

Cowley A. P., Schmidtke P. C., 2003, Astrophys. J., 126, 2949

Cowley A. P., Schmidtke P. C., 2004, Astrophys. J., 128, 709

Cowley A. P., Schmidtke P. C., McGrath T. K., Ponder A. L., Fertig M. R., Hutchings J. B., Crampton D., 1997, Publ. Astron. Soc. Pac., 109, 21 
de Jager O. C., Raubenheimer B. C., North A. R., Nel H. I., van Urk G., 1988, Astrophys. J., 329, 831

Edge W. R. T., Coe M. J., 2003, Mon. Not. R. Astron. Soc., 338, 428

Edge W. R. T., Coe M. J., Corbet R. H. D., Markwardt C. B., Laycock S., 2004a, The Astronomer's Telegram, 225, 1

Edge W. R. T., Coe M. J., Corbet R. H. D., Markwardt C. B., Laycock S., Marshall F. E., 2004b, The Astronomer's Telegram, 216, 1

Edge W. R. T., Coe M. J., Galache J. L., McBride V. A., Corbet R. H. D., Markwardt C. B., Laycock S., 2004c, Mon. Not. R. Astron. Soc., 353, 1286

Edge W. R. T., Coe M. J., McBride V. A., 2004d, The Astronomer's Telegram, 217, 1

Edge W. R. T., Coe M. J., Galache J. L., 2005a, The Astronomer's Telegram, 405, 1

Edge W. R. T., Coe M. J., Galache J. L., McBride V. A., Corbet R. H. D., Markwardt C. B., Laycock S., Marshall F. E., 2005b, The Astronomer's Telegram, 426, 1

Edge W. R. T., Coe M. J., Galache J. L., McBride V. A., Corbet R. H. D., Okazaki A. T., Laycock S., Markwardt C. B., Marshall F. E., Udalski A., 2005c, Mon. Not. R. Astron. Soc., 361, 743

Edge W. R. T., 2005d, Ph.D. Thesis, University of Southampton

Fabrycky D., 2005, Mon. Not. R. Astron. Soc., 359, 117

Filipović M. D., Haberl F., Pietsch W., Morgan D. H., 2000, Astron. Astrophys., 353, 129

Frank J., King A., Raine D. J., 2002, Accretion Power in Astrophysics: Third Edition. Cambridge University Press

Galache J. L., Corbet R. H. D., Coe M. J., Laycock S., Markwardt C. B., Marshall F. E., 2005, The Astronomer's Telegram, 674, 1

Grimm H.-J., Gilfanov M., Sunyaev R., 2003, Mon. Not. R. Astron. Soc., 339, 793

Haberl F., Filipović M. D., Pietsch W., Kahabka P., 2000, Astron. Astrophys. Suppl. Ser., 142,41

Haberl F., Pietsch W., 2004, Astron. Astrophys., 414, 667 
Haberl F., Pietsch W., Kahabka P., 2007, The Astronomer's Telegram, 1095, 1 Haberl F., Pietsch W., 2005, Astron. Astrophys., 438, 211

Haberl F., Pietsch W., Schartel N., Rodriguez P., Corbet R. H. D., 2004, The Astronomer's Telegram, 219, 1

Haberl F., Sasaki M., 2000, Astron. Astrophys., 359, 573

Horne J. H., Baliunas S. L., 1986, Astrophys. J., 302, 757

Hughes J. P., 1994, Astrophys. J., Lett., 427, L25

Imanishi K., Yokogawa J., Koyama K., 1998, IAU Circ., 7040, 2

Israel G. L., Campana S., Covino S., Dal Fiume D., Gaetz T. J., Mereghetti S., Oosterbroek T., Orlandini M., Parmar A. N., Ricci D., Stella L., 2000, Astrophys. J., Lett., 531, L131

Israel G. L., Stella L., Angelini L., White N. E., Giommi P., Covino S., 1997, Astrophys. J., Lett., 484, L141+

Israel G. L., Stella L., Campana S., Covino S., Ricci D., Oosterbroek T., 1998, IAU Circ., 6999, 1

Jahoda K., Markwardt C. B., Radeva Y., Rots A., Stark M. J., Swank J. H., Strohmayer T. E., Zhang W., 2005, ArXiv Astrophysics e-prints, astro-ph/0511531

Jahoda K., Swank J. H., Giles A. B., Stark M. J., Strohmayer T., Zhang W., Morgan E. H., 1996, in Proc. SPIE Vol. 2808, p. 59-70, EUV, X-Ray, and Gamma-Ray Instrumentation for Astronomy VII, Oswald H. Siegmund; Mark A. Gummin; Eds. In-orbit performance and calibration of the Rossi X-ray Timing Explorer (RXTE) Proportional Counter Array (PCA). pp 59-70

Kahabka P., Pietsch W., 1998, IAU Circ., 6840, 1

Kaspi V. M., Johnston S., Manchester R. N., Bailes M., Bell J. F., Bessell M., Lyne A. G., D'Amico N., 1993, Bulletin of the American Astronomical Society, 25, 1434

Lamb R. C., Fox D. W., Macomb D. J., Prince T. A., 2002, Astrophys. J., Lett., 574, L29

Lamb R. C., Macomb D. J., Prince T. A., Majid W. A., 2002, Astrophys. J., Lett., 567, L129

Lamb R. C., Prince T. A., Macomb D. J., Finger M. H., 1999, IAU Circ., 7081, 4 
Laycock S., Corbet R. H. D., Coe M. J., Marshall F. E., Markwardt C., Edge W., 2003, Mon. Not. R. Astron. Soc., 339, 435

Laycock S., Corbet R. H. D., Coe M. J., Marshall F. E., Markwardt C., Lochner J., 2005, Astrophys. J., Suppl. Ser., 161, 96

Laycock S., Corbet R. H. D., Perrodin D., Coe M. J., Marshall F. E., Markwardt C., 2002, Astron. Astrophys., 385, 464

Laycock S. G. T., 2002, Ph.D. Thesis, University of Southampton

Lochner J. C., Marshall F. E., Whitlock L. A., Brandt N., 1998, IAU Circ., 6814, 1

Lochner J. C., Whitlock L. A., Corbet R. H. D., Marshall F. E., 1999a, American Astronomical Society Meeting Abstracts, $194^{\text {th }}$ Meeting

Lochner J. C., Whitlock L. A., Corbet R. H. D., Marshall F. E., 1999b, Bulletin of the American Astronomical Society, 31, 742

Lomb N. R., 1976, Astrophys. Space. Sci., 39, 447

Macomb D. J., Finger M. H., Harmon B. A., Lamb R. C., Prince T. A., 1999, Astrophys. J., Lett., 518, L99

Macomb D. J., Fox D. W., Lamb R. C., Prince T. A., 2003, Astrophys. J., Lett., 584, L79

Maeder A., Grebel E. K., Mermilliod J.-C., 1999, Astron. Astrophys., 346, 459

Majid W. A., Lamb R. C., Macomb D. J., 2004, Astrophys. J., 609, 133

Marshall F. E., Lochner J. C., Santangelo A., Cusumano G., Israel G. L., dal Fiume D., Orlandini M., Frontera F., Parmar A. N., Corbet R. H. D., 1998, IAU Circ., 6818, 1

Marshall F. E., Lochner J. C., Takeshima T., 1997, IAU Circ., 6777, 2

McGowan K. E., Coe M. J., Schurch M., McBride V. A., Galache J. L., Edge W. R. T., Corbet R. H. D., Laycock S., Udalski U., Buckley D. A. H., 2007, to appear in Mon. Not. R. Astron. Soc.

Press W. H., Rybicki G. B., 1989, Astrophys. J., 338, 277

Sasaki M., Haberl F., Keller S., Pietsch W., 2001, Astron. Astrophys., 369, L29

Sasaki M., Pietsch W., Haberl F., 2003, Astron. Astrophys., 403, 901 
Scargle J. D., 1982, Astrophys. J., 263, 835

Schmidtke P. C., Cowley A. P., Levenson L., Sweet K., 2004, Astron. J., 127, 3388

Schmidtke P. C., Cowley A. P., 2005a, The Astronomer's Telegram, 648, 1

Schmidtke P. C., Cowley A. P., 2005b, Astron. J., 130, 2220

Schmidtke P. C., Cowley A. P., 2006, Astron. J., 132, 919

Schmidtke P. C., Cowley A. P., Udalski A., 2006, Astron. J., 132, 971

Schmidtke P. C., Cowley A. P., 2007, The Astronomer's Telegram, 1181, 1

Schmidtke P. C., Cowley A. P., 2007, American Astronomical Society Meeting Abstracts, 211, \#03.06

Schmidtke P. C., Cowley A. P., Udalski A., 2007, The Astronomer's Telegram, 1316, 1

Southwell K. A., Charles P. A., 1996, Mon. Not. R. Astron. Soc., 281, L63

Stanimirovic S., Staveley-Smith L., Dickey J. M., Sault R. J., Snowden S. L., 1999, Mon. Not. R. Astron. Soc., 302, 417

Stella L., White N. E., Rosner R., 1986, Astrophys. J., 308, 669

Stevens J. B., Coe M. J., Buckley D. A. H., 1999, Mon. Not. R. Astron. Soc., 309, 421

Torii K., Yokogawa J., Imanishi K., Koyama K., 2000, IAU Circ., 7428, 3

Tsujimoto M., Imanishi K., Yokogawa J., Koyama K., 1999, Publ. Astron. Soc. Jpn., 51, L21

Ueno M., Yamaguchi H., Takagi S.-I., Yokogawa J., Koyama K., 2004, Publ. Astron. Soc. Jpn., 56, 175

Wilson C., Finger M., 1998, IAU Circ., 7048, 1

Wilson C. A., Finger M. H., Coe M. J., Negueruela I., 2003, Astrophys. J., 584, 996

Yokogawa J., Imanishi K., Koyama K., Nishiuchi M., Mizuno N., 2002, Publ. Astron. Soc. Jpn., 54, 53

Yokogawa J., Imanishi K., Tsujimoto M., Koyama K., Nishiuchi M., 2003, Publ. Astron. Soc. Jpn., 55, 161 
Yokogawa J., Imanishi K., Ueno M., Koyama K., 2000, Publ. Astron. Soc. Jpn., 52, L73

Yokogawa J., Torii K., Imanishi K., Koyama K., 2000, Publ. Astron. Soc. Jpn., 52, L37

Yokogawa J., Koyama K., 1998a, IAU Circ., 6853, 2

Yokogawa J., Koyama K., 1998b, IAU Circ., 7009, 3

Yokogawa J., Koyama K., 1998c, IAU Circ., 7028, 1

Yokogawa J., Torii K., Kohmura T., Imanishi K., Koyama K., 2000, Publ. Astron. Soc. Jpn., $52, \mathrm{~L} 53$ 